\section{Addendum to the Canadian Guidelines for the Management of Plaque Psoriasis 2009}

\author{
Canadian Psoriasis Guidelines Addendum Committee
}

Journal of Cutaneous Medicine and Surgery 20I6, Vol. 20(5) 375-43I (C) The Author(s) 2016

Reprints and permissions: sagepub.com/journalsPermissions.nav DOI: $10.1177 / 1203475416655705$ jcms.sagepub.com

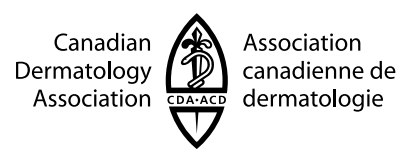

\title{
Notes to Readers
}

Disclaimer: As in the original guidelines, physicians should use their best clinical judgment when determining whether and how to apply treatment recommendations in the individualized care of patients. This document is not intended to replace the guidance found in the relevant Canadian product monographs or other official information available for the therapeutics discussed. Every reasonable effort has been made to ensure the accuracy of this document. Any errors made here will be corrected in the next edition of the guidelines.

Drug recall: Please disregard all previous recommendations for alefacept, as it was withdrawn from the market in $20 \mathrm{I}$.

Drug names: As in the original guidelines document, generic names have been used throughout this document. Any new trade name or generic name used in the addendum has been has listed in Appendix I at the end of this document.

Citation: Canadian Psoriasis Guidelines Addendum Committee. Addendum to the Canadian Guidelines for the Management of Plaque Psoriasis, May 2016.

Address academic correspondence to: Kim Alexander Papp, MD, PhD, FRCPC, Probity Medical Research, I35 Union Street East, Waterloo, Ontario, Canada N2J IC4. Email: kapapp@probitymedical.com

Directions for readers: This addendum should be used in conjunction with the original 2009 Canadian Guidelines for the Management of Plaque Psoriasis as a tool to guide physicians in clinical decision making. All changes to the content of the 2009 guidelines are presented by chapter, which correspond to the chapters in the original document. All new information is cross-referenced by page number and section/subsection to the original guidelines where the addendum applies. A table listing only new recommendations or modifications to existing recommendations follows each chapter.

Legend

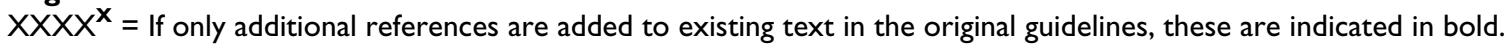

All original text from the 2009 Guidelines is underlined.

All new recommendations or modifications to existing recommendations are indicated in bold. 


\section{Table of Contents}

COMMITTEE, REVIEWERS, AND EDITORIAL SUPPORT

ACKNOWLEDGEMENTS.

CHAPTER I: INTRODUCTION.

CHAPTER 2: METHODS.

CHAPTER 3: DEFINITIONS.

CHAPTER 4: DELIVERY OF CARE..

CHAPTER 5: MANAGEMENT OF MILD PLAQUE PSORIASIS

CHAPTER 6: MANAGEMENT OF MODERATE TO SEVERE PLAQUE PSORIASIS

CHAPTER 7: SPECIAL POPULATIONS AND CIRCUMSTANCES.

CHAPTER 8: EXACERBATION AND FLARE OF PSORIASIS

CHAPTER 9: MANAGEMENT OF FACIAL, FLEXURAL, AND GENITAL PSORIASIS..

CHAPTER I0: MANAGEMENT OF NAIL PSORIASIS.

CHAPTER II: MANAGEMENT OF SCALP PSORIASIS.

CHAPTER I2: MANAGEMENT OF PALMOPLANTAR PSORIASIS. 


\section{Committee, Reviewers, and Editorial Support}

\section{Guidelines Committee}

Steering Committee

Kim Alexander Papp, MD, PhD, FRCPC (Chair)

K. Papp Clinical Research, Waterloo, Ontario

Probity Medical Research, Waterloo, Ontario

Wayne Gulliver, MD, FRCPC

Newlab Clinical Research Inc, St John's, Newfoundland

Memorial University, St John's, Newfoundland

Charles W. Lynde, MD, FRCPC

Lynderm Research Inc, Markham, Ontario

Probity Medical Research, Markham, Ontario

University of Toronto, Toronto, Ontario

Yves Poulin, MD, FRCPC

Centre Dermatologique du Québec, Métropolitain, Québec, Québec

Université Laval, Québec, Québec

Expert Panel

David N. Adam, MD, FRCPC

CCA Medical Research, Ajax, Ontario

Probity Medical Research, Ajax, Ontario

University of Toronto, Toronto, Ontario

Benjamin Barankin, MD, FRCPC

Toronto Dermatology Centre, Toronto, Ontario

Probity Medical Research, Toronto, Ontario

Kirk Barber, MD, FRCPC

Calgary Dermatology Treatment \& Research, Calgary, Alberta

University of Calgary, Calgary, Alberta

Marc Bourcier, MD, FRCPC

Dermatology Clinic, Moncton, New Brunswick

Faculty of Medicine, Sherbrooke University, Sherbrooke,

Quebec

Melinda Gooderham, MSc, MD, FRCPC

Skin Centre for Dermatology, Peterborough, Ontario

Probity Medical Research, Queen's University, Kingston,

Ontario

Lyn C. Guenther, MD, FRCPC

The Guenther Dermatology Research Centre, London, Ontario

The University of Western Ontario, London, Ontario

Vincent C. Ho, MD, FRCPC, FRCP (Lon)

The Skin Care Center, Vancouver, British Columbia

University of British Columbia, Vancouver, British Columbia
Andrei Metelitsa, MD, FRCPC, FAAD

Institute for Skin Advancement, Calgary, Alberta

Probity Medical Research, University of Calgary, Calgary,

Alberta

Neil H. Shear, MD, FRCPC, FACP

Sunnybrook Health Sciences Centre, Toronto, Ontario

University of Toronto, Toronto, Ontario

Ronald B. Vender, MD, FRCPC

Dermatrials Research, Hamilton, Ontario

McMaster University, Hamilton, Ontario

Norman Wasel, MD, FRCPC, FAAD

Stratica Medical, Edmonton, Alberta

Probity Medical Research, University of Alberta,

Edmonton, Alberta

Marni C. Wiseman, MD, FRCPC

SkinWise Dermatology Clinic, Winnipeg, Manitoba

Probity Medical Research, University of Manitoba,

Winnipeg, Manitoba

\section{Editorial Support}

Editorial support was provided by Kataka Medical Communication of Montreal, Quebec.

Rebecca Stuart, BSc

Shereen Joseph, BSc

Eva Chanda, MSc

Sylvanne Daniels, MSc, $\mathrm{PhD}$

Raeven Geist-Deschamps, DO(c)

Devon Phillips

David Rosenberg

\section{Community Reviewers}

A. David Burden, MD, FRCP

University of Glasgow, Scotland, UK

Prof. Paolo Gisondi, MD

University of Verona, Faculty of Medicine, Department of Dermatology and Venereology, Italy

Ricardo Romiti, MD

Department of Dermatology, Hospital das Clínicas University of São Paulo, (USP), Brazil

Fernando Valenzuela, MD

University of Chile, Santiago, Chile;

Probity Medical Research 


\section{Acknowledgements}

Financial assistance for the development of the Addendum to the Guidelines was generously provided by the following sponsors (in alphabetical order):

\section{Leaders:}

AbbVie Canada Inc

Amgen Canada Inc

LEO Pharma Inc

Novartis Canada

\section{Partners:}

Pfizer Canada

Sponsors were permitted to submit unpublished manuscripts for consideration by the Addendum Guidelines Committee, with the proviso that the article had to be accepted for peerreviewed publication by a designated cut-off date. Sponsors were not involved in any other aspect of the Guidelines' development, nor were they informed of the makeup of the Addendum Guidelines Committee.

\section{Disclosure of Potential Conflicts of Interest}

The following committee members report that during the 5 years prior to the development of the Addendum to the Guidelines, they had conflicts of interest linked to the following companies:

David N. Adam: Consultancy*: AbbVie Canada Inc; Actelion Pharmaceuticals Canada Inc; Amgen Canada Inc; Celgene Corp; Eli Lilly Canada Inc; Galderma Canada Inc; JanssenOrtho Inc; LEO Pharma Inc; Merck Frosst Canada Inc; Novartis Pharmaceuticals Canada Inc; Pfizer Canada Inc; Sanofi-Aventis Canada Inc; Stiefel Laboratories Inc; and Valeant Canada Inc. Contract research: Amgen Canada Inc; Celgene Corp; Coherus Biosciences Inc; Eli Lilly Canada Inc; Galderma Canada Inc; Janssen-Ortho Inc; LEO Pharma Inc; and Pfizer Canada Inc.

Benjamin Barankin: Consultancy*: AbbVie Canada Inc; Actelion Pharmaceuticals Canada Inc; Amgen Canada Inc; Galderma Canada Inc; Janssen-Ortho Inc; Johnson \& Johnson Services Inc; LEO Pharma Inc; Novartis Pharmaceuticals Canada Inc; and Valeant Canada Inc. Contract research: AbbVie Canada Inc; Amgen Canada Inc; Galderma Canada Inc; LEO Pharma Inc; Novartis Pharmaceuticals Canada Inc; and Pfizer Canada Inc.

Kirk Barber: Consultancy*/Contract research: AbbVie Canada Inc; Amgen Canada Inc; Boehringer Ingelheim (Canada) Ltd; Celgene Corp; Eli Lilly Canada Inc;
Janssen-Ortho Inc; LEO Pharma Inc; Merck Serono SA; Novartis Pharmaceuticals Canada Inc; Valeant; and Pfizer Canada.

Marc Bourcier: Consultancy*: Abbott Laboratories Ltd; Amgen Canada Inc; Astellas Pharma Canada Inc; Barrier Therapeutics Inc; EMD Serono Canada Inc; Graceway Pharmaceuticals; Janssen-Ortho Inc; LEO Pharma Inc; Merck Frosst Canada Ltd; Novartis Pharmaceuticals Canada Inc; Pfizer Canada Inc; Schering-Plough Canada Inc; Stiefel Laboratories Inc; and Wyeth. Contract research: Abbott Laboratories Ltd; Amgen Canada Inc; Astellas Pharma Canada Inc; Barrier Therapeutics Inc; EMD Serono Canada Inc; LEO Pharma Inc; Janssen-Ortho Inc; Novartis Pharmaceuticals Canada Inc; Pfizer Canada Inc; ScheringPlough Canada Inc; and Wyeth.

Melinda Gooderham: Consultancy*: AbbVie Canada Inc; Amgen Canada Inc; Astellas Pharma Canada Inc; Celgene Corp; Eli Lilly Canada Inc; Galderma Canada Inc; JanssenOrtho Inc; Kyowa Hakko Kirin Pharma Inc; LEO Pharma Inc; Novartis Pharmaceuticals Canada Inc; and Pfizer Canada Inc. Contract research: AbbVie Canada Inc; Amgen Canada Inc; Astellas Pharma Canada Inc; Celgene Corp; Dermira Canada Inc; Eli Lilly Canada Inc; Galderma Canada Inc; Janssen-Ortho Inc; Kyowa Hakko Kirin Pharma Inc; LEO Pharma Inc; Merck Frosst Canada Inc; Novartis Pharmaceuticals Canada Inc; Pfizer Canada Inc; Takeda Canada Inc; and Xoma.

Wayne Gulliver: Consultancy*: Abbott Laboratories Ltd; Actelion Pharmaceuticals Canada Inc; Amgen Canada Inc; Astellas Pharma Canada Inc; Galderma Canada Inc; JanssenOrtho Inc; LEO Pharma Inc; Merck Serono SA; Stiefel Laboratories Inc; and Valeant Canada Inc.

Lyn C. Guenther: Consultancy*: AbbVie Canada Inc; Amgen Canada Inc; Celgene Corp; Eli Lilly Canada Inc; Galderma Canada Inc; Janssen-Ortho Inc; LEO Pharma Inc; Merck Frosst Canada Ltd; Novartis Pharmaceuticals; Pfizer Canada Inc; and Tribute Pharmaceuticals Inc. Contract research: AbbVie Canada Inc; Amgen Canada Inc; Boehringer Ingelheim (Canada) Ltd; Celgene Corp; Eli Lilly Canada Inc; Galderma Canada Inc; Janssen-Ortho Inc; LEO Pharma Inc; Merck Frosst Canada Ltd; Novartis Pharmaceuticals; and Pfizer Canada Inc.

Vincent Ho: Consultancy*: AbbVie Canada Inc; Amgen Canada Inc; Janssen-Ortho Inc; Novartis Pharmaceuticals Canada Inc; LEO Pharma Inc. Contract research: AbbVie Canada Inc; Amgen Canada Inc; Janssen-Ortho Inc; Novartis Pharmaceuticals Canada Inc; Regeneron; and Pfizer Canada Inc.

Charles W. Lynde: Consultancy*/Contract research: AbbVie Canada Inc; Amgen Canada Inc; Boehringer 
Ingelheim (Canada) Ltd; Celgene Corp; Eli Lilly Canada Inc; Janssen-Ortho Inc; LEO Pharma Inc; Merck Serono SA; Novartis Pharmaceuticals Canada Inc; and Pfizer Canada Inc.

Andrei Metelitsa: Consultancy*: AbbVie Canada Inc; Amgen Canada Inc; Celgene Corp; Galderma Canada Inc; LEO Pharma Inc; and Novartis Pharmaceuticals Canada Inc.

Kim A. Papp: Consultancy*: Abbott Laboratories Ltd; Amgen Canada Inc; Astellas Pharma Canada Inc; Barrier Therapeutics Inc; Baxter; Boehringer-Ingelheim; Bristol Myers Squibb Canada Co; Celgene Corp; Dermira Canada Inc; Eli Lilly Canada Inc; Galderma Canada Inc; Genentech; Graceway Pharmaceuticals; GSK; Isotechnika Inc; JanssenOrtho Inc; Kyowa Hakko Kirin Pharma Inc; LEO Pharma Inc; Merck (MSD); Merck-Serono SA; Novartis Pharmaceuticals Canada Inc; Pfizer Canada Inc; ScheringPlough Canada Inc; Stiefel Laboratories Inc; Takeda; and Xoma. Contract research: Abbott Laboratories Ltd; Amgen Canada Inc; Astellas Pharma Canada Inc; Barrier Therapeutics Inc; Baxter; Boehringer-Ingelheim; Bristol Myers Squibb Canada Co; Celgene Corp; Dermira Canada Inc; Galderma; Janssen-Ortho Inc; LEO Pharma Inc; Merck (MSD); Novartis Pharmaceuticals Canada Inc; Pfizer Canada Inc; Regeneron; Takeda; and USB.

Yves Poulin: Consultancy*: AbbVie Canada Inc; Amgen Canada Inc; Celgene Corp; Eli Lilly Canada Inc; Stiefel Laboratories Inc; and Janssen-Ortho Inc. Contract research: AbbVie Canada Inc; Amgen Canada Inc; Boehringer Ingelheim (Canada) Ltd; Bristol-Myers Squibb Canada Co; Celgene Corp; Centocor Ortho Biotech Inc; Eli Lilly Canada Inc; Galderma Canada Inc; Incyte Corp; LEO Pharma Inc; Merck Frosst Canada Inc; Novartis Pharmaceuticals Canada Inc; Pfizer Canada Inc; and Takeda Canada Inc.
Neil Shear: Consultancy*: AbbVie Canada Inc; Amgen Canada Inc; Celgene Corp; Janssen-Ortho Inc; LEO Pharma Inc; Eli Lilly Canada Inc; Novartis Pharmaceuticals Canada Inc; Roche Canada Inc; and Xoma Ltd.

Ronald B. Vender: Consultancy*: AbbVie Canada Inc; Amgen Canada Inc; Celgene Corp; Centocor Ortho Biotech Inc; Galderma Canada Inc; Janssen-Ortho Inc; Johnson \& Johnson Services Inc; LEO Pharma Inc; Eli Lilly Canada Inc; Novartis Pharmaceuticals Canada Inc; Pfizer Canada Inc; and Valeant Canada Inc. Contract research: AbbVie Canada Inc; Amgen Canada Inc; Celgene Corp; Centocor Ortho Biotech Inc; Galderma Canada Inc; Janssen-Ortho Inc; Johnson \& Johnson Services Inc; LEO Pharma Inc; Eli Lilly Canada Inc; Novartis Pharmaceuticals Canada Inc; Pfizer Canada Inc; Takeda Canada Inc; and Valeant Canada Inc.

Norman Wasel: Consultancy*: Abbott Laboratories Ltd; Amgen Canada Inc; Astellas Pharma Canada Inc; Biogen Idec Canada Inc; EMD Serono Canada Inc; Isotechnika Inc; Janssen-Ortho Inc; Ortho Biotech; Schering-Plough Canada Inc; and Wyeth. Contract research: Abbott Laboratories Ltd; Amgen Canada Inc; Astellas Pharma Canada Inc; Biogen Idec Canada Inc; Celgene Corp; Centocor Ortho Biotech Inc; Eli Lilly Canada Inc; EMD Serono Canada Inc; Isotechnika Inc; LEO Pharma Inc; Merck Frosst Canada Inc; Novartis Pharmaceuticals Canada Inc; Pfizer Canada Inc; Takeda Canada Inc and Wyeth.

Marni C. Wiseman: Consultancy*: AbbVie Canada Inc; Amgen Canada Inc; Janssen-Ortho Inc; LEO Pharma Inc; and Novartis Pharmaceuticals Canada Inc.

*Consultancy includes advisory boards, editorial advice, podium presentations, and travel grants. 


\section{List of Abbreviations}

\author{
ACCEPT $=$ Active Comparator $($ CNTO1275/Enbrel $)$ \\ Psoriasis Trial \\ $\mathrm{ACE}=$ angiotensin converting-enzyme \\ $\mathrm{ADA}=$ antidrug antibody \\ $\mathrm{ARBs}=$ angiotensin II receptor blockers \\ $\mathrm{BIW}=$ biweekly \\ $\mathrm{BSA}=$ body surface area \\ $\mathrm{CCT}=$ crude coal tar \\ $\mathrm{CDLQI}=$ Children's Dermatology Life Quality Index \\ $\mathrm{CKD}=$ chronic kidney disease \\ CoPSI $=$ Copenhagen Psoriasis Severity Index \\ $\mathrm{CVD}=$ cardiovascular disease \\ DLQI = Dermatology Life Quality Index
}

ERASURE = Efficacy of response and safety of two fixed secukinumab regimens in psoriasis

ESTEEM $=$ Efficacy and safety trial evaluating the effects of apremilast in psoriasis 1 and 2

FIXTURE $=$ Full year investigative examination of secukinumab vs. etanercept using two dosing regimens to determine efficacy in psoriasis

$\mathrm{HBV}=$ hepatitis $\mathrm{B}$ virus

$\mathrm{HPCH}=$ hydroxypropyl chitosan

$\mathrm{HR}=$ hazard ratio

$\mathrm{HRQoL}=$ health-related quality of life

IGA = Investigator's Global Assessment

$\mathrm{IL}=$ interleukin

$\mathrm{JAK}=$ Janus kinase

JAK-STAT $=$ Janus kinase signal transducer and activator of transcription

$\mathrm{LCD}=$ liquor carbonis distillate

$\mathrm{LoE}=$ level of evidence

$\mathrm{MI}=$ myocardial infarction

NAPSI $=$ Nail Psoriasis Severity Index

$\mathrm{NB}=$ narrowband

NSAID $=$ nonsteroidal anti-inflammatory drug
ODS $=$ overall disease severity score

$\mathrm{OPT}=$ Oral treatment psoriasis rrial

$\mathrm{OR}=$ odds ratio

PASI $=$ Psoriasis Area and Severity Index

$\mathrm{PDL}=$ pulse dye laser

$\mathrm{PEDF}=$ pigment epithelium-derived factor

PGA $=$ Physician's Global Assessment

$\mathrm{PKC}=$ protein kinase $\mathrm{C}$

PPP $=$ palmoplantar pustular psoriasis

PsA $=$ psoriatic arthritis

PSI $=$ Psoriasis Symptom Inventory

PSOLAR $=$ Psoriasis Longitudinal Assessment and Registry

PSSI $=$ Psoriasis Scalp Severity Index

PUVA $=$ UVA with psoralen

$\mathrm{QoL}=$ quality of life

$\mathrm{RCT}=$ randomized controlled trial

REFINE $=$ A randomized, blinded assessor study to evaluate the efficacy and safety of etanercept $50 \mathrm{mg}$ once weekly plus as needed topical agent vs. etanercept $50 \mathrm{mg}$ twice weekly in patients with moderate to severe plaque psoriasis

ReNB-UVB $=$ retinoid + NB-UVB

RePUVA $=$ retinoid + PUVA

REVEAL $=$ Randomized controlled evaluation of adalimumab every other week dosing in moderate to severe psoriasis trial

SEBs $=$ subsequent entry biologics

SLQI $=$ Scalp Life Quality Index

sPGA $=$ static $\mathrm{PGA}$

$\mathrm{TCI}=$ topical calcineurin inhibitor

TPS $=$ Target Plaque Severity

$\mathrm{TNF}=$ tumor necrosis factor

UNITE $=$ Utilization of narrow-band ultraviolet light B therapy and etanercept for the treatment of psoriasis

VDS $=$ vitamin D analogues plus topical steroids

VEGF $=$ vascular endothelial growth factor 


\section{Chapter I: Introduction}

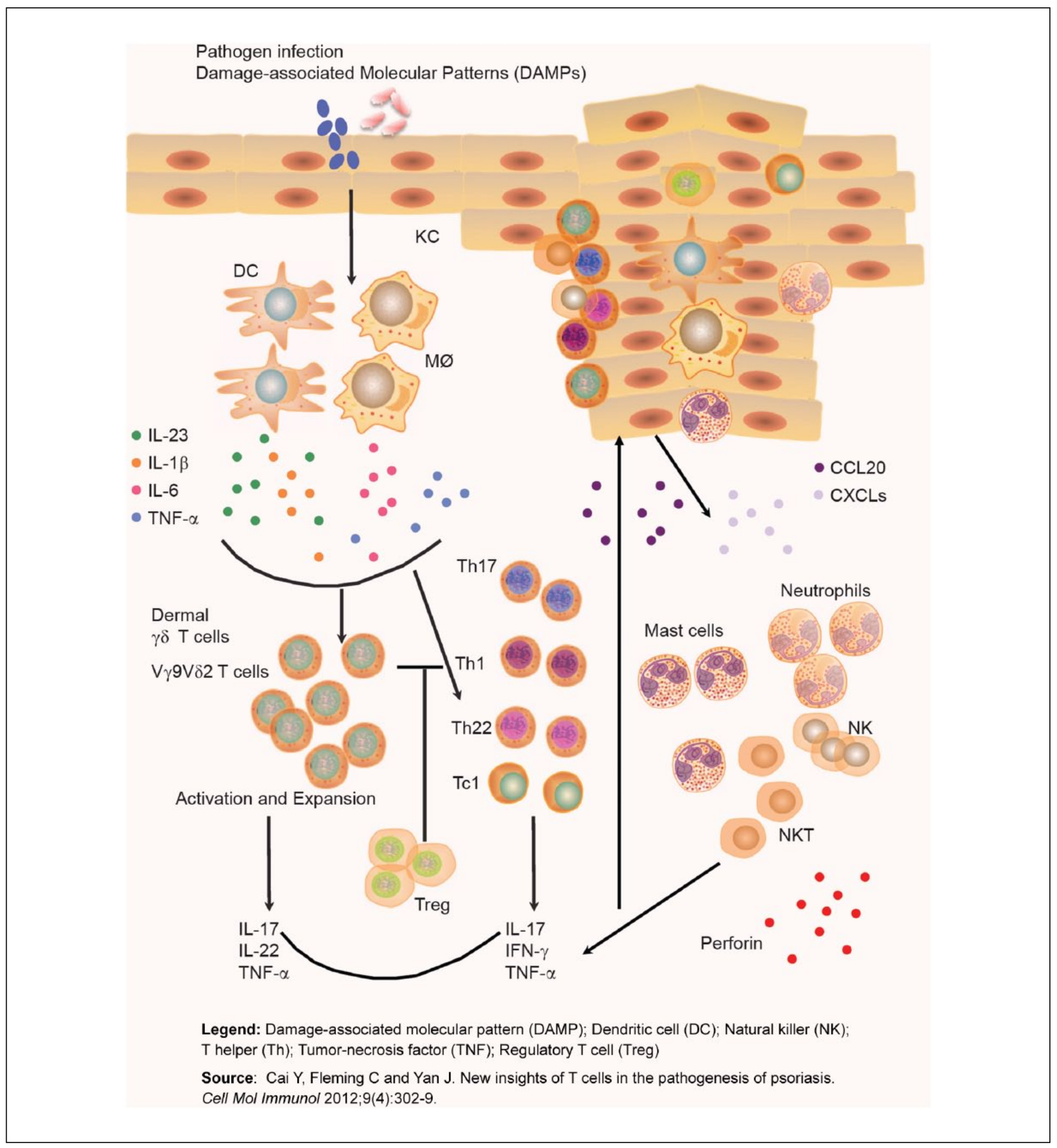

Figure I. Proposed psoriasis immunopathogenesis. 
Addition to section on page 2

\section{The Central Role of T Cells in Psoriasis Pathophysiology}

Since the publication of the 2009 Canadian Guidelines for the Management of Plaque Psoriasis, significant advances have been made in determining the role of T cells in inflammatory and autoimmune diseases. We now understand that Th1, Th17, Treg, and Th22 cells interact with each other. Whereas psoriasis was previously thought to be a Th1mediated disease, Th17 cells are now seen to play a pivotal role. ${ }^{1}$ Interleukin (IL)-17-producing dermal $\gamma \delta \mathrm{T}$ cells probably play a vital role in pathogenesis. It has been proposed that psoriasis is first triggered by foreign antigens that activate dendritic cells and macrophages. The activated, professional antigen-presenting cells release IL-23 and IL-1 $\beta$ along with other proinflammatory cytokines. Proinflammatory cytokines in turn activate dermal $\gamma \delta \mathrm{T}$ cells as well as other IL-17-producing cells. The host of activated cells copiously produce IL-17, which further promotes the immune response. IL-17 (primarily), IL-22, and tumor necrosis factor $\alpha$ (TNF$\alpha)$ then induce a keratinocyte hyperproliferation. A hyperproliferative response leads to the release of chemokines. Released chemokines attract more immune effector cells into the skin. Immune effector cells (ie, neutrophils, mast cells, natural killer cells, and natural killer $\mathrm{T}$ cells) contribute to the proinflammatory response, resulting in escalated production of cytokines and chemokines. This amplified positive feedback loop leads to the development of psoriatic plaques (see Figure 1).

Thus, unsurprisingly, newer biologic agents that target IL-12/23 or IL-17 have shown efficacy in psoriasis treatment. Also in development are orally administered small molecules that target the Th17 pathway. ${ }^{2}$ See Chapter 15 and Chapter 17 in this addendum for a review of these emerging biologics.

Addition to section on page 3

\section{Psoriasis Epidemiology and Natural History}

Two recent surveys of Canadian patients report that the burden of this disease is substantial in patients with moderate to severe psoriasis. ${ }^{3,4}$

\section{References}

1. Cai Y, Fleming C, Yan J. New insights of T cells in the pathogenesis of psoriasis. Cell Mol Immunol. 2012;9(4):302-309.

2. Elloso MM, Gomez-Angelats M, Fourie AM. Targeting the Th17 pathway in psoriasis. J Leukoc Biol. 2012;92(6):11871197.

3. Papp K, Valenzuela F, Poulin Y, et al. Epidemiology of moderate-to-severe plaque psoriasis in a Canadian surveyed population. J Cutan Med Surg. 2010;14(4):167-174.

4. Lynde CW, Poulin Y, Guenther L, et al. The burden of psoriasis in Canada: insights from the pSoriasis Knowledge IN Canada (SKIN) survey. J Cutan Med Surg. 2009;13(5):235-252. 


\section{Chapter 2: Methods}

The Steering Committee developed a list of specific clinical questions to guide the literature search (see Appendix II). The clinical questions allowed for the generation of key terms that were used by a professional librarian to search PubMed, MEDLINE, CINAHL, Cochrane (including the Controlled Trials Register), Scopus, Web of Science, and EMBASE for papers on psoriasis and antipsoriatic therapies published from 2007 or later. All peer-reviewed literature was considered. Papers were also identified by checking the references lists of reviews and other guidelines, hand-searching personal libraries, forward-tracking citation, and identifying further key literature (including newly published papers) as writing proceeded.

In all, 800 peer-reviewed research articles were identified and the citations subsequently maintained in an EndNote library. Non-randomly controlled research as well as research not in the relevant population was excluded.
Meeting abstracts and posters, narrative reviews, and commentaries were excluded (except as a source of references) because they could not be appraised. Case reports were excluded from the initial literature search although writers were permitted to cite them if necessary to address clinical questions that could not be answered by systematic reviews.

Sponsors were invited to submit peer-reviewed articles and unpublished manuscripts for consideration until a cut-off date of October 1, 2013. Unpublished data included in the initial draft of the update were required to be accepted for publication by January 31, 2014, to remain part of the final document.

Finally, Chapter 17 summarizes pertinent papers in the literature on plaque psoriasis published from January 2014 to December 2015. The Guidelines Committee was invited to submit all papers that they deemed essential to include in this update for the purpose of this chapter. 


\section{Chapter 3: Definitions}

Addition to Table 1 on page 12

Table I. Metrics Used for Defining Disease Severity.

\begin{tabular}{|c|c|}
\hline Measure & Description \\
\hline $\begin{array}{l}\text { Copenhagen Psoriasis Severity } \\
\text { Index (CoPSI) }\end{array}$ & $\begin{array}{l}\text { An index that comprises the assessment of } 3 \text { signs: erythema, plaque thickness, and scaling, each on } \\
\text { a } 4 \text {-point scale ( } 0 \text {, none; I, mild; } 2 \text {, moderate; } 3 \text {, severe), at each of } 10 \text { sites: face, scalp, upper limbs } \\
\text { (excluding hands and wrists), hands and wrists, chest and abdomen, back, buttocks and sacral area, } \\
\text { genitalia, lower limbs (excluding feet and ankles), and feet and ankles. }\end{array}$ \\
\hline $\begin{array}{l}\text { Children's Dermatology Life } \\
\text { Quality Index (CDLQI) }\end{array}$ & $\begin{array}{l}\text { Dermatology Life Quality Index (DLQI) modified for use in children. It comprises } 10 \text { questions and, like the } \\
\text { adult version, assesses } 6 \text { domains of health-related quality of life (HRQoL): symptoms and feelings, leisure, } \\
\text { school or holidays, personal relationships, sleep, and treatment. Each question has } 4 \text { possible responses, } \\
\text { scored from } 0 \text { to 3. Possible total scores range from } 0 \text { (no impairment) to } 30 \text { (maximum impairment). }\end{array}$ \\
\hline Scalp Life Quality Index (SLQI) ${ }^{4}$ & DLQI modified for the scalp. \\
\hline
\end{tabular}

\section{References}

1. Berth-Jones J, Thomson J, Papp K. A study examining interrater and intrarater reliability of a novel instrument for assessment of psoriasis: the Copenhagen Psoriasis Severity Index. $\mathrm{Br}$ J Dermatol. 2008;159(2):407-412.

2. Lewis-Jones MS, Finlay AY. The Children's Dermatology Life Quality Index (CDLQI): initial validation and practical use. Br J Dermatol. 1995;132:942-949.
3. Holme SA, Man I, Sharpe JL, et al. The Children's Dermatology Life Quality Index: validation of the cartoon version. $\mathrm{Br} J$ Dermatol. 2003;148:285-290.

4. Mrowietz U, Macheleidt O, Eicke C, et al. Effective treatment and improvement of quality of life in patients with scalp psoriasis by topical use of calcipotriol/betamethasone (Xamiol(R)-gel): results. $J$ Dtsch Dermatol Ges. 2011;9(10):825-831. 


\section{Chapter 4: Delivery of Care}

\section{Addition to Chapter 4 introductory section on page 16}

The Psoriasis Longitudinal Assessment and Registry (PSOLAR) study is a disease-based registry that, as of 2011, had enrolled patients from 266 centres globally, 35 of which are in Canada (179 in the United States, 49 in Europe, and 3 in Mexico). ${ }^{1}$ This international registry can tell us more about the psoriasis patient population. PSOLAR enrolled 9495 patients between June 2007 and August 2011. Just over half of the PSOLAR population is male $(54.5 \%)$. At time of entry, the mean age of patients was 48.8 years, and patients had been affected by the disease for a mean of 17.4 years. Even with therapy, the mean percentage of body surface area affected was $12.3 \%$ upon entry while the mean body surface area affected at historical peak disease activity was $29.5 \%$. The majority of patients had multiple comorbidities at entry $(38.8 \%$ cardiovascular disease [CVD], 37.1\% psoriatic arthritis [PsA], 12.8\% diabetes).

In 2007 , an online survey was conducted among psoriasis patients in Canada. ${ }^{2}$ This Canadian-specific survey captured information from 514 patients living with psoriasis. The study was conducted to assess the severity and burden of disease on patients. Most respondents had moderate to severe disease, yet the study reported that only $18 \%$ of respondents used systemic medication and/or phototherapy. Within this group, $25 \%$ of patients had $\geq 3 \%$ body surface area involvement, and $39 \%$ had $\geq 10 \%$ involvement. Although most patients reported moderate to severe disease, $92 \%$ of patients were using topical prescription medications and $84 \%$ were using over-the-counter medications. This is consistent with results from a US National Psoriasis Foundation survey, which recorded that $18 \%$ of respondents had severe psoriasis and $87 \%$ used topical or overthe-counter medications. The study surmised that patients with moderate to severe psoriasis may not be receiving optimal care or treatment appropriate for their level of severity. ${ }^{2}$
Addition to subsection on page 16

\section{The Locus of Psoriasis Care}

\section{Outpatient Care for Severe Psoriasis}

The PSOLAR study is a disease-based registry that could help physicians improve care for outpatients with severe psoriasis. Of the 9495 patients enrolled, 7476 had been exposed to at least 1 biologic agent, and 2019 had received treatments such as conventional systemic agents, phototherapy, and topical treatments. The registry can help physicians assess the risk and benefit of therapy in the outpatient psoriasis population, especially those with severe disease. The international registry is intended to serve as a way to identify safety concerns among psoriasis patients, particularly those treated with biologic agents.

Addition to section on page 17

\section{Treatment Adherence and Physician Engagement}

A 2012 review of studies on treatment adherence in psoriasis found that up to $95 \%$ of patients underdose when using topical medications. ${ }^{3}$ The review reported the mean compliance level to be between $50 \%$ and $60 \%$ among patients. ${ }^{3}$

\section{References}

1. Papp KA, Strober B, Augustin M, et al. PSOLAR: design, utility, and preliminary results of a prospective, international, disease-based registry of patients with psoriasis who are receiving, or are candidates for, conventional systemic treatments or biologic agents. J Drugs Dermatol. 2012;11(10):1210-1217.

2. Wasel N, Poulin Y, Andrew R, et al. A Canadian self-administered online survey to evaluate the impact of moderate-to-severe psoriasis among patients. J Cutan Med Surg. 2009;13(6):294-302.

3. Cribier B. Adherence of local (and general) treatments in psoriasis. Ann Dermatol Venereol. 2012;139(suppl 1):S13-S17. 


\section{Chapter 5: Management of Mild Plaque Psoriasis}

Addition to section on page 19

\section{Corticosteroids}

Corticosteroids are the most widely used agents for the topical treatment of psoriasis and have been the mainstay of therapy for over half a century. They are well tolerated and often efficacious, and they come in a variety of forms, including ointments, creams, gels, lotions, sprays, foams, and solutions.

Addition to section on page 20

\section{Vitamin $D_{3}$ Analogues}

A 2012 study of 48 patients using topical vitamin $\mathrm{D}_{3}$ highlights the issue of adherence as a determinant of treatment outcomes. ${ }^{1}$ Patients receiving tacalcitol or maxacalcitol for more than a month without sufficient improvement were switched to calcipotriol ointment, and vice versa (patients initially on calcipotriol were switched to tacalcitol or maxacalcitol). This intervention of rotating from one topical vitamin $\mathrm{D}_{3}$ agent to another with similar efficacy led to improved clinical outcomes. The proposed explanation is that the rotation triggers an increase in patient adherence. Thus, vitamin $\mathrm{D}_{3}$ rotation therapy may be a way to improve adherence in patients with low motivation in long-term care. Furthermore, researchers speculate that what was thought to be tachyphylaxis with topical steroid use could instead be a manifestation of patient frustration with continued application of the same agent.

Calcitriol is the naturally occurring active form of vitamin $\mathrm{D}_{3}$. It has long been used topically to treat plaque psoriasis in Europe and is now available in Canada. In a 2009 review of short-term and long-term clinical research on the use of topical calcitriol $3 \mu \mathrm{g} / \mathrm{g}$ monotherapy, this ointment was found to be effective, safe, and well tolerated. ${ }^{2}$ In 2 large randomized, double-blind clinical trials, application of this ointment twice daily for 8 weeks showed clearing or minimal residual psoriasis in more than a third of patients treated. ${ }^{3}$ Use of this topical therapy also resulted in improved signs and symptoms of plaque elevation, erythema, scaling, and pruritus. ${ }^{4}$ The study found that calcitriol exhibited a low risk of adverse events even after a year of use with no significant difference from vehicle. Use of this ointment has not been shown to induce sensitization, phototoxicity, or photosensitization.
Systemic absorption of calcitriol was found to be minimal and did not alter systemic calcium homeostasis significantly. It can be used as monotherapy or in combination regimens to treat psoriasis.

\section{Addition to section on page 20}

\section{Retinoids}

A small comparison study of right-to-left bilateral symmetrical lesions found that the efficacy of tazarotene $0.1 \%$ gel was also comparable to that of calcipotriol $0.005 \%$ ointment. ${ }^{5}$ A double-blind, randomized, right-left comparison study of bilateral symmetrical lesions found that topical tazarotene $0.1 \%$ was less effective than clobetasol propionate $0.05 \%$ cream. ${ }^{6}$ Tazarotene was found to be more effective for induration than erythema and scaling of psoriatic lesions.

\section{Addition to section on page 20}

\section{Anthralin and Tars}

A nonblinded study comparing the efficacy and tolerability of crude coal tar (CCT) 5\% ointment with that of topical tazarotene $0.1 \%$ gel found that CCT had a clinical efficacy comparable to tazarotene gel. In this right-left bilateral symmetrical comparison, no side effects were reported for patients receiving CCT. ${ }^{7}$

Coal tar reformulated as topical liquor carbonis distillate (LCD) $15 \%$ solution was shown to be cosmetically acceptable, well tolerated, ${ }^{8}$ and effective compared with calcipotriol $0.005 \%$ cream. $^{9}$

\section{Addition to section on page 21}

\section{Combination Therapy}

A 2011 systematic literature review and meta-analysis compared the efficacy of monotherapy using vitamin D analogues with that of combination therapy with vitamin D analogues plus topical steroids (VDS). ${ }^{10}$ The probability of success was found to be 2-fold higher for VDS compared with VD alone.

Calcipotriol/betamethasone is now available in Canada in a gel formulation. A randomized controlled study in 458 patients found this gel combination $(50 \mu \mathrm{g} / \mathrm{g}$ calcipotriol and $0.5 \mathrm{mg} / \mathrm{g}$ 
betamethasone) to be more effective than tacalcitol ointment $(4 \mu \mathrm{g} / \mathrm{g})$ or the gel vehicle alone. ${ }^{4}$ Over an 8 -week treatment period, the 183 patients using the 2-compound gel exhibited a faster response and lower withdrawal rate due to adverse reactions when compared with patients in the other 2 treatment arms. The researchers suggested that the gel formula offers patients a more cosmetically acceptable alternative, which may boost patient compliance.

An open-label, multicentre study found that combination treatment with calcitriol $3 \mu \mathrm{g} / \mathrm{g}$ twice daily on weekdays and clobetasol propionate spray $0.05 \%$ twice daily on weekends was effective, was well-tolerated, and showed high patient satisfaction. ${ }^{11}$ In 4 weeks, $80 \%$ of the 70 patients receiving this weekday-weekend therapy went from an overall disease severity score of "moderate" to "clear," "almost clear," or "mild." In addition, a small randomized open-label trial in 30 patients found that daily treatment with calcipotriol and tacrolimus in combination (calcipotriol in the morning and tacrolimus in the evening) was as effective and possibly better tolerated than calcipotriol monotherapy (twice daily) and more effective than tacrolimus alone (twice daily). ${ }^{12}$

Addition to section on page 22

\section{Other Approaches}

\section{Nonmedicinal Topical Treatments}

An open-label trial demonstrated that emollient use following therapy with topical corticosteroids can delay the time to disease flare. $^{13}$

Topical zinc pyrithione has long been used in the treatment of psoriasis, and most evidence supporting the efficacy of this treatment has been anecdotal.

A double-blind, randomized, placebo-controlled trial was conducted to assess the safety and efficacy of XP-828L, which is a protein found in whey. ${ }^{14}$ Patients who were given $5 \mathrm{~g} / \mathrm{d}$ for 56 days scored better on the Physician's Global Assessment (PGA) than those receiving placebo. Although the results were statistically significant, actual disease improvement was clinically irrelevant.

Addition to section on page 22

\section{Measure of Success}

Adherence to topical treatments by patients tends to be much poorer than reported in clinical studies. ${ }^{15}$

\section{Recommendations (page 23)}

All new recommendations or modifications to existing recommendations are indicated in bold.

\begin{tabular}{lc}
\hline Recommendation and Level of Evidence & Grade of Recommendation \\
\hline $\begin{array}{l}\text { Other appropriate first-line options include topical calcipotriol (Refs. I, 2, I6, LoE I++) and calcipotriol/ } \\
\text { betamethasone dipropionate in combination (Refs. 4, I0, 69, LoE I++). }\end{array}$ & Grade A \\
$\begin{array}{l}\text { Topical calcitriol can be used as first-line monotherapy or in combination regimens for } \\
\text { patients with mild plaque psoriasis (Refs. 2, 3, LoE I++). }\end{array}$ & Grade A \\
$\begin{array}{l}\text { For appropriate patients, tazarotene may be used, either alone (Ref. 6, LoE 2-) or in combination with } \\
\text { topical corticosteroids (Refs. 42, 49, LoE I+). }\end{array}$ & Grade B \\
$\begin{array}{l}\text { Coal tar reformulated as topical LCD I5\% may be used in appropriate patients (Ref. 9, LoE } \\
\text { I+, Ref. 8, LoE 2+). }\end{array}$ & Grade B
\end{tabular}

\section{References}

1. Imafuku S, Kubota Y, Ito K, et al. Effects of rotation of topical vitamin D3 in chronic plaque-type psoriasis. J Dermatol. 2012;39(3):275-277.

2. Kircik L. Efficacy and safety of topical calcitriol 3 microg/g ointment, a new topical therapy for chronic plaque psoriasis. J Drugs Dermatol. 2009;8(8 suppl):S9-S16.

3. Lebwohl M, Menter A, Weiss J, et al. Calcitriol 3 microg/g ointment in the management of mild to moderate plaque type psoriasis: results from 2 placebo-controlled, multicenter, randomized double-blind, clinical studies. J Drugs Dermatol. 2007;6(4):428-435.

4. Langley RG, Gupta A, Papp K, et al. Calcipotriol plus betamethasone dipropionate gel compared with tacalcitol ointment and the gel vehicle alone in patients with psoriasis vulgaris: a randomized, controlled clinical trial. Dermatology. 2011;222(2):148-156.

5. Kaur I, Dogra S, Jain R, et al. Comparative study of calcipotriol $(0.005 \%)$ ointment and tazarotene $(0.05 \%$ and $0.1 \%)$ gel in the treatment of stable plaque psoriasis. Indian J Dermatol Venereol Leprol. 2008;74(5):471-474.

6. Angelo JS, Kar BR, Thomas J. Comparison of clinical efficacy of topical tazarotene $0.1 \%$ cream with topical clobetasol propionate $0.05 \%$ cream in chronic plaque psoriasis: a double-blind, randomized, right-left comparison study. Indian J Dermatol Venereol Leprol. 2007;73(1):65.

7. Kumar U, Kaur I, Dogra S, et al. Topical tazarotene vs. coal tar in stable plaque psoriasis. Clin Exp Dermatol. 2010;35(5): 482-486. 
8. Brouda I, Edison B, Van Cott A, et al. Tolerability and cosmetic acceptability of liquor carbonis distillate (coal tar) solution $15 \%$ as topical therapy for plaque psoriasis. Cutis. 2010;85(4):214-220.

9. Alora-Palli MB, Perkins AC, Van Cott A, et al. Efficacy and tolerability of a cosmetically acceptable coal tar solution in the treatment of moderate plaque psoriasis: a controlled comparison with calcipotriene (calcipotriol) cream. Am J Clin Dermatol. 2010;11(4):275-283.

10. Devaux S, Castela A, Archier E, et al. Topical vitamin D analogues alone or in association with topical steroids for psoriasis: a systematic review. J Eur Acad Dermatol Venereol. 2012;26(suppl 3):52-60.

11. Hudson CP, Kempers S, Menter A, et al. An open-label, multicenter study of the efficacy and safety of a weekday/weekend treatment regimen with calcitriol ointment 3 micro/g and clobetasol propionate spray $0.05 \%$ in the management of plaque psoriasis. Cutis. 2011;88(4):201-207.

12. Tirado-Sanchez A, Ponce-Olivera RM. Preliminary study of the efficacy and tolerability of combination therapy with calcipotriene ointment $0.005 \%$ and tacrolimus ointment $0.1 \%$ in the treatment of stable plaque psoriasis. Cutis. 2012;90(3):140-144.

13. Seite S, Khemis A, Rougier A, et al. Emollient for maintenance therapy after topical corticotherapy in mild psoriasis. Exp Dermatol. 2009;18(12):1076-1078.

14. Poulin Y, Bissonnette R, Juneau C, et al. XP-8281 in the treatment of mild to moderate psoriasis: randomized, double-blind, placebo-controlled study. J Cutan Med Surg. 2006;10(5): 241-248.

15. Devaux S, Castela A, Archier E, et al. Adherence to topical treatment in psoriasis: a systematic literature review. J Eur Acad Dermatol Venereol. 2012; 26(suppl 3):61-67. 


\section{Chapter 6: Management of Moderate to Severe Plaque Psoriasis}

Addition to subsections on pages 28-30

\section{Safety, Efficacy, and Tolerability of the Various Therapeutic Options}

\section{Systemic Therapy With Traditional and Biologic Agents}

A systematic review of the randomized controlled trial (RCT) data available before 2008 on systemic agents found that although all systemic agents provide benefits for the treatment of psoriasis, over the short term (12 weeks), infliximab and adalimumab are superior to other agents in terms of efficacy. ${ }^{1}$ This review was conducted before ustekinumab was approved for psoriasis.

\section{Systemic Agents}

\section{Acitretin}

A 2012 randomized, double-blind, parallel group study evaluated the efficacy of acitretin monotherapy at fixed doses of 25 , 35 , and $50 \mathrm{mg} / \mathrm{d}$ in 3 separate groups of patients with severe psoriasis (61 patients total). ${ }^{2}$ The study found that acitretin was effective across all 3 groups. Over a 12-week period, the mean Psoriasis Area and Severity Index (PASI) score of all patients decreased by $62.37 \%$ from baseline, with the acitretin dose of 35 $\mathrm{mg} / \mathrm{d}$ representing the optimum dosage in terms of efficacy and safety. A small study found that the addition of this retinoid may provide additional benefit when combined with biologic agents. ${ }^{3}$

\section{Cyclosporine}

A systematic review to evaluate the effect of cyclosporine on blood pressure from trials from 1980 to 2008 found that cyclosporine use is associated with a dose-related increase in blood pressure. ${ }^{4}$

An open-label, randomized, controlled study found that the addition of calcipotriol/betamethasone to low-dose cyclosporine improves clinical response in patients with moderate to severe plaque psoriasis. ${ }^{5}$

\section{Methotrexate}

Methotrexate is an inhibitor of folate biosynthesis and therefore impairs DNA replication. It was originally used in psoriasis for its cytostatic properties, but it is now recognized to be directly anti-inflammatory because of its effects on T cell gene expression patterns. ${ }^{25}$ Some but not all of these effects are related to folate depletion, consistent with clinical evidence that folate supplementation can diminish the efficacy of methotrexate treatment in psoriasis. . $^{2,6}$

Although the use of folate remains controversial in dermatological practice, $\underline{28}$ there appears to be little doubt that it improves the tolerability of methotrexate treatment and may therefore increase treatment adherence. ${ }^{29}$ Hence, this practice may be justified by greater real-world efficacy even if folate partially inhibits the therapeutic action of methotrexate. .6,30,6 $^{26}$

In terms of dosing, a randomized, double-blind, doseranging study found that $25 \mathrm{mg}$ of methotrexate once weekly was a more effective dose compared with $10 \mathrm{mg}$ weekly over 12 weeks in patients with severe psoriasis (51 total). ${ }^{7}$ A small $\mathrm{RCT}$ assessed the efficacy of sulfasalazine and pentoxifylline for the treatment of psoriasis. ${ }^{8}$ The study found that these drugs alone or in combination were safe but not more effective than methotrexate.

Addition to subsections on pages 30-32

\section{Biologic Agents Targeting TNF- $\alpha$}

\section{Adalimumab}

An open-label extension of this study lasting more than 3 years found that uninterrupted treatment with adalimumab maintained good long-term efficacy. ${ }^{9}$

Pooled data of placebo-controlled clinical trials of adalimumab also show this biologic agent to be effective and safe (low incidence of drug-associated adverse events). ${ }^{10,11}$

A study evaluating the use of adalimumab for chronic plaque psoriasis of the hands and feet found this agent to be well tolerated and efficacious over a 28 -week period. ${ }^{12}$

When used concurrently, calcipotriol/betamethasone may initially increase the effectiveness of adalimumab but does not add benefit to therapy after the first 4 weeks. ${ }^{13}$

An analysis performed in 2011 of the long-term safety data for adalimumab found that this biologic agent is associated with a relatively low incidence of adverse events over 3 years of treatment. ${ }^{14}$

For patients who need to interrupt therapy, adalimumab effectively recaptures response when introduced after partial relapse in patients who show high response rates prior to the interruption of therapy. ${ }^{15}$ In patients who have an inadequate response to other psoriasis treatments 


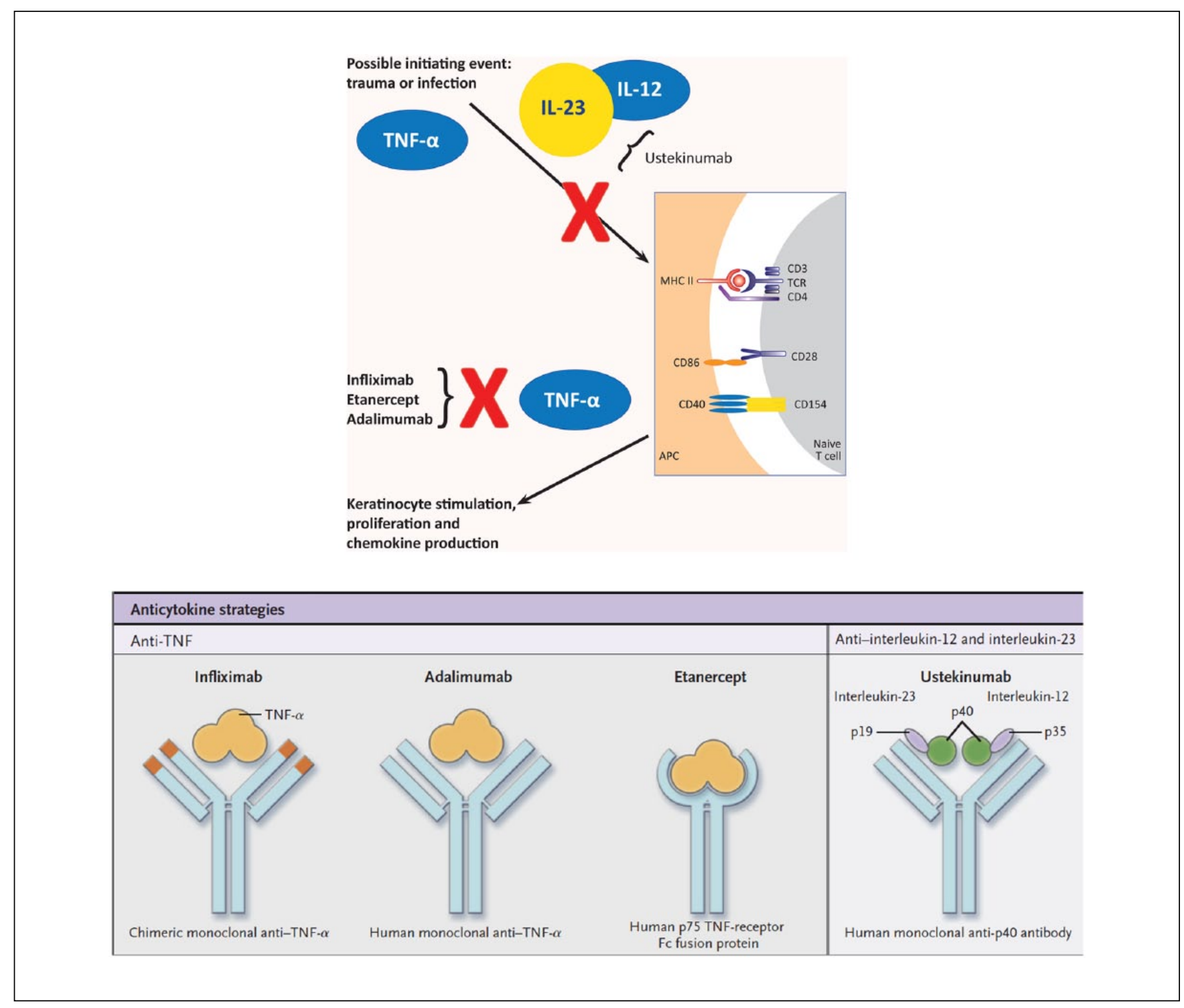

Figure I. Mechanism of action for biologic agents targeting TNF- $\alpha$ and IL-I2/23.

Biologic therapies that target TNF- $\alpha$ and IL-I2/23 are shown. Anticytokine therapies include infliximab, adalimumab, etanercept (anti-TNFs), and a monoclonal antibody against IL-12 and IL-23 (ustekinumab). Anti-TNF strategies have 3 variants: a humanized chimeric anti-TNF- $\alpha$ monoclonal antibody, a fully human monoclonal anti-TNF- $\alpha$ antibody, and a human p75 TNF-receptor Fc fusion protein. Finally, blocking of IL-I 2 and IL-23 is achieved by means of antibodies targeting the common p 40 chain of these cytokines. Essentially, ustekinumab targets differentiation cytokines for ThI and ThI7, blunting inflammation and preventing abnormal keratinocyte differentiation and proliferation.

Sources: Data from Garber K. Psoriasis: from bed to bench and back. Nat Biotechnol. 20I I;29:563-566; FDA Dermatological and Opthalmic Drugs Advisory Committee meeting, January 17, 2008; and Nestle FO, Kaplan DH, Barker J. Psoriasis. N Engl J Med. 2009;36I (5):496-509.

(including systemic therapy), adalimumab presents an effective alternative. ${ }^{16,17}$ PASI 75 responses after an interruption of therapy of up to 19 weeks ranged from moderate to excellent and were comparable to efficacy achieved with more than 3 years of continuous adalimumab therapy. ${ }^{18}$ Likewise, patients with an inadequate response to etanercept may respond to adalimumab. ${ }^{19}$

\section{Etanercept}

In patients who have an inadequate response to methotrexate, the addition of etanercept could help improve response. ${ }^{20} \mathrm{~A}$ study that analyzed integrated safety data from previous trials on etanercept found that the use of etanercept was associated with relatively few drug-associated adverse events in the 
long-term treatment of psoriasis. ${ }^{21}$ A study investigating the efficacy of etanercept $50 \mathrm{mg}$ biweekly compared with once weekly in patients with both psoriasis and PsA found that 50 mg twice weekly was better for treating skin symptoms, while $50 \mathrm{mg}$ weekly worked adequately in the treatment of affected joints and tendons. ${ }^{22}$ Continuous therapy with etanercept 50 $\mathrm{mg}$ biweekly is superior to intermittent therapy with this agent. $^{22}$

\section{Infliximab}

A small 2008 study also found that infliximab combined with methotrexate or azathioprine is effective as maintenance therapy in patients with moderate to severe psoriasis. ${ }^{23}$.

See Figure 1 for the mechanisms of action for biologic agents targeting TNF- $\alpha$ and IL-12/23.

New section to add on page 32

\section{Biologic Agents Targeting IL- I 2/23}

\section{Ustekinumab}

Ustekinumab gained approval for use in Canada at the end of 2008. Since the publication of the 2009 Canadian Guidelines for the Management of Plaque Psoriasis, this biologic agent has demonstrated long-term efficacy and safety. ${ }^{24-26}$ In a large patient population treated with this agent for up to 5 years, ustekinumab exhibited a low incidence of adverse events. ${ }^{24}$

Ustekinumab is approved for use in moderate to severe plaque psoriasis. The recommended dose is $45 \mathrm{mg}$ ustekinumab administered subcutaneously at weeks 0 and 4 and then every 12 weeks thereafter for patients weighing $100 \mathrm{~kg}$ or less. The recommended dose for patients weighing more than $100 \mathrm{~kg}$ is $90 \mathrm{mg}$, which should also be administered at weeks 0 and 4 and every 12 weeks thereafter. ${ }^{27}$ In cases where a loss of response was detected in patients, an improved response was seen when the dosage was increased from $45 \mathrm{mg}$ ustekinumab every 12 weeks to $90 \mathrm{mg}$ every 8 weeks. $^{26}$

Adverse events reported over 5 years were comparable with those reported for other biologic agents. ${ }^{28}$ No cumulative or dose-related toxicity was observed.

Refer to Figure 1 to review the targets of ustekinumab.

Addition to subsections on page 33 and 34

\section{Biologic Agents Targeting T Cells}

\section{Phototherapy and Photochemotherapy}

UVB monotherapy. An increase in treatments to 5 times weekly is not more effective than thrice-weekly treatments with narrowband (NB)-UVB. ${ }^{29}$ In a randomized, doubleblind, comparison study, high-dose UVB ( $40 \%$ increment) therapy resulted in better long-term efficacy in fewer treatments compared with low-dose therapy $\left(20 \%\right.$ increment) ${ }^{30}$ Patients were treated thrice weekly for 3 months. However, an adjustment in protocol was necessary 48 hours after treatment in the second week with high-dose UVB due to the increased incidence of erythema.

Unlike PUVA, it has not been established whether UVB is carcinogenic in humans ${ }^{92,93}$; however, speculation based on nonclinical data suggests that NB-UVB could be more carcinogenic than natural exposure to sunlight. ${ }^{81}$ There are no immediate prospects of UVB trials with sufficient power to quantify this risk ${ }^{81,31}$; in the absence of such evidence, it is prudent to use appropriate combination therapies when possible to reduce exposure to NB-UVB radiation.

UV combination regimens. A study evaluating the long-term efficacy of NB-UVB combined with topical calcitriol found the treatment to be safe and effective for up to 12 months of therapy. ${ }^{32}$ Treatment with petrolatum or CCT could improve the therapeutic benefit of NB-UVB. ${ }^{33}$

\section{Recommendations (pages 34-4I)}

Table I. Therapeutic Options for Ameliorating Moderate to Severe Plaque Psoriasis.

\begin{tabular}{llcr}
\hline Biologic Agents & \multicolumn{1}{c}{ Considerations } & Evidence for Efficacy as Monotherapy & References $^{\mathrm{a}}$ \\
\hline Ustekinumab & $\begin{array}{l}\text { Human monoclonal antibody targeting IL-I2 and } \\
\text { IL-23. } \\
\text { Approved for use in moderate to severe plaque } \\
\text { psoriasis. }\end{array}$ & LoE I+ & Refs. 25, 26, 28 \\
&
\end{tabular}

${ }^{a}$ Efficacy reflects at least a $75 \%$ improvement in PASI score, as determined by a statistically significant difference from placebo in studies of moderate to severe plaque psoriasis. 
Table 2. Therapeutic Regimens to be Considered for Potential Clearance of Moderate to Severe Plaque Psoriasis.

\begin{tabular}{|c|c|c|c|c|}
\hline Regimen & $\begin{array}{c}\text { Evidence for Disease } \\
\text { Clearance or Near Clearance } \\
\text { Within Approximately } 3 \\
\text { Months of Therapy }\end{array}$ & $\begin{array}{c}\text { Evidence for Disease } \\
\text { Clearance or Near Clearance } \\
\text { at Approximately } 6 \text { Months of } \\
\text { Therapy }\end{array}$ & $\begin{array}{c}\text { Evidence for Disease Clearance or } \\
\text { Near Clearance Beyond I Year of } \\
\text { Therapy }\end{array}$ & Notes \\
\hline Adalimumab & $\begin{array}{l}\text { Some patients may achieve } \\
100 \% \text { PASI reduction within } \\
16 \text { weeks of treatment. } \\
\left(\text { LoE } I++^{50}\right)\end{array}$ & $\begin{array}{l}\text { Some additional patients } \\
\text { achieve/maintain } 100 \% \text { PASI } \\
\text { reduction by } 24 \text { weeks of } \\
\text { treatment. (LoE } 2+++^{50} \text { ) }\end{array}$ & $\begin{array}{l}\text { Some patients may achievel } \\
\text { maintain } \geq 90 \% \text { PASI reduction } \\
\text { through at least } 160 \text { weeks of } \\
\text { treatment. (LoE } 2+^{9} \text { ) }\end{array}$ & $\begin{array}{l}\text { Intended for } \\
\text { ongoing } \\
\text { continuous } \\
\text { treatment }\end{array}$ \\
\hline Ustekinumab & $\begin{array}{l}\text { Some patients may } \\
\text { achieve } \geq 90 \% \text { PASI } \\
\text { reduction by week } 12 \text { of } \\
\text { treatment. (LoE } \mid+{ }^{24-26} \text { ) }\end{array}$ & $\begin{array}{l}\text { Some patients may achieve/ } \\
\text { maintain } \geq 90 \% \text { PASI } \\
\text { reduction by week } 28 \text { of } \\
\text { treatment. (LoE } 1+{ }^{24-26} \text { ) }\end{array}$ & $\begin{array}{l}\text { Some patients may maintain } \\
\geq 90 \% \text { PASI reduction } \\
\text { through at least } 244 \text { weeks of } \\
\text { treatment. (LoE } \mathrm{I}^{24} \text { ) }\end{array}$ & $\begin{array}{l}\text { Intended for } \\
\text { ongoing } \\
\text { continuous } \\
\text { therapy. }\end{array}$ \\
\hline
\end{tabular}

\section{References}

1. Schmitt J, Zhang Z, Wozel G, et al. Efficacy and tolerability of biologic and nonbiologic systemic treatments for moderate-tosevere psoriasis: meta-analysis of randomized controlled trials. Br J Dermatol. 2008;159(3):513-526.

2. Dogra S, Jain A, Kanwar AJ. Efficacy and safety of acitretin in three fixed doses of 25, 35 and $50 \mathrm{mg}$ in adult patients with severe plaque type psoriasis: a randomized, double blind, parallel group, dose ranging study. J Eur Acad Dermatol Venereol. 2012;27:305-311.

3. Smith EC, Riddle C, Menter MA, et al. Combining systemic retinoids with biologic agents for moderate to severe psoriasis. Int J Dermatol. 2008;47(5):514-518.

4. Robert N, Wong GWK, Wright JM. Effect of cyclosporine on blood pressure. Cochrane Database Syst Rev. 2010;(1):CD007893.

5. Vena GA, Galluccio A, Pezza M, et al. Combined treatment with low-dose cyclosporine and calcipotriol/betamethasone dipropionate ointment for moderate-to-severe plaque psoriasis: a randomized controlled open-label study. J Dermatolog Treat. 2012;23(4):255-260.

6. Chladek J, Simkova M, Vaneckova J, et al. The effect of folic acid supplementation on the pharmacokinetics and pharmacodynamics of oral methotrexate during the remission-induction period of treatment for moderate-to-severe plaque psoriasis. Eur J Clin Pharmacol. 2008;64(4):347-355.

7. Dogra S, Krishna V, Kanwar AJ, et al. Efficacy and safety of systemic methotrexate in two fixed doses of $10 \mathrm{mg}$ or $25 \mathrm{mg}$ orally once weekly in adult patients with severe plaque-type psoriasis: a prospective, randomized, double-blind, dose-ranging study. Clin Exp Dermatol. 2012;37(7):729-734.

8. el-Mofty M, el-Darouti M, Rasheed H, et al. Sulfasalazine and pentoxifylline in psoriasis: a possible safe alternative. J Dermatolog Treat. 2011;22(1):31-37.

9. Gordon KB, Papp KA, Poulin Y, et al. Long-term efficacy and safety of adalimumab in patients with moderate to severe psoriasis treated continuously over 3 years: results from an openlabel extension study for patients from REVEAL. J Am Acad Dermatol. 2012;66(2):241-251.

10. Papp K, Okun M, Vender R. Adalimumab in the treatment of psoriasis: pooled efficacy and safety results from three pivotal studies. J Cutan Med Surg. 2009;13(suppl 2):S58-S66.
11. Papp KA, Signorovitch J, Ramakrishnan K, et al. Effects of adalimumab versus placebo on risk of symptom worsening in psoriasis and subsequent impacts on health-related quality-oflife: analysis of pooled data from two randomized, doubleblind, placebo-controlled, multicentre clinical trials. Clin Drug Investig. 2011;31(1):51-60.

12. Leonardi C, Langley RG, Papp K, et al. Adalimumab for treatment of moderate to severe chronic plaque psoriasis of the hands and feet: efficacy and safety results from REACH, a randomized, placebo-controlled, double-blind trial. Arch Dermatol. 2011;147(4):429-436.

13. Thaci D, Ortonne JP, Chimenti S, et al. A phase IIIb, multicentre, randomized, double-blind, vehicle-controlled study of the efficacy and safety of adalimumab with and without calcipotriol/betamethasone topical treatment in patients with moderate to severe psoriasis: the BELIEVE study. $\mathrm{Br} J$ Dermatol. 2010:163(2):402-411.

14. Leonardi C, Papp K, Strober B, et al. The long-term safety of adalimumab treatment in moderate to severe psoriasis: a comprehensive analysis of all adalimumab exposure in all clinical trials. Am J Clin Dermatol. 2011;12(5):321-337.

15. Papp K, Crowley J, Ortonne JP, et al. Adalimumab for moderate to severe chronic plaque psoriasis: efficacy and safety of retreatment and disease recurrence following withdrawal from therapy. Br J Dermatol. 2011;164(2):434-441.

16. Papp K, Ho V, Teixeira HD, et al. Efficacy and safety of adalimumab when added to inadequate therapy for the treatment of psoriasis: results of PRIDE, an open-label, multicentre, phase IIIb study. J Eur Acad Dermatol Venereol. 2012;26(8):10071013.

17. Strober BE, Sobell JM, Duffin KC, et al. Sleep quality and other patient-reported outcomes improve after patients with psoriasis with suboptimal response to other systemic therapies are switched to adalimumab: results from PROGRESS, an open-label phase IIIB trial. Br J Dermatol. 2012;167(6):13741381.

18. Papp K, Menter A, Poulin Y, et al. Long-term outcomes of interruption and retreatment vs. continuous therapy with adalimumab for psoriasis: subanalysis of REVEAL and the open-label extension study. J Eur Acad Dermatol Venereol. 2013;27(5):634-642.

19. Strober BE, Poulin Y, Kerdel FA, et al. Switching to adalimumab for psoriasis patients with a suboptimal response to etanercept, methotrexate, or phototherapy: efficacy and 
safety results from an open-label study. J Am Acad Dermatol. 2011;64(4):671-681.

20. Zachariae C, Mork NJ, Reunala T, et al. The combination of etanercept and methotrexate increases the effectiveness of treatment in active psoriasis despite inadequate effect of methotrexate therapy. Acta Derm Venereol. 2008;88(5):495-501.

21. Pariser DM, Leonardi CL, Gordon K, et al. Integrated safety analysis: short- and long-term safety profiles of etanercept in patients with psoriasis. J Am Acad Dermatol. 2012;67(2):245-256.

22. Sterry W, Ortonne JP, Kirkham B, et al. Comparison of two etanercept regimens for treatment of psoriasis and psoriatic arthritis: PRESTA randomised double blind multicentre trial. BMJ. 2010;340:300.

23. Dalaker M, Bonesronning JH. Long-term maintenance treatment of moderate-to-severe plaque psoriasis with infliximab in combination with methotrexate or azathioprine in a retrospective cohort. J Eur Acad Dermatol Venereol. 2009;23(3):277282.

24. Kimball AB, Papp KA, Wasfi Y, et al. Long-term efficacy of ustekinumab in patients with moderate-to-severe psoriasis treated for up to 5 years in the PHOENIX 1 study. J Eur Acad Dermatol Venereol. 2013;27(12):1535-1545.

25. Leonardi CL, Kimball AB, Papp KA, et al. Efficacy and safety of ustekinumab, a human interleukin-12/23 monoclonal antibody, in patients with psoriasis: 76-week results from a randomised, double-blind, placebo-controlled trial (PHOENIX 1). Lancet. 2008;371:1665-1674.

26. Papp KA, Langley RG, Lebwohl M, et al. Efficacy and safety of ustekinumab, a human interleukin-12/23 monoclonal antibody, in patients with psoriasis: 52-week results from a randomised, double-blind, placebo-controlled trial (PHOENIX 2). Lancet. 2008;371:1675-1684.

27. Hsu S, Papp KA, Lebwohl MG, et al. Consensus guidelines for the management of plaque psoriasis. Arch Dermatol. 2012;148(1):95-102.

28. Papp KA, Griffiths CE, Gordon K, et al. Long-term safety of ustekinumab in patients with moderate-to-severe psoriasis: final results from 5 years of follow-up. $\mathrm{Br} J$ Dermatol. 2013;168(4):844-854.

29. Hallaji Z, Barzegari M, Balighi K, et al. A comparison of three times vs. five times weekly narrowband ultraviolet B phototherapy for the treatment of chronic plaque psoriasis. Photodermatol Photoimmunol Photomed. 2010;26(1): 10-15.

30. Kleinpenning MM, Smits T, Boezeman J, et al. Narrowband ultraviolet B therapy in psoriasis: randomized double-blind comparison of high-dose and low-dose irradiation regimens. Br J Dermatol. 2009;161(6):1351-1356.

31. Archier E, Devaux S, Castela E, et al. Carcinogenic risks of psoralen UV-A therapy and narrowband UV-B therapy in chronic plaque psoriasis: a systematic literature review. $J$ Eur Acad Dermatol Venereol. 2012;26(suppl 3):22-31.

32. Karakawa M, Komine M, Takekoshi T, et al. Duration of remission period of narrowband ultraviolet $\mathrm{B}$ therapy on psoriasis vulgaris. J Dermatol. 2011;38(7):655-660.

33. Abdallah MA, El-Khateeb EA, Abdel-Rahman SH. The influence of psoriatic plaques pretreatment with crude coal tar vs. petrolatum on the efficacy of narrow-band ultraviolet B: a half-vs.-half intraindividual double-blinded comparative study. Photodermatol Photoimmunol Photomed. 2011;27(5):226-230. 


\section{Chapter 7: Special Populations and Circumstances}

\section{Addition to section and subsections on page 46 and 47}

\section{Psoriasis in Children}

Recent studies show that psoriasis in children is associated with cardiovascular risk factors, including overweight, obesity, hyperlipidemia, hypertension, high blood glucose, diabetes, and metabolic syndrome. ${ }^{1-4}$ The odds of obesity and excess central adiposity (waist circumference) are higher in children with severe rather than mild psoriasis and are higher in childhood psoriasis than in adults. ${ }^{5}$ A large historical cohort study found that in females, overweight in adolescence increased the risk of moderate to severe psoriasis later on, suggesting that obesity precedes the onset of psoriasis in this group. ${ }^{6}$ Children with psoriasis and their caregivers should be apprised of the association between psoriasis and cardiovascular risk factors and should be encouraged to maintain a healthy lifestyle and weight. ${ }^{4}$

A 2010 systematic review of treatment efficacy and safety in children with psoriasis recommended calcipotriol as firstline treatment, with topical steroids if necessary. For treatment-resistant or moderate to severe psoriasis, dithranol (anthralin) was suggested, followed by short-term UVB in adolescents, with methotrexate as the systemic treatment of choice. Etanercept was recommended as a third-line option. ${ }^{7}$ German expert consensus guidelines from 2011 support many of these recommendations. ${ }^{8}$ Currently, ustekinumab and adalimumab are being evaluated for the treatment of pediatric psoriasis.

\section{Topicals}

A systematic review of calcipotriol in a total of 155 children concluded that it is an effective, well-tolerated option and gave it a Grade A recommendation. ${ }^{7}$

\section{Methotrexate}

In a retrospective series of 13 children with severe plaque psoriasis treated with low-dose once-weekly methotrexate, 11 achieved clearance and 2 stopped treatment due to elevated liver enzymes. Obesity was suggested as a relative contraindication due to the higher risk of fatty liver, which could increase methotrexate hepatotoxicity. ${ }^{9}$ A retrospective review of 24 children with severe psoriasis (17 with plaque psoriasis) obtained similar responses, with only mild adverse effects. ${ }^{10}$

\section{Phototherapy}

In the largest study to date, a 2011 retrospective review of 88 patients with psoriasis treated with NB-UVB, $51 \%$ of patients achieved clearance while $41 \%$ had good response (at least $75 \%$ improvement). ${ }^{11}$ The therapy was well tolerated, with mainly mild adverse effects in a median follow up of 3 years. ${ }^{11}$ Several smaller retrospective studies obtained similar results. ${ }^{12-14}$

\section{Addition to section on page 48}

\section{Pregnancy}

\section{Psoriasis Treatment Prior To or During Pregnancy}

The treatment options and risk classifications for the use of major psoriasis therapies in pregnant patients are summarized in Table 2 in the 2009 Canadian Guidelines for the Management of Plaque Psoriasis. In a recent review of treatment options in pregnant or lactating women, the Medical Board of the National Psoriasis Foundation largely supported the assessments in Table 2, with the notable exceptions of calcipotriol and anthralin, which the authors did not recommend as risks in pregnancy have not been reported. Their consensus first-line therapy for limited psoriasis in pregnancy is low- to moderate-potency topical corticosteroids, followed by second-line NB-UVB, and TNF inhibitors or cyclosporine "with caution" as a third-line option. ${ }^{15}$

\section{References}

1. Augustin M, Glaeske G, Radtke MA, et al. Epidemiology and comorbidity of psoriasis in children. $\mathrm{Br} J$ Dermatol. 2010;162(3):633-636.

2. Koebnick C, Black MH, Smith N, et al. The association of psoriasis and elevated blood lipids in overweight and obese children. J Pediatr. 2011;159(4):577-583.

3. Goldminz AM, Buzney CD, Kim N, et al. Prevalence of the metabolic syndrome in children with psoriatic disease. Pediatr Dermatol. 2013;30(6):700-705.

4. Jensen P, Zachariae C, Iversen L, et al. Cardiovascular risk factors in children and adolescents with psoriasis: a case-control study. Acta Derm Venereol. 2014;94(1):76-78.

5. Paller AS, Mercy K, Kwasny MJ, et al. Association of pediatric psoriasis severity with excess and central adiposity: an international cross-sectional study. JAMA Dermatol. 2013;149(2):166-176.

6. Bryld LE, Sorensen TI, Andersen KK, Jemec GB, Baker JL. High body mass index in adolescent girls precedes psoriasis hospitalization. Acta Derm Venereol. 2010;90(5):488-493. 
7. de Jager ME, de Jong EM, van de Kerkhof PC, et al. Efficacy and safety of treatments for childhood psoriasis: a systematic literature review. J Am Acad Dermatol. 2010;62(6):1013-1030.

8. Sticherling M, Augustin M, Boehncke WH, et al. Therapy of psoriasis in childhood and adolescence-a German expert consensus. J Dtsch Dermatol Ges. 2011;9(10):815-823.

9. Collin B, Vani A, Ogboli M, et al. Methotrexate treatment in 13 children with severe plaque psoriasis. Clin Exp Dermatol. 2009;34(3):295-298.

10. Kaur I, Dogra S, De D, et al. Systemic methotrexate treatment in childhood psoriasis: further experience in 24 children from India. Pediatr Dermatol. 2008;25(2):184-188.

11. Pavlovsky M, Baum S, Shpiro D, et al. Narrow band UVB: is it effective and safe for paediatric psoriasis and atopic dermatitis? J Eur Acad Dermatol Venereol. 2011;25(6): 727-729.

12. Ersoy-Evans S, Altaykan A, Sahin S, et al. Phototherapy in childhood. Pediatr Dermatol. 2008;25(6):599-605.

13. Kortuem KR, Davis MD, Witman PM, et al. Results of Goeckerman treatment for psoriasis in children: a 21-year retrospective review. Pediatr Dermatol. 2010;27(5):518-524.

14. Zamberk P, Velazquez D, Campos M, et al. Paediatric psoriasis-narrowband UVB treatment. J Eur Acad Dermatol Venereol. 2010;24(4):415-419.

15. Bae YS, Van Voorhees AS, Hsu S, et al. Review of treatment options for psoriasis in pregnant or lactating women: from the Medical Board of the National Psoriasis Foundation. $J$ Am Acad Dermatol. 2012;67(3):459-477. 


\section{Chapter 8: Exacerbation and Flare of Psoriasis}

Addition to subsection on page 58

\section{New-Onset Psoriasis}

\section{Infection}

Tonsillectomy has been suggested for patients with repeated streptococcal infections and guttate flares as a possible means to prevent recurrence of these episodes or progression to plaque psoriasis. ${ }^{6}$ However, any benefits of such an approach remain speculative. A recent randomized, blinded, prospective study found sustained improvement in $86 \%$ (13 of 15) of patients with chronic plaque psoriasis after tonsillectomy, with a $30 \%$ to $90 \%$ reduction in disease severity after 2 years of follow-up. Individual improvement correlated closely with decreases in circulating $\mathrm{T}$ cells that cross-react with streptococcal $\mathrm{M}$ protein and skin keratin. Levels of these cells in tonsils at removal correlated with blood levels, suggesting that tonsillectomy may be beneficial because tonsils play a role in psoriasis pathogenesis. ${ }^{1}$ These results remain to be confirmed by further studies.

\section{TNF Inhibitors}

When used to treat rheumatoid arthritis and other noncutaneous inflammatory disorders, the TNF inhibitors (infliximab, adalimumab, certolizumab, golimumab, and etanercept) have each been shown (although uncommonly) to induce psoriasis in individuals with neither a personal nor a family history of the disease. Recent systematic reviews of published cases found that the majority (70\%-85\%) were new in onset. ${ }^{2,3}$ Lesions appeared from 2 weeks to 9 years after starting therapy. ${ }^{3}$ Morphological findings were mainly pustular or plaque psoriasis, with a minority of guttate type. No specific demographic characteristics, primary diseases, or TNF inhibitors correlated with the eruptions. ${ }^{2,3}$

Most patients were successfully managed with topical corticosteroids or vitamin D analogues, without stopping TNF inhibitors. In some cases, switching to another TNF inhibitor partially or completely resolved the lesions, but this treatment change aggravated the lesions in other cases. Among patients who discontinued TNF inhibitors, mostbut not all-achieved complete resolution. ${ }^{2,3}$ The IL-12/23 inhibitor ustekinumab has also been used successfully to treat TNF inhibitor-induced psoriasis. ${ }^{4}$

\section{Addition to subsection on page 59}

\section{Rebound}

Rapid withdrawal of topical or systemic corticosteroids has been reported to trigger rebound psoriasis, including pustular or erythrodermal types. ${ }^{5}$ No major guidelines for psoriasis or other inflammatory diseases recommend systemic corticosteroids. ${ }^{6}$ Recent population-based studies found that these agents are among the most commonly prescribed systemic drugs. ${ }^{7,8}$ However, a review of literature dating back to 1950 found relatively few reports of rebound or exacerbation due to systemic corticosteroid withdrawal — far fewer than might be expected given widespread use - and noted a lack of controlled trials to assess safety or efficacy of these agents in psoriasis. ${ }^{9}$

\section{New section to add before Conclusion on page 60}

\section{Other Drug Triggers of Psoriasis}

Drug-induced psoriasis generally involves new-onset episodes in patients with no personal or family history of the disease and resolves once the inciting medication is halted. Lithium and interferon are strongly implicated in triggering psoriasis. ${ }^{5,10}$ Psoriasiform eruptions are the most frequently reported skin-related adverse event for $\beta$-blockers, occurring within 1 to 18 months of initiation. New-onset cases generally resolve once $\beta$-blockers are withdrawn, whereas exacerbations tend to improve without completely dissipating. ${ }^{5}$ Lithium has been associated with drug-induced psoriasis with a mean latency of 48 weeks, but exacerbation of preexisting disease may be more common, occurring on average 20 weeks after starting therapy. ${ }^{10}$

Other drugs that are reported to induce or exacerbate psoriasis include antimalarials (eg, chloroquine), tetracyclines, angiotensin-converting enzyme inhibitors, angiotensin II receptor blockers, nonsteroidal anti-inflammatory drugs (NSAIDs), terbinafine, and benzodiazepines. ${ }^{5,10}$ However, case-control studies of new-onset psoriasis in a large database in the United Kingdom found no association with $\beta$-blockers or other antihypertensives for any duration of use. ${ }^{11}$ Notably, only a slightly higher risk (adjusted odds ratio [OR] 1.68) of exacerbation was found with long-term lithium use ( $\geq 5$ prescriptions). ${ }^{12}$ 


\section{Recommendations (page 60)}

All new recommendations or modifications to existing recommendations are indicated in bold.

\begin{tabular}{lc} 
Recommendation and Level of Evidence & Grade of Recommendation \\
\hline In patients who develop new-onset plaque, pustular, or guttate psoriasis while receiving TNF inhibitors & Grade D \\
for nondermatological conditions, the psoriasis should, if possible, be controlled with topical agents & \\
(calcipotriol, corticosteroids, or both), with or without phototherapy, while maintaining anti-TNF & \\
therapy (Refs. 2, 3, LoE 3). & \\
If new-onset psoriasis does not respond to conventional psoriasis therapy, consider switching to & Grade D \\
another TNF inhibitor (Refs. 2, 3, LoE 3) or to an IL inhibitor (Ref. 4, LoE 3). & Grade D \\
In severe, erythrodermic, and/or intractable cases, TNF inhibitors should be halted (Refs. 2, \\
3, LoE 3).
\end{tabular}

\section{References}

1. Thorleifsdottir RH, Sigurdardottir SL, Sigurgeirsson B, et al. Improvement of psoriasis after tonsillectomy is associated with a decrease in the frequency of circulating $T$ cells that recognize streptococcal determinants and homologous skin determinants. J Immunol. 2012;188(10):5160-5165.

2. Collamer AN, Battafarano DF. Psoriatic skin lesions induced by tumor necrosis factor antagonist therapy: clinical features and possible immunopathogenesis. Semin Arthritis Rheum. 2010;40(3):233-240.

3. Denadai R, Teixeira FV, Steinwurz F, et al. Induction or exacerbation of psoriatic lesions during anti-TNF-alpha therapy for inflammatory bowel disease: a systematic literature review based on 222 cases. J Crohns Colitis. 2013;7(7):517-524.

4. Tillack C, Ehmann LM, Friedrich M, et al. Anti-TNF antibodyinduced psoriasiform skin lesions in patients with inflammatory bowel disease are characterised by interferon- $\gamma$-expressing Th1 cells and IL-17A/IL-22-expressing Th17 cells and respond to anti-IL-12/IL-23 antibody treatment. Gut. 2014;63(4):567-577.

5. Kim GK, DelRosso JQ. Drug-provoked psoriasis: is it druginduced or drug aggravated? Understanding pathophysiology and clinical relevance. J Clin Aesthet Dermatol. 2010;3(1):32-38.
6. Semble AL, Davis SA, Feldman SR. Lack of information in current guidelines regarding systemic corticosteroids in inflammatory diseases. Dermatol Online J. 2014;20(3).

7. Augustin M, Schafer I, Reich K, et al. Systemic treatment with corticosteroids in psoriasis - health care provision far beyond the S3-guidelines. J Dtsch Dermatol Ges. 2011;9(10):833-838.

8. Al-Dabagh A, Al-Dabagh R, Davis SA, et al. Systemic corticosteroids are frequently prescribed for psoriasis. J Cutan Med Surg. 2014;18:1-5.

9. Mrowietz U, Domm S. Systemic steroids in the treatment of psoriasis: what is fact, what is fiction? J Eur Acad Dermatol Venereol. 2013;27(8):1022-1025.

10. Rongioletti F, Fiorucci C, Parodi A. Psoriasis induced or aggravated by drugs. J Rheumatol Suppl. 2009;83:59-61.

11. Brauchli YB, Jick SS, Curtin F, et al. Association between beta-blockers, other antihypertensive drugs and psoriasis: population-based case-control study. $\mathrm{Br} J$ Dermatol. 2008;158(6):1299-1307.

12. Brauchli YB, Jick SS, Curtin F, et al. Lithium, antipsychotics, and risk of psoriasis. J Clin Psychopharmacol. 2009;29(2): 134-140. 


\section{Chapter 9: Management of Facial, Flexural, and Genital Psoriasis}

\section{Addition to section on page 63}

\section{Management}

The TCIs pimecrolimus and tacrolimus were effective and well tolerated in trials of up to 16 weeks in facial, flexural, and genital psoriasis, $,, 6,6,10-12,1-3$ although these agents are not approved for these indications or, indeed, for any form of psoriasis. Tacrolimus also demonstrated safety and efficacy in a 6-month pilot study in pediatric patients. ${ }^{4}$
Because vitamin $\mathrm{D}_{3}$ analogues can cause irritation and erythema, calcipotriol is not approved for use on the face or intertriginous areas, while calcitriol carries a warning against facial use. Nevertheless, both agents have been used successfully for facial and flexural psoriasis, with calcitriol showing greater efficacy and tolerability than calcipotriol..$^{-}$A small, double-blind, comparative trial between tacrolimus and calcitriol in facial and genital psoriasis found that both were well tolerated, but tacrolimus was more effective. ${ }^{2}$

\section{Recommendations (page 63)}

All new recommendations or modifications to existing recommendations are indicated in bold.

Recommendation and Level of Evidence

Grade of Recommendation

Topical calcineurin inhibitors ( $0.1 \%$ tacrolimus ointment or $1 \%$ pimecrolimus cream) may be used

Grade B

for facial, flexural, or genital areas (Ref. 5, LoE I+; Ref. 6, LoE I+; Refs. II, I2, LoE 2+; Ref. 2,

LoE I+; Refs. I, 3, LoE 2+).

\section{References}

1. Bissonnette R, Nigen S, Bolduc C. Efficacy and tolerability of topical tacrolimus ointment for the treatment of male genital psoriasis. J Cutan Med Surg. 2008;12(5):230-234.

2. Liao YH, Chiu HC, Tseng YS, et al. Comparison of cutaneous tolerance and efficacy of calcitriol 3 microg $\mathrm{g}(-1)$ ointment and tacrolimus $0.3 \mathrm{mg} \mathrm{g}(-1)$ ointment in chronic plaque psoriasis involving facial or genitofemoral areas: a double-blind, randomized controlled trial. Br J Dermatol. 2007;157(5):1005-1012.

3. Jacobi A, Braeutigam M, Mahler V, et al. Pimecrolimus $1 \%$ cream in the treatment of facial psoriasis: a 16-week open-label study. Dermatology. 2008;216(2):133-136.

4. Brune A, Miller DW, Lin P, et al. Tacrolimus ointment is effective for psoriasis on the face and intertriginous areas in pediatric patients. Pediatr Dermatol. 2007;24(1):76-80. 


\section{Chapter 10: Management of Nail Psoriasis}

Addition to section on page 65-67

\section{Presentation and Evaluation of Nail Psoriasis}

The NAPSI requires physicians to score each nail based on the nail matrix and nail bed parameters described in Table 1. However, a case-control study reported that leukonychia was seen more often in controls than in psoriatic patients. Thus, the authors suggested removing leukonychia from the NAPSI and replacing it with Beau lines, which were more common in nail psoriasis. ${ }^{1}$

\section{Management of Nail Psoriasis}

Certain treatments may increase the risk of onychomycosis. A prospective trial of patients with negative fungal scrapings randomized to infliximab, etanercept, or adalimumab found that those patients receiving infliximab had a significantly higher rate of onychomycosis than controls ( $33 \%$ vs $14 \%){ }^{2}$

\section{Topical Therapies}

Several newer topical options are promising. In an openlabel study in 6 patients, tazarotene $0.1 \%$ hydrophilic ointment under occlusion produced a statistically significant improvement (88\%) in the NAPSI score after 6 months, particularly in subungual hyperkeratosis, onycholysis, and associated pain. Unlike earlier trials of tazarotene under occlusion, no adverse effects were observed. ${ }^{3}$

Another potentially useful treatment is a water-soluble nail lacquer containing hydroxypropyl chitosan, horsetail (Equisetum arvense) extract, and methylsulfonylmethane. It was well tolerated and effective in reducing signs and symptoms of nail psoriasis (especially pitting, leukonychia and onycholysis) after 6 months in an open-label trial of 30 patients. ${ }^{4}$

Although topical tacrolimus is only indicated for moderate to severe atopic dermatitis, ${ }^{5}$ there are reports of its successful use in various forms of nail dystrophy. ${ }^{6}$ An open-label study in 21 patients of tacrolimus $0.1 \%$ ointment showed significant improvement in NAPSI scores of treated versus untreated nails after 12 weeks, with complete clinical resolution in 36 of 90 treated nails. One patient withdrew due to acute paronychia, but no other adverse effects were reported. ${ }^{6}$

A 2013 Cochrane review of interventions for nail psoriasis found no significant differences in RCTs comparing topical 5-fluorouracil versus vehicle, tazarotene versus clobetasol, and calcipotriol versus betamethasone. However, as the authors noted, "The evidence for the use of topical treatments is inconclusive and of poor quality; however, this does not imply that they do not work."

New section to add on page 67

\section{Pulse Dye Laser}

Pulse dye laser (PDL) is the accepted first-line therapy for cutaneous vascular disorders and is widely used for a variety of nonvascular conditions, including psoriasis. ${ }^{8}$ A 2013 systematic review of PDL for inflammatory skin diseases deemed the evidence in nail psoriasis to be insufficient to reach a conclusion, ${ }^{9}$ as it consisted of 2 uncontrolled studies, 1 of which showed a statistically significant decrease in NAPSI score with PDL. ${ }^{10,11}$ Since then, a study comparing different pulse durations ( $6 \mathrm{~ms}, 9 \mathrm{~J} / \mathrm{cm}^{2}$ vs $\left.0.45 \mathrm{~ms}, 6 \mathrm{~J} / \mathrm{cm}^{2}\right)$ in nail psoriasis found both types were equally effective and significantly reduced NAPSI scores after 6 months. ${ }^{12}$ Another study, an intrapatient controlled trial of PDL and tazarotene versus tazarotene control, showed significantly improved overall and nail matrix modified NAPSI scores after 6 months for the PDL arm versus control. ${ }^{13}$

Addition to section on page 67

\section{Systemic Therapies}

A randomized comparison of systemic methotrexate versus systemic cyclosporine in patients with nail involvement in moderate to severe plaque psoriasis found both agents moderately effective in reducing mean NAPSI scores after 6 months. Methotrexate reduced scores by $43.3 \%$ and cyclosporine by $37.2 \%$, with no significant differences by the end of treatment. However, methotrexate significantly reduced the nail matrix but not the nail bed score, while cyclosporine had the opposite result: The nail bed score was significantly lower but the nail matrix score was unchanged. ${ }^{14}$

Systemic low-dose acitretin $(0.2-0.3 \mathrm{mg} / \mathrm{kg} / \mathrm{d})$ demonstrated benefits in an open trial in isolated nail psoriasis, reducing mean NAPSI score by $41 \%$ and resulting in complete or almost complete clearing of lesions in $25 \%$, moderate improvement in $25 \%$, and mild improvement in $33 \%$ of patients. ${ }^{15}$

A retrospective analysis of this trial found that among patients who received infliximab for 1 full year, those with $75 \%$ or higher improvement in skin psoriasis had a mean 
decrease of $78 \%$ in nail psoriasis NAPSI scores, and $56 \%$ of these patients achieved complete nail clearance. ${ }^{16}$

In a pooled analysis of patients randomized to either continuous or paused etanercept therapy for 1 year, the mean NAPSI score improved by $51 \%$, and $30 \%$ of these patients reported no nail psoriasis at the end of the trial. ${ }^{17} \mathrm{~A}$ prospective, randomized trial of etanercept, either twice weekly for 12 weeks followed by once weekly for 12 weeks, or once weekly for 24 weeks, found that both regimens significantly improved mean NAPSI scores. By week 24, a majority of patients on either regimen had achieved $75 \%$ or greater improvement in NAPSI scores. ${ }^{18}$

A case series of patients given adalimumab for 24 weeks showed significant improvements in mean NAPSI scores with minimal side effects. ${ }^{19}$ In a post hoc analysis of a 16-week RCT of adalimumab in patients with nail involvement, a larger proportion attained $50 \%$ or greater NAPSI improvement in the adalimumab group (56.5\%) versus placebo $(12.5 \%){ }^{20}$

In an RCT of golimumab in PsA, patients with nail psoriasis in the treatment arm showed significant improvement in mean NAPSI and PGA of nail psoriasis versus placebo after 24 weeks. ${ }^{21}$
An RCT of ustekinumab measured NAPSI improvement as a secondary outcome after 12 and 64 weeks and found that score improvement was nonsignificant at 12 weeks for $45-\mathrm{mg}$ and $90-\mathrm{mg}$ doses and was considerable at 64 weeks $(>50 \%$ for both doses, no $P$ value reported) versus placebo. ${ }^{22}$ In 2014, an ustekinumab RCT designed to prospectively assess nail psoriasis showed significant improvement in NAPSI scores after 12 weeks for 45-mg and 90-mg doses versus placebo and showed continued improvement for both doses after 24 weeks. ${ }^{23}$

Few trials have compared the efficacy of TNF inhibitors versus older systemic therapies. A retrospective case series of patients receiving various systemics (acitretin, methotrexate, cyclosporin, PUVA, NB-UVB, retinoid + PUVA [RePUVA], retinoid + NB-UVB [ReNB-UVB], infliximab, efalizumab, etanercept, adalimumab) found that all except NB-UVB significantly improved mean NAPSI scores at 12 , 24 , and 48 weeks. Biologic therapies produced significantly greater percentage change in NAPSI scores than older systemics at all time points. ${ }^{24}$ The 2013 Cochrane review concluded that infliximab and golimumab but not ustekinumab demonstrated benefits over placebo controls. ${ }^{7}$

Addition to Table 2 on page 68

Table 2. Therapeutic Options for Managing Nail Psoriasis.

\begin{tabular}{ll}
\hline Type of Therapy & \multicolumn{1}{c}{ Important Contraindications and Therapeutic Considerations } \\
\hline PDL & $\begin{array}{l}\text { PDL was effective and well tolerated in several small case series. Adverse effects were mild and included transient } \\
\text { pain, purpura, and hyperpigmentation }{ }^{10-13}\end{array}$ \\
Biologic agents & $\begin{array}{l}\text { A number of RCTs of various biologics for moderate to severe plaque psoriasis or PsA demonstrated that patients } \\
\text { with concomitant nail involvement had significantly improved NAPSI scores after } 12 \text { to } 64 \text { weeks of therapy. Nail } \\
\text { benefits generally accrued more slowly than skin or joint improvement. }{ }^{16-18,20-23}\end{array}$ \\
\hline
\end{tabular}

\section{Recommendations (page 69)}

All new recommendations or modifications to existing recommendations are indicated in bold.

Pulse dye laser (PDL) treatment for 3 to 6 months may be clinically useful for nail psoriasis; more studies are needed (Ref. 9, LoE 2++; Refs. I0-I 2, LoE 3; Ref. 13, LoE 2-).

Patients with isolated nail psoriasis should not ordinarily be considered for systemic or phototherapy (LoE 4). However, in appropriate patients with other psoriatic manifestations, the presence of severe or intractable nail involvement may be a contributing factor in the decision to use any of the following to treat plaque psoriasis affecting other areas of the skin:

- Infliximab (Refs. 38, 39, LoE I++; Ref. 35, LoE 2-; Ref. I6, LoE I+)

- Etanercept (Refs. 17 and 18, LoE I+)

- Adalimumab (Ref. 19, LoE 3; Ref. 20, LoE I+)

- Golimumab (Ref. 2I, LoE I+)

- Ustekinumab (Refs. 22 and 23, LoE I+)

- Oral cyclosporine plus topical plus topical calcipotriol (Ref. 34, LoE 2+)

- Oral cyclosporine alone (Ref. 33, LoE I+; Ref. I4, LoE I-)

- Methotrexate (Ref. I4, LoE I-)

- Oral low-dose acitretin (0.2-0.3 $\mathrm{mg} / \mathbf{k g} / \mathrm{d})$ for isolated nail psoriasis (Ref. I 5, LoE 2-)

As a second-line option, topical tazarotene may be used (Refs. II, 14, LoE I-; Ref. 15, LoE 2-, Ref. 3, LoE 3). Topical tacrolimus may also be used (Ref. 6, LoE 2-).

\section{Grade C}

Grade D

Grade A

Grade B

Grade B

Grade B

Grade B

Grade C

Grade B

Grade C

Grade C

Grade C

Note: Previous recommendations for alefacept should be disregarded, as the product was withdrawn from the market in $20 \mathrm{I}$. 


\section{References}

1. van der Velden HM, Klaassen KM, van de Kerkhof P, et al. Fingernail psoriasis reconsidered: a case-control study. $\mathrm{J} \mathrm{Am}$ Acad Dermatol. 2013;69(2):245-252.

2. Al-Mutairi N, Nour T, Al-Rqobah D. Onychomycosis in patients of nail psoriasis on biologic therapy: a randomized, prospective open label study comparing etanercept, infliximab and adalimumab. Expert Opin Biol Ther. 2013;13(5): 625-629.

3. Fischer-Levancini C, Sanchez-Regana M, Llambi F, et al. Nail psoriasis: treatment with tazarotene $0.1 \%$ hydrophilic ointment. Actas Dermosifiliogr. 2012;103(8):725-728.

4. Cantoresi F, Sorgi P, Arcese A, et al. Improvement of psoriatic onychodystrophy by a water-soluble nail lacquer. $J$ Eur Acad Dermatol Venereol. 2009;23(7):832-834.

5. Astellas Pharma Canada, Inc. Protopic Tacrolimus Ointment. Canadian Product Monograph. Markham, ON: Astellas Pharma Canada; 2011.

6. De Simone C, Maiorino A, Tassone F, et al. Tacrolimus $0.1 \%$ ointment in nail psoriasis: a randomized controlled open-label study. J Eur Acad Dermatol Venereol. 2013;27(8):1003-1006.

7. de Vries AC, Bogaards NA, Hooft L, et al. Interventions for nail psoriasis. Cochrane Database Syst Rev. 2013;(1):CD007633.

8. Liu A, Moy RL, Ross EV, et al. Pulsed dye laser and pulsed dye laser-mediated photodynamic therapy in the treatment of dermatologic disorders. Dermatol Surg. 2012;38(3):351-366.

9. Erceg A, de Jong EM, van de Kerkhof PC, Seyger MM. The efficacy of pulsed dye laser treatment for inflammatory skin diseases: a systematic review. J Am Acad Dermatol. 2013;69(4):609-615.

10. Fernandez-Guarino M, Harto A, Sanchez-Ronco M, et al. Pulsed dye laser vs. photodynamic therapy in the treatment of refractory nail psoriasis: a comparative pilot study. J Eur Acad Dermatol Venereol. 2009;23(8):891-895.

11. Oram Y, Karincaoglu Y, Koyuncu E, et al. Pulsed dye laser in the treatment of nail psoriasis. Dermatol Surg. 2010;36(3):377-381.

12. Treewittayapoom C, Singvahanont $\mathrm{P}$, Chanprapaph K, et al. The effect of different pulse durations in the treatment of nail psoriasis with 595-nm pulsed dye laser: a randomized, doubleblind, intrapatient left-to-right study. $J$ Am Acad Dermatol. 2012;66(5):807-812.

13. Huang YC, Chou CL, Chiang YY. Efficacy of pulsed dye laser plus topical tazarotene versus topical tazarotene alone in psoriatic nail disease: a single-blind, intrapatient left-to-right controlled study. Lasers Surg Med. 2013;45(2):102-107.

14. Gumusel M, Ozdemir M, Mevlitoglu I, et al. Evaluation of the efficacy of methotrexate and cyclosporine therapies on psoriatic nails: a one-blind, randomized study. J Eur Acad Dermatol Venereol. 2011;25(9):1080-1084.

15. Tosti A, Ricotti C, Romanelli P, et al. Evaluation of the efficacy of acitretin therapy for nail psoriasis. Arch Dermatol. 2009;145(3):269-271.

16. Reich K, Ortonne JP, Kerkmann U, et al. Skin and nail responses after 1 year of infliximab therapy in patients with moderate-to-severe psoriasis: a retrospective analysis of the EXPRESS Trial. Dermatology. 2010;221(2):172-178.

17. Luger TA, Barker J, Lambert J, et al. Sustained improvement in joint pain and nail symptoms with etanercept therapy in patients with moderate-to-severe psoriasis. J Eur Acad Dermatol Venereol. 2009;23(8):896-904.

18. Ortonne JP, Paul C, Berardesca E, et al. A 24-week randomized clinical trial investigating the efficacy and safety of two doses of etanercept in nail psoriasis. Br J Dermatol. 2013;168(5): 1080-1087.

19. Rigopoulos D, Gregoriou S, Lazaridou E, et al. Treatment of nail psoriasis with adalimumab: an open label unblinded study. J Eur Acad Dermatol Venereol. 2010;24(5):530-534.

20. Poulin Y, Crowley JJ, Langley RG, et al. Efficacy of adalimumab across subgroups of patients with moderate-to-severe chronic plaque psoriasis of the hands and/or feet: post hoc analysis of REACH. J Eur Acad Dermatol Venereol. 2014;28(7):882-890.

21. Kavanaugh A, McInnes I, Mease P, et al. Golimumab, a new human tumor necrosis factor alpha antibody, administered every four weeks as a subcutaneous injection in psoriatic arthritis: twenty-four-week efficacy and safety results of a randomized, placebo-controlled study. Arthritis Rheum. 2009;60(4):976-986.

22. Igarashi A, Kato T, Kato M, et al. Efficacy and safety of ustekinumab in Japanese patients with moderate-to-severe plaque-type psoriasis: long-term results from a phase $2 / 3$ clinical trial. J Dermatol. 2012;39(3):242-252.

23. Rich P, Bourcier M, Sofen H, et al. Ustekinumab improves nail disease in patients with moderate-to-severe psoriasis: results from PHOENIX 1. Br J Dermatol. 2014;170(2):398-407.

24. Sanchez-Regana M, Sola-Ortigosa J, Alsina-Gibert, et al. Nail psoriasis: a retrospective study on the effectiveness of systemic treatments (classical and biological therapy). J Eur Acad Dermatol Venereol. 2011;25(5):579-586. 


\section{Chapter I I: Management of Scalp Psoriasis}

Addition to introduction on page 71

Topical corticosteroids, the mainstay of scalp psoriasis management, are therefore available as lotions, solutions, gels, sprays, shampoos, and foams. ${ }^{1}$

Clobetasol spray (not indicated for scalp in Canada) and shampoo are both effective and well tolerated with daily use for up to 4 weeks. ${ }^{2,3}$ Clobetasol shampoo maintenance therapy has also shown long-term efficacy and safety, with improved quality of life $(\mathrm{QoL}){ }^{3-5}$

Topical combination therapy with calcipotriol plus betamethasone dipropionate gel is well tolerated and more effective than either agent alone or vehicle after 8 weeks, with significant improvement as early as week $1 .^{6-9}$ Long-term safety and efficacy have been shown for up to 52 weeks. ${ }^{10}$ Combination therapy with calcipotriol plus betamethasone dipropionate gel is also safe and effective in the adolescent population. ${ }^{11}$

Monochromatic 308-nm excimer laser phototherapy has been used successfully in scalp psoriasis studies ${ }^{12-15}$ but is not widely available in Canada at present.

In a randomized, placebo-controlled study of moderate to severe psoriasis with scalp involvement, etanercept significantly improved scalp symptoms, with high patient satisfaction and safety. ${ }^{16}$ Other biologic therapies that have shown benefits in scalp symptoms in psoriasis patients include adalimumab $^{17}$ and ustekinumab. ${ }^{18,19}$

Addition to Table 1 on page 72

Table I. Topical and Phototherapeutic Options for Managing Scalp Psoriasis.

\begin{tabular}{lc}
\hline Type of Therapy & Important Contraindications and Therapeutic Considerations \\
\hline Corticosteroids & $\begin{array}{c}\text { Topical corticosteroids in lotions, solutions, shampoos, and foams designed for scalp application are } \\
\text { widely used in scalp psoriasis. A 6-month trial of maintenance therapy with clobetasol shampoo } \\
\text { confirmed its long-term safety and efficacy for this condition. }\end{array}$ \\
$\begin{array}{c}\text { Corticosteroid-vitamin } \mathrm{D}_{3} \\
\text { derivative combination therapy } \\
\text { Combination therapy can offer greater efficacy, safety, and tolerability than the individual } \\
\text { components. A fixed-combination calcipotriol plus betamethasone dipropionate gel is more } \\
\text { effective than either drug alone, with less irritation than calcipotriol monotherapy. } \\
\text { benefits have been documented in adolescents as well as adults. }{ }^{.1} \\
\text { UnB phose } \\
\text { Unlike traditional UVB phototherapy, targeted 308-nm excimer laser phototherapy can treat localized } \\
\text { lesions while sparing unaffected skin, allowing higher doses and potentially greater efficacy. }{ }^{14}\end{array}$ \\
\hline
\end{tabular}

\section{Recommendations (page 73)}

All new recommendations or modifications to existing recommendations are indicated in bold.

Moderately potent to very potent topical corticosteroids and calcipotriol are all appropriate topical treatments for mild to moderate scalp psoriasis.

Suitable agents include the following:

- Betamethasone dipropionate lotion, clobetasol propionate solution or spray, betamethasone valerate solution, or betamethasone valerate foam (Refs. 16, 17, LoE I++; Refs. 18, 19, LoE I+; Ref. I, LoE 2-)

- Clobetasol propionate shampoo (Ref. 20, LoE I++; Ref. 2I, LoE I+; Ref. 2, LoE I+; Ref. 3, LoE I++) Grade A

- Amcinonide lotion or fluocinonide (Ref. 22, LoE I++; Ref. 23, LoE I+)

- Calcipotriol solution (Refs. 24, 25, LoE I+)

- Combination therapy with calcipotriol/betamethasone dipropionate gel (Refs. 6- 8, I0, LoE I++)

For scalp psoriasis resistant to topical therapies, 308-nm excimer laser phototherapy may be considered where available (Refs. I2, 13, I5, LoE 2+).

In severe cases, systemic therapies may be considered.'

These include the following:

- Traditional agents (methotrexate, cyclosporine, or, for suitable patients, acitretin) (LoE 4)

Recommendation

- The biologic agents etanercept (Ref. I0, LoE I-; Ref. I6, LoE I+) adalimumab (Ref. I7, LoE I-), and ustekinumab (Refs. 18, 19, LoE 3)

Note: Previous recommendations for alefacept should be disregarded, as the product was withdrawn from the market in $20 \mathrm{II}$. 


\section{References}

1. Andreassi L, Giannetti A, Milani M. Efficacy of betamethasone valerate mousse in comparison with standard therapies on scalp psoriasis: an open, multicentre, randomized, controlled, crossover study on 241 patients. Br J Dermatol. 2003;148(1):134-138.

2. Sofen H, Hudson CP, Cook-Bolden FE, et al. Clobetasol propionate $0.05 \%$ spray for the management of moderate-to-severe plaque psoriasis of the scalp: results from a randomized controlled trial. J Drugs Dermatol. 2011;10(8):885-892.

3. Poulin Y, Papp K, Bissonnette R, et al. Clobetasol propionate shampoo $0.05 \%$ is efficacious and safe for long-term control of moderate scalp psoriasis. J Dermatolog Treat. 2010;21(3): 185-192.

4. Tan J, Thomas R, Wang B, et al. Short-contact clobetasol propionate shampoo $0.05 \%$ improves quality of life in patients with scalp psoriasis. Cutis. 2009;83(3):157-164.

5. Bovenschen HJ, Van de Kerkhof PC. Treatment of scalp psoriasis with clobetasol-17 propionate $0.05 \%$ shampoo: a study on daily clinical practice. J Eur Acad Dermatol Venereol. 2010;24(4):439-444.

6. Buckley C, Hoffmann V, Shapiro J, et al. Calcipotriol plus betamethasone dipropionate scalp formulation is effective and well tolerated in the treatment of scalp psoriasis: a phase II study. Dermatology. 2008;217(2):107-113.

7. Jemec GB, Ganslandt C, Ortonne JP, et al. A new scalp formulation of calcipotriene plus betamethasone compared with its active ingredients and the vehicle in the treatment of scalp psoriasis: a randomized, double-blind, controlled trial. $J$ Am Acad Dermatol. 2008;59(3):455-463.

8. Kragballe K, Hoffmann V, Ortonne JP, et al. Efficacy and safety of calcipotriol plus betamethasone dipropionate scalp formulation compared with calcipotriol scalp solution in the treatment of scalp psoriasis: a randomized controlled trial. $\mathrm{Br} J$ Dermatol. 2009;161(1):159-166.

9. Jemec GB, van de Kerkhof PC, Enevold A, et al. Significant one week efficacy of a calcipotriol plus betamethasone dipropionate scalp formulation. J Eur Acad Dermatol Venereol. 2011;25(1):27-32.

10. Luger TA, Cambazard F, Larsen FG, et al. A study of the safety and efficacy of calcipotriol and betamethasone dipropionate scalp formulation in the long-term management of scalp psoriasis. Dermatology. 2008;217(4):321-328.

11. Gooderham M, Debarre JM, Keddy-Grant J, et al. Safety and efficacy of calcipotriol plus betamethasone dipropionate gel in the treatment of scalp psoriasis in adolescents 12-17 years of age. Br J Dermatol. 2014;171(6):1470-1477.

12. Taylor CR, Racette AL. A 308-nm excimer laser for the treatment of scalp psoriasis. Lasers Surg Med. 2004;34(2):136-140.

13. Morison WL, Atkinson DF, Werthman L. Effective treatment of scalp psoriasis using the excimer $(308 \mathrm{~nm})$ laser. Photodermatol Photoimmunol Photomed. 2006;22(4): 181-183.

14. Wong JW, Kamangar F, Nguyen TV, et al. Excimer laser therapy for hairline psoriasis: a useful addition to the scalp psoriasis treatment algorithm. Skin Therapy Lett. 2012;17(5):6-9.

15. Al-Mutairi N, Al-Haddad A. Targeted phototherapy using 308 $\mathrm{nm}$ Xecl monochromatic excimer laser for psoriasis at difficult to treat sites. Lasers Med Sci. 2013;28(4):1119-1124.

16. Bagel J, Lynde C, Tyring S, et al. Moderate to severe plaque psoriasis with scalp involvement: a randomized, double-blind, placebo-controlled study of etanercept. J Am Acad Dermatol. 2012;67(1):86-92.

17. Thaçi D, Unnebrink K, Sundaram M, et al. Adalimumab for the treatment of moderate to severe psoriasis: subanalysis of effects on scalp and nails in the BELIEVE study. $J$ Eur Acad Dermatol Venereol. 2015;29(2):353-360.

18. Di Cesare A, Fargnoli MC, Peris K. Rapid response of scalp psoriasis to ustekinumab. Eur J Dermatol. 2011;21(6): 993-994.

19. Papadavid E, Ferra D, Koumaki D, et al. Ustekinumab induces fast response and maintenance of very severe refractory scalp psoriasis: results in two Greek patients from the psoriasis hospital-based clinic. Dermatology. 2014;228(2):107-111. 


\section{Chapter I2: Management of Palmoplantar Psoriasis}

\section{Addition to introduction on page 75}

A systematic review found that $56 \%$ of TNF inhibitorinduced or -exacerbated psoriasis cases were pustular, usually localized palmoplantar. ${ }^{1}$

New section to add on page 75

\section{Management of Palmoplantar Psoriasis and Palmoplantar Pustular Psoriasis}

With few controlled trials and only modest benefit from most treatments, many single and combination therapies may need to be attempted to achieve an adequate response. In a retrospective study of 114 patients with palmoplantar psoriasis or palmoplantar pustular psoriasis (PPP), about one-third showed marked improvement with topicals, while the rest were switched to traditional systemics and $40 \%$ needed multiple agents to achieve a response. ${ }^{2}$

Head-to-head trials are also limited. In a study comparing clobetasol propionate cream plus coal tar versus topical psoralen (8-methoxypsoralen 1\% lotion) and solar ultraviolet A (PUVA) therapy in palmoplantar psoriasis, both combinations produced similar mean improvements (50\%-75\%) and response rates (75\%-90\%). However, PUVA caused phototoxicity in one-fourth of patients, whereas no adverse effects were reported for the steroid and coal tar combination. ${ }^{3}$

A comparative trial of methotrexate in combination with various topicals and NB-UVB found that all were effective, but methotrexate/NB-UVB had the highest response rate and the lowest recurrence rate and was well tolerated. ${ }^{4}$

A 12-week comparative trial in palmoplantar psoriasis found that methotrexate had a faster treatment response and higher response rate than acitretin, although both were considered safe and effective overall. ${ }^{5}$

A recent consensus from the National Psoriasis Foundation made the following recommendations for PPP. For local therapy, first-line options were calcipotriol, corticosteroids, and topical PUVA; photodynamic therapy and tacrolimus were recommended for second-line use. For systemic therapy, first-line options included acitretin, cyclosporine, and RePUVA, while biologics were recommended for secondline therapy. ${ }^{6}$

\section{Addition to Table 1 on page 76}

Table I. Therapeutic Options for the Control of Palmoplantar Psoriasis.

\begin{tabular}{|c|c|}
\hline Type of Therapy & Important Contraindications and Therapeutic Considerations \\
\hline $\begin{array}{l}\text { Phototherapy and } \\
\text { photochemotherapy }\end{array}$ & $\begin{array}{l}\text { Topical PUVA is effective in palmoplantar psoriasis, but a Cochrane review of treatments for PPP concluded } \\
\text { that only oral PUVA has a proven (although modest) benefit in PPP., } \\
\text { In a prospective trial in patients with palmoplantar psoriasis, NB-UVB phototherapy yielded a } 60 \% \text { reduction in } \\
\text { severity, marked improvement in } 90 \% \text { of patients, and mild side effects and recurrence in one-third of patients. } \\
\text { Targeted UVB phototherapy with a } 308 \text {-nm excimer laser or lamp was beneficial in several open-label studies } \\
\text { in palmoplantar psoriasis and PPP, with improvements of } 50 \% \text { to } 100 \% .^{8-12} \text { Response rates were higher in } \\
\text { palmoplantar psoriasis than PPP. }{ }^{8} \text { Clinical improvement was rapid, usually seen by the fifth weekly session } \\
\text { and requiring } 10 \text { or more sessions for optimal response. }{ }^{9,10} \text { Common adverse effects included mild erythema, } \\
\text { itching, and transient hyperpigmentation. }\end{array}$ \\
\hline Vitamin $\mathrm{D}_{3}$ derivatives & $\begin{array}{l}\text { Calcipotriol has been used with moderate success in PPP, including acrodermatitis of Hallopeau, alone and in } \\
\text { combination with other topicals (tacrolimus, betamethasone). }{ }^{13-15}\end{array}$ \\
\hline Retinoids & $\begin{array}{l}\text { Oral retinoids have been used with a degree of success in the treatment of PPP, especially when combined with } \\
\text { PUVA therapy (RePUVA). }{ }^{.8-20} \text { RePUVA with acitretin (currently the only oral retinoid approved for use in } \\
\text { psoriasis in Canada) has not been studied systematically for palmoplantar psoriasis. } \\
\text { Acitretin produced the highest response rates in a retrospective cohort study of topicals and traditional } \\
\text { systemics: } 53 \% \text { of palmoplantar psoriasis and } 83 \% \text { of PPP patients. }{ }^{2} \text { Treatment response may be slow, with } \\
\text { only } 8 \% \text { of palmoplantar psoriasis patients responding by } 12 \text { weeks in a prospective study. }{ }^{5} \\
\text { Oral retinoids are contraindicated in women of childbearing potential unless suitable contraception is used. }\end{array}$ \\
\hline Cyclosporine & $\begin{array}{l}\text { In patients with PPP, treatment with cyclosporine brings about significant reductions in pustule formation, } \\
\text { erythema, infiltration, and scaling, as compared with placebo. }\end{array}$ \\
\hline
\end{tabular}




\section{Recommendations (page 77)}

All new recommendations or modifications to existing recommendations are indicated in bold.

First-line options for treating patients with plaque-type palmoplantar psoriasis include the following:

- Topical coal tar and salicylic acid under occlusion (Ref. I3, LoE 2+)

- Topical PUVA, including paint (Ref. 16, LoE 2++) and soak PUVA (Ref. 15, LoE 2++; Ref. 24, LoE 2+)

- Topical calcipotriol, with or without occlusion (Ref. I4, LoE 2-)

- Topical coal tar in combination with clobetasol propionate (Ref. 3, LoE 2-)

- NB-UVB (Ref. 4, LoE 2-)

- Targeted UVB phototherapy with an excimer laser or lamp (Refs. 7-9, I I, LoE 3)

Other options for which weaker evidence is available may also be considered, including moderate to ultrapotent corticosteroids (alone or in combination with calcipotriol), tazarotene, topical calcineurin inhibitors, and intralesional triamcinolone acetonide injection (LoE 4).

As second-line options, physicians may use systemic treatments, including the following:

- Acitretin (Ref. 2, LoE 2++; Ref. 5, LoE I-)

- Methotrexate (Ref. 4, LoE 2-)

- Cyclosporine (LoE 4)

- Infliximab (Ref. 19, LoE I-)

- Adalimumab (Ref. 20, LoE I-)

- Ustekinumab (Ref. 24, LoE 2-)

First-line topical options for PPP include the following:

- Triamcinolone acetonide or clobetasol propionate under occlusion (Ref. II, LoE 2+)

- Calcipotriol alone or combined with tacrolimus or betamethasone (Refs. I2-I4, LoE 3)

- Topical PUVA (Ref. 7, LoE 2++)

As second-line options in suitable patients with PPP, the physician may choose from the following:

- Cyclosporine (Refs. 16, 22, LoE I-)

- Acitretin (Ref. 2, LoE 2++; Ref. I2, LoE 3)

- Leflunomide (Ref. I7, LoE 3)

- Targeted UVB phototherapy with an excimer laser or lamp (Refs. 7-I I, LoE 3)

Third-line options for recalcitrant PPP include etanercept (Ref. 19, LoE I-) and ustekinumab (Refs. 2 I, 22, LoE 3; Ref. 23, LoE I-; Ref. 24, LoE 2-).

Intralesional triamcinolone acetonide injection, RePUVA with acitretin, and tonsillectomy may also be considered for suitable patients with PPP (Refs. 21, 25, 26, 27, LoE 3).

Note: Previous recommendations for alefacept should be disregarded, as the product was withdrawn from the market in $20 \mathrm{II}$.

\section{References}

1. Collamer AN, Battafarano DF. Psoriatic skin lesions induced by tumor necrosis factor antagonist therapy: clinical features and possible immunopathogenesis. Semin Arthritis Rheum. 2010;40(3):233-240.

2. Adisen E, Tekin O, Gulekon A, et al. A retrospective analysis of treatment responses of palmoplantar psoriasis in 114 patients. J Eur Acad Dermatol Venereol. 2009;23(7): 814-819.

3. Khandpur S, Sharma VK. Comparison of clobetasol propionate cream plus coal tar vs. topical psoralen and solar ultraviolet A therapy in palmoplantar psoriasis. Clin Exp Dermatol. 2011;36(6):613-616.

4. Gupta SK, Singh KK, Lalit M. Comparative therapeutic evaluation of different topicals and narrow band ultraviolet B therapy combined with systemic methotrexate in the treatment of palmoplantar psoriasis. Indian J Dermatol. 2011;56(2):165-170.

5. Janagond AB, Kanwar AJ, Handa S. Efficacy and safety of systemic methotrexate vs. acitretin in psoriasis patients with significant palmoplantar involvement: a prospective, randomized study. J Eur Acad Dermatol Venereol. 2013; 27(3):e384-e389.

6. Robinson A, Van Voorhees AS, Hsu S, et al. Treatment of pustular psoriasis: from the Medical Board of the National Psoriasis Foundation. J Am Acad Dermatol. 2012;67(2): 279-288.

7. Marsland AM, Chalmers RJ, Hollis S, et al. Interventions for chronic palmoplantar pustulosis. Cochrane Database Syst Rev. 2006;(1):CD001433.

8. Han L, Somani AK, Huang Q, et al. Evaluation of 308$\mathrm{nm}$ monochromatic excimer light in the treatment of psoriasis vulgaris and palmoplantar psoriasis. Photodermatology Photoimmunol Photomed. 2008;24(5):231-236.

9. Nistico SP, Saraceno R, Schipani C, et al. Different applications of monochromatic excimer light in skin diseases. Photomed Laser Surg. 2009;27(4):647-654.

10. Goldberg DJ, Chwalek J, Hussain M. 308-nm Excimer laser treatment of palmoplantar psoriasis. J Cosmet Laser Ther. 2011;13(2):47-49. 
11. Furuhashi T, Torii K, Kato H, et al. Efficacy of excimer light therapy $(308 \mathrm{~nm})$ for palmoplantar pustulosis with the induction of circulating regulatory T cells. Exp Dermatol. 2011;20(9):768-770.

12. Al-Mutairi N, Al-Haddad A. Targeted phototherapy using 308 $\mathrm{nm}$ Xecl monochromatic excimer laser for psoriasis at difficult to treat sites. Lasers Med Sci. 2013;28(4):1119-1124.

13. Piraccini BM, Tosti A, Iorizzo M, et al. Pustular psoriasis of the nails: treatment and long-term follow-up of 46 patients. $\mathrm{Br}$ J Dermatol. 2001;144(5):1000-1005.

14. Brill TJ, Elshorst-Schmidt T, Valesky EM, et al. Successful treatment of acrodermatitis continua of Hallopeau with sequential combination of calcipotriol and tacrolimus ointments. Dermatology. 2005;211(4):351-355.

15. Sotiriadis D, Patsatsi A, Sotiriou E, et al. Acrodermatitis continua of Hallopeau on toes successfully treated with a twocompound product containing calcipotriol and betamethasone dipropionate. J Dermatolog Treat. 2007;18(5):315-318.

16. Erkko P, Granlund H, Remitz A, et al. Double-blind placebocontrolled study of long-term low-dose cyclosporin in the treatment of palmoplantar pustulosis. Br J Dermatol. 1998; 139(6):997-1004.

17. Melwani PM, Penate Y, Guillermo N, et al. Leflunomide in the treatment of palmoplantar pustulosis. Arch Dermatol. 2009;145(11):1224-12246.

18. Bissonnette R, Poulin Y, Bolduc C, et al. Etanercept in the treatment of palmoplantar pustulosis. J Drugs Dermatol. 2008;7(10):940-946.

19. Bissonnette R, Poulin Y, Guenther L, et al. Treatment of palmoplantar psoriasis with infliximab: a randomized, double-blind placebo-controlled study. J Eur Acad Dermatol Venereol. 2011;25(12):1402-1408.

20. Leonardi C, Langley RG, Papp K, et al. Adalimumab for treatment of moderate to severe chronic plaque psoriasis of the hands and feet: efficacy and safety results from REACH, a randomized, placebo-controlled, double-blind trial. Arch Dermatol. 2011;147(4):429-436.

21. Gerdes S, Franke J, Domm S, et al. Ustekinumab in the treatment of palmoplantar pustulosis. $\mathrm{Br} J$ Dermatol. 2010;163(5):1116-1118.

22. Morales-Munera C, Vilarrasa E, Puig L. Efficacy of ustekinumab in refractory palmoplantar pustular psoriasis. $\mathrm{Br} \mathrm{J}$ Dermatol. 2013;168(4):820-824.

23. Bissonnette R, Nigen S, Langley R, et al. Increased expression of IL-17A and limited involvement of IL-23 in patients with palmo-plantar (PP) pustular psoriasis or PP pustulosis; results from a randomised controlled trial. J Eur Acad Dermatol Venereol. 2014;28(10):1298-1305.

24. Au SC, Goldminz AM, Kim N, et al. Investigator-initiated, open-label trial of ustekinumab for the treatment of moderate-to-severe palmoplantar psoriasis. J Dermatolog Treat. 2013;24(3):179-187.

25. Yokoyama M, Hashigucci K, Yamasaki Y. Effect of tonsillectomy in patients with pustulosis palmaris et plantaris. Acta Otolaryngol. 2004;124(9):1109-1110.

26. Takahara M. Clinical outcome of tonsillectomy for palmoplantar pustulosis and etiological relationship between palmoplantar pustulosis and tonsils. Adv Otorhinolaryngol. 2011;72:86-88. 


\section{Chapter I3: Social and Psychological Aspects of Psoriasis}

\section{Addition to Introduction on page 79}

Since the publication of the 2009 Canadian Guidelines for the Management of Plaque Psoriasis, more data on the disease burden of psoriasis in Canada specifically have come to light. A 2009 telephone survey of 500 Canadians living with the disease found that $35 \%$ considered psoriasis to be a substantial problem that affected their daily lives. ${ }^{1}$ A subsequent 2010 telephone survey of 514 Canadians with psoriasis found that psoriasis exerts at least a moderate effect on 39\% of individuals and a large effect on $15 \%$, according to Dermatology Life Quality Index (DLQI) results. ${ }^{2}$ A higher proportion of females than males surveyed reported feeling self-consciousness, while feelings of embarrassment and inconvenience appear the same for both genders. ${ }^{2}$

There is a link between disease improvement and quality of life (QoL) for patients - although the relationship is complex. A large study in patients with moderate to severe disease examined the relationship between achieving a PASI score of $75 \%$ versus PASI 90 or PASI $100 .{ }^{3}$ The study found that while all patients who achieved a PASI score greater than $75 \%$ also reported corresponding improvements in their health-related quality of life (HRQoL) outcome measures, those who achieved PASI 90 or 100 had greater improvements in the DLQI and Short Form $36 .{ }^{3}$ Patients achieving a PASI 90 to 100 were twice as likely to report no QoL impact from their disease, and those achieving PASI 100 were 3 times as likely to report no QoL impact from their disease compared with those achieving PASI 75 to $<90 .{ }^{3}$

Psychological comorbidity can worsen disease progression, and the treatment of depression would probably benefit clinical outcomes for patients. Likewise, delaying treatment for psoriasis could put patients at risk of worsening not only their clinical disease but also their associated HRQoL. ${ }^{4}$

\section{Addition to section on page 80}

\section{Effect of Psoriasis on Psychosocial Health}

Little is reported on the psychosocial effects of psoriasis in children. A 2012 review of this disease in pediatric patients reported that poor self-image and low self-esteem due to chronic illness may affect learning in early childhood and social involvement and acceptance in later years. ${ }^{5}$ Using the Children's Dermatology Life Quality Index (CDLQI), children reported psoriasis as having the most impact on QoL followed by atopic dermatitis. ${ }^{5}$
New section to add on page 80

\section{Economic Burden}

A review of studies on the costs of illness retrieved studies published from January 2002 to January $2010 .^{6}$ There is a dearth of literature on this issue, as only 7 studies were found. The data from the 7 studies indicate that direct costs were indeed higher than indirect costs. ${ }^{6}$ Hospitalization represented the most significant direct cost. The review confirms that indirect and direct costs increase with disease severity. The review also surmised that the intangible costs of psoriasis may be substantial, considering the degree of stigmatization perceived by patients and its impact on their QoL. On the costs of biologic agents, the review concludes that methods of cost-of-illness studies need to be harmonized and long-term economic burden and remission rate studies need to be considered before any judgments are made. ${ }^{6}$

An assessment of the economic burden of 90 Canadian patients diagnosed from 3 clinics located in British Columbia, Ontario, and Quebec represents the first of its kind to determine the economic burden of moderate to severe psoriasis in Canada. ${ }^{7}$ The study estimated that the mean annual cost of psoriasis was $\$ 7999$ per subject in $2008 .^{2}$ Direct costs were estimated to account for $57 \%$ of total annual costs, while $43 \%$ was due to a loss in productivity. ${ }^{7}$ The estimated annual cost to Canadian society was approximately $\$ 1.7$ billion. $^{7}$

New section to add on page 81

\section{Impact of Psoriasis Treatment on Quality of Life}

\section{Systemic Agents}

Since the publication of the 2009 Canadian Guidelines for the Management of Plaque Psoriasis, a slew of studies have examined the impact of specific treatments on QoL. Of note, more data on the impact of biologic therapies in particular have been published. A 2008 meta-analysis on the impact of biologic therapy on HRQoL found that all treatments in this class improved HRQoL compared with placebo. ${ }^{8}$

A 2012 review of the use of the DLQI as an outcome measure for QoL in psoriasis patients treated with biologic agents suggested that these agents vary in their impact on DLQI. ${ }^{9}$ In patients with moderate to severe disease, treatment with ustekinumab appeared to improve DLQI scores the most when compared with infliximab, etanercept, and adalimumab. ${ }^{9}$ The review, however, was limited by the inconsistency among the 
different methods used by the various studies reviewed (ie, duration of treatment, dosage, etc).

A study evaluating the results from two phase III clinical trials of ustekinumab concluded that this agent decreased sexual difficulties and improved HRQoL significantly. ${ }^{10}$ The study showed overall that sexual difficulties decreased as psoriasis improved. A subsequent data review of one of the phase III clinical trials corroborated that ustekinumab improved HRQoL in patients. ${ }^{11}$ Likewise, results from a phase II trial of ustekinumab indicated that therapy with this agent increased productivity, reduced work days missed, and improved work limitations when compared with placebo. ${ }^{10}$ However, further analysis would be required to clarify costs savings and economic benefits gained by using this biologic agent.

Most importantly, the data from these studies underscore the need to use both physician-reported and patient-reported measurement instruments to determine the full impact of treatment. $^{11}$

\section{Topical Agents}

Besides QoL studies for biologic agents, some more recent studies have been conducted on the impact of topical therapies on the QoL of psoriasis patients since the 2009 Guidelines. Scalp psoriasis is particularly difficult for patients and carries a significant psychosocial impact. A study on the use of calcipotriol/betamethasone in patients with scalp psoriasis found that the use of this combination significantly improved QoL compared with calcipotriol solution alone. ${ }^{12}$ A subsequent study confirmed these results using a QoL index derived from the DLQI but adapted specifically to the scalp (Scalp Life Quality Index) to measure outcomes. $^{13}$ The study found that this treatment could reduce the subjective burden of disease by $50 \%$ for all dimensions measured pertaining to QoL. ${ }^{13}$

In a review of 5 clinical trials that included more than 2000 patients, the collective data suggested that treatment with clobetasol propionate $0.05 \%$ spray was associated with significant improvements in QoL in patients with moderate to severe psoriasis. ${ }^{14}$

A phase IIb study of apremilast reported an improvement in HRQoL in patients with moderate to severe psoriasis. $^{15}$

\section{References}

1. Lynde CW, Poulin Y, Guenther L, et al. The burden of psoriasis in Canada: insights from the pSoriasis Knowledge IN Canada (SKIN) survey. J Cutan Med Surg. 2009;13(5): 235-252.
2. Papp K, Valenzuela F, Poulin Y, et al. Epidemiology of moderate-to-severe plaque psoriasis in a Canadian surveyed population. J Cutan Med Surg. 2010;14(4):167-174.

3. Revicki DA, William MK, Menter A, et al. Relationship between clinical response to therapy and health-related quality of life outcomes in patients with moderate to severe plaque psoriasis. Dermatology. 2008;216(3):260-270.

4. Papp KA, Signorovitch J, Ramakrishnan K, et al. Effects of adalimumab versus placebo on risk of symptom worsening in psoriasis and subsequent impacts on health-related quality-oflife: analysis of pooled data from two randomized, doubleblind, placebo-controlled, multicentre clinical trials. Clin Drug Investig. 2011;31(1):51-60.

5. Lin VW. Tough-skinned kids: identifying psychosocial effects of psoriasis and helping pediatric patients and families cope. J Pediatr Nurs. 2012;27(5):563-572.

6. Raho G, Koleva DM, Garattini L, et al. The burden of moderate to severe psoriasis: an overview. Pharmacoeconomics. 2012;30(11):1005-1013.

7. Levy AR, Davie AM, Brazier NC, et al. Economic burden of moderate to severe plaque psoriasis in Canada. Int J Dermatol. 2012;51(12):1432-1440.

8. Reich K, Sinclair R, Roberts G, et al. Comparative effects of biological therapies on the severity of skin symptoms and healthrelated quality of life in patients with plaque-type psoriasis: a meta-analysis. Curr Med Res Opin. 2008;24(5):1237-1254.

9. Basra MK and Hussain S. Application of the dermatology life quality index in clinical trials of biologics for psoriasis. Chin J Integr Med. 2012;18(3):179-185.

10. Reich K, Schenkel B, Zhao N, et al. Ustekinumab decreases work limitations, improves work productivity, and reduces work days missed in patients with moderate-to-severe psoriasis: results from PHOENIX 2. J Dermatolog Treat. 2011;22(6):337-347.

11. Lebwohl M, Papp K, Han C, et al. Ustekinumab improves health-related quality of life in patients with moderate-tosevere psoriasis: results from the PHOENIX 1 trial. $\mathrm{Br} J$ Dermatol. 2010;162(1):137-146.

12. Ortonne JP, Ganslandt C, Tan J, et al. Quality of life in patients with scalp psoriasis treated with calcipotriol/betamethasone dipropionate scalp formulation: a randomized controlled trial. J Eur Acad Dermatol Venereol. 2009;23(8):919-926.

13. Mrowietz U, Macheleidt O, Eicke C, et al. Effective treatment and improvement of quality of life in patients with scalp psoriasis by topical use of calcipotriol/betamethasone (Xamiol(R)gel): results. J Dtsch Dermatol Ges. 2011;9(10):825-831.

14. Menter MA, Caverney SW, Gottschalk RW, et al. Impact of clobetasol propionate $0.05 \%$ spray on health-related quality of life in patients with plaque psoriasis. J Drugs Dermatol. 2012;11(11):1348-1354.

15. Strand V, Florentino D, Hu C, et al. Improvements in patientreported outcomes with apremilast, an oral phosphodiesterase 4 inhibitor, in the treatment of moderate to severe psoriasis: results from a phase IIb randomized, controlled study. Health Qual Life Outcomes. 2013;11:82. 


\section{Chapter 14: Comorbidities}

Figure 1 depicts the different comorbidities discussed in this chapter of the addendum.

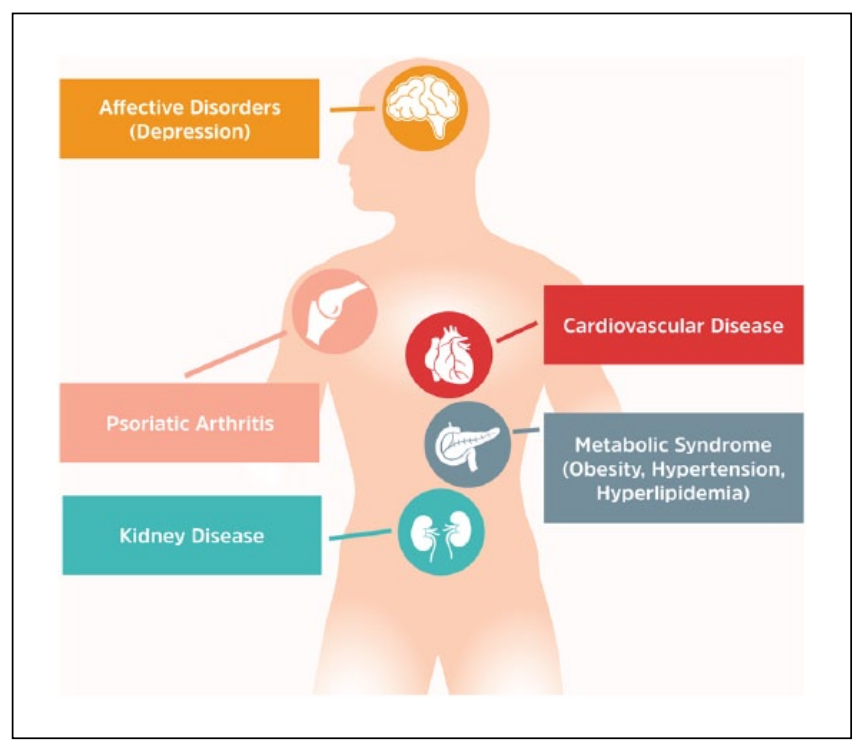

Figure I. Comorbidities in psoriasis.

Addition to section on page 84

\section{Affective Disorders}

The prevalence of depression in psoriasis patients varies considerably across studies, but a recent meta-analysis concluded that more than one-fourth had depressive symptoms and more than $10 \%$ were clinically depressed. Compared with controls, psoriasis patients had significantly more depression symptoms (SMD 1.16), had an OR of 1.57 (95\% CI, 1.40-1.76) for clinical depression (using International Classification of Diseases codes), and had an OR of 4.24 (95\% CI, 1.53-11.76) for antidepressant use. ${ }^{1}$

Growing evidence shows that TNF- $\alpha$ and other proinflammatory cytokines play a role in depression, suggesting that TNF inhibitors and other biologics could improve mood directly, not just by improving psoriasis. ${ }^{2}$ RCTs of etanercept, ${ }^{3}$ adalimumab, ${ }^{4}$ and ustekinumab ${ }^{5}$ have demonstrated benefits in reducing depressive symptoms associated with moderate to severe psoriasis, although it is not known whether the effects are direct or indirect.

Addition to section on page 85

\section{Cardiovascular Disease}

The association between psoriasis and type 2 diabetes was supported by a mixed retrospective-prospective cohort study showing a higher risk (RR 1.26; 95\% CI, 1.08-1.46) of diabetes in individuals under age $60{ }^{6}$

A large cross-sectional study from Israel showed a similar association between psoriasis and each of several components of the metabolic syndrome, including hypertension, hyperlipidemia, and obesity, as well as ischemic heart disease. ${ }^{19}$ A follow-up case-control study using the same Israeli database confirmed a significantly higher prevalence of hypertension in psoriasis patients. ${ }^{7}$

In a pooled retrospective analysis, the 10-year Framingham risk scores for psoriasis patients were $28 \%$ higher for coronary heart disease and $12 \%$ higher for stroke than in the general population. The risk scores were similar regardless of psoriasis severity. ${ }^{8}$

The association between psoriasis and obesity may be bidirectional: Obesity is a risk factor for onset of psoriasis and development of more severe forms, while psoriasis can lead to weight gain. ${ }^{9}$ Two RCTs on the effect of weight loss on psoriasis severity achieved mixed results. A 16 -week trial of a low-energy diet in 60 patients produced significantly more weight loss in the intervention group but only a trend for reduced psoriasis severity, ${ }^{9}$ while a 20 -week diet and exercise intervention trial in 303 patients achieved both significant weight loss and improved psoriasis severity. ${ }^{10}$

However, as previously discussed, many traditional cardiovascular disease (CVD) risk factors are more common in psoriasis patients. A meta-analysis of 14 cohort studies found inadequate controls for such confounders, making it difficult to quantify the risks due to psoriasis alone. Only severe psoriasis was associated with a higher CVD risk, due to greater incidence of myocardial infarction (MI), stroke, and allcause CVD mortality, and the relative risk was highest in the younger subgroup. ${ }^{11}$

A large US cohort study did not find a reduced risk of MI in patients with psoriasis receiving systemic therapies versus phototherapy, ${ }^{12}$ while a Danish cohort study found that biologic therapies and methotrexate decreased the risk of cardiovascular death, MI, and stroke. ${ }^{13}$ A population-based study from Taiwan showed that methotrexate protected against cerebrovascular disease, whereas systemic retinoids conferred no such benefit. ${ }^{14}$ The cohort data suggesting benefit are supported by prospective studies showing improvements in cardiovascular risk biomarkers with effective systemic therapy. ${ }^{15,16}$ 
This cardioprotective effect has not been reported with the anti-IL-12 and anti-IL-23 inhibitors. Long-term safety data for ustekinumab, with up to 5 years of follow-up, did not show any increased risk of cardiovascular events. ${ }^{17}$

\section{Addition to section on page 86}

\section{Psoriatic Arthritis}

Psoriatic arthritis (PsA) is an erosive arthritis occurring in up to $30 \%$ of psoriasis patients. ${ }^{35}$ This prevalence was confirmed in a large multinational study of rheumatologist-diagnosed PsA in psoriasis patients. Surprisingly, $41 \%$ of the patients with PsA had not been formally diagnosed previously. ${ }^{18}$ In a Canadian 4-year prospective study, the annual incidence rate of PsA was 1.87 cases per 100 psoriasis patients - considerably higher than earlier estimates from cumulative incidence. ${ }^{19}$

Ustekinumab is safe and effective in reducing signs and symptoms and preventing radiographic progression of active PsA, including in patients previously treated with TNF inhibitors or methotrexate. ${ }^{20-22}$

New section

\section{Kidney Disease}

Studies examining the risk of kidney disease in psoriasis patients have yielded conflicting results and have largely been small with a cross-sectional design. A large population-based cohort study that used electronic medical records from a database maintained by general practitioners in the United Kingdom included data on all patients with psoriasis aged 18 to 90 , collected prospectively from 2003 to September $2010 .^{23}$ In all, 136,529 patients with mild psoriasis and 7354 patients with severe psoriasis were matched to 689,702 patients unaffected by psoriasis. The adjusted hazard ratios (HRs) for chronic kidney disease (CKD) were 1.05 overall, 0.99 for mild psoriasis, and 1.93 for severe psoriasis patients. Age was a significant factor in the severe psoriasis patients, with an agespecific adjusted HR of 3.82 for patients aged 30 and 2.00 for patients aged 60. One limitation of the study (that was not noted by the authors) is that the use of angiotensin-converting enzyme inhibitors among patients - a key confounder - was not controlled for. The study concluded that independent of traditional risk factors for renal dysfunction, moderate to severe psoriasis is associated with an increased risk of CKD.

See Figure 1 for a depiction of the comorbidities discussed in this chapter of the addendum.

\section{Recommendations (page 87)}

All new recommendations or modifications to existing recommendations are indicated in bold.

\section{References}

1. Dowlatshahi EA, Wakkee M, Arends LR, et al. The prevalence and odds of depressive symptoms and clinical depression in psoriasis patients: a systematic review and meta-analysis. J Invest Dermatol. 2014;134(6):1542-1551.

2. Kannan S, Heller MM, Lee ES, et al. The role of tumor necrosis factor-alpha and other cytokines in depression: what dermatologists should know. J Dermatolog Treat. 2013;24(2):148-152.

3. Krishnan R, Cella D, Leonardi C, et al. Effects of etanercept therapy on fatigue and symptoms of depression in subjects treated for moderate to severe plaque psoriasis for up to 96 weeks. Br J Dermatol. 2007;157(6):1275-1277.

4. Menter A, Augustin M, Signorovitch J, et al. The effect of adalimumab on reducing depression symptoms in patients with moderate to severe psoriasis: a randomized clinical trial. $J \mathrm{Am}$ Acad Dermatol. 2010;62(5):812-818.

5. Langley RG, Feldman SR, Han C, et al. Ustekinumab significantly improves symptoms of anxiety, depression, and skin-related quality of life in patients with moderate-to-severe psoriasis: results from a randomized, double-blind, placebo-controlled phase III trial. J Am Acad Dermatol. 2010;63(3):457-465.

6. Li W, Han J, Hu FB, et al. Psoriasis and risk of type 2 diabetes among women and men in the United States: a populationbased cohort study. J Invest Dermatol. 2012;132(2):291-298.

7. Cohen AD, Weitzman D, Dreiher J. Psoriasis and hypertension: a case-control study. Acta Derm Venereol. 2010;90(1):23-26.

8. Kimball AB, Guerin A, Latremouille-Viau D, et al. Coronary heart disease and stroke risk in patients with psoriasis: retrospective analysis. Am J Med. 2010;123(4):350-357.

9. Jensen P, Zachariae C, Christensen R, et al. Effect of weight loss on the severity of psoriasis: a randomized clinical study. JAMA Dermatol. 2013;149(7):795-801.

10. Naldi L, Conti A, Cazzaniga S, et al. Diet and physical exercise in psoriasis: a randomized controlled trial. Br J Dermatol. 2014;170(3):634-642.

11. Samarasekera EJ, Neilson JM, Warren RB, et al. Incidence of cardiovascular disease in individuals with psoriasis: a systematic review and meta-analysis. J Invest Dermatol. 2013;133(10):2340-2346. 
12. Abuabara K, Lee H, Kimball AB. The effect of systemic psoriasis therapies on the incidence of myocardial infarction: a cohort study. Br J Dermatol. 2011;165(5):1066-1073.

13. Ahlehoff O, Skov L, Gislason G, et al. Cardiovascular disease event rates in patients with severe psoriasis treated with systemic anti-inflammatory drugs: a Danish real-world cohort study. J Intern Med. 2013;273(2):197-204.

14. Lan CC, Ko YC, Yu HS, et al. Methotrexate reduces the occurrence of cerebrovascular events among Taiwanese psoriatic patients: a nationwide population-based study. Acta Derm Venereol. 2012;92(4):349-352.

15. Boehncke S, Salgo R, Garbaraviciene J, et al. Effective continuous systemic therapy of severe plaque-type psoriasis is accompanied by amelioration of biomarkers of cardiovascular risk: results of a prospective longitudinal observational study. J Eur Acad Dermatol Venereol. 2011;25:1187-1193.

16. Montaudie H, Albert-Sabonnadiere C, Acquacalda E, et al. Impact of systemic treatment of psoriasis on inflammatory parameters and markers of comorbidities and cardiovascular risk: results of a prospective longitudinal observational study. J Eur Acad Dermatol Venereol. 2014;28(9):1186-1191.

17. Papp KA, Griffiths CE, Gordon K, et al. Long-term safety of ustekinumab in patients with moderate-to-severe psoriasis: final results from 5 years of follow-up. Br J Dermatol. 2013;168(4):844-854.

18. Mease PJ, Gladman DD, Papp KA, et al. Prevalence of rheumatologist-diagnosed psoriatic arthritis in patients with psoriasis in European/North American dermatology clinics. J Am Acad Dermatol. 2013;69(5):729-735.

19. Eder L, Chandran V, Shen H, et al. Incidence of arthritis in a prospective cohort of psoriasis patients. Arthritis Care Res 2011;63(4):619-622.

20. McInnes IB, Kavanaugh A, Gottlieb AB, et al. Efficacy and safety of ustekinumab in patients with active psoriatic arthritis: 1 year results of the phase 3 , multicentre, double-blind, placebo-controlled PSUMMIT 1 trial. Lancet. 2013;382(9894):780-789.

21. Ritchlin C, Rahman P, Kavanaugh A, et al. Efficacy and safety of the anti-IL-12/23 p40 monoclonal antibody, ustekinumab, in patients with active psoriatic arthritis despite conventional non-biological and biological anti-tumour necrosis factor therapy: 6-month and 1-year results of the phase 3, multicentre, double-blind, placebo-controlled, randomised PSUMMIT 2 trial. Ann Rheum Dis. 2014;73(6):990-999.

22. Kavanaugh A, Ritchlin C, Rahman P, et al. Ustekinumab, an anti-IL-12/23 p40 monoclonal antibody, inhibits radiographic progression in patients with active psoriatic arthritis: results of an integrated analysis of radiographic data from the phase 3, multicentre, randomised, double-blind, placebo-controlled PSUMMIT-1 and PSUMMIT-2 trials. Ann Rheum Dis. 2014;73(6):1000-1006.

23. Wan J, Wang S, Haynes K, et al. Risk of moderate to advanced kidney disease in patients with psoriasis: population based cohort study. BMJ. 2013;347:f5961. 


\section{Chapter 15: The Future Of Psoriasis Care}

\section{Addition to section on page 89}

\section{Standards of Care in Psoriasis Treatment}

With the increased recognition of quality-of-life issues for psoriasis patients has come a greater impetus for achieving more adequate control if this can be accomplished safely. For example, the 2012 German guidelines for the treatment of psoriasis outline the use of the PASI and DLQI in combination to incorporate QoL measures into clinical decision-making. ${ }^{1} \mathrm{~A}$ minimum target is one way to measure the successful achievement of treatment goals. In psoriasis, PASI 50 is usually the minimum goal. For DLQI, a change of 5 points is deemed clinically significant, while a DLQI of $0 / 1$ represents no meaningful impact on QoL. Most clinical studies use PASI 75 (achieved over 10-16 weeks) as a benchmark of successful therapy. Patients who achieve this goal generally have a corresponding improvement in their DLQI score as well. This is the rationale behind the twinning of PASI and DLQI to guide clinical decision making.

A modified Investigator's Global Assessment (IGA) tool has been used in clinical trials. ${ }^{2}$ The modified IGA is a static 5-point instrument to register a clinician's impression of disease severity from a score of 0 (clear) to 4 (severe). An evaluation of the tool sought to compare associations between IGA $0 / 1$ scores in responders to PASI scores. ${ }^{3}$ The study found that 5-point IGA was a valid and clinically useful measure of treatment benefit.

Another instrument used to measure treatment success is the Psoriasis Symptom Inventory (PSI) - a novel patientreported outcome measure that has also been used in clinical trial investigations. ${ }^{4}$ The PSI is an 8 -item measure that considers psoriasis symptoms such as itch, redness, scaling, burning, cracking, flaking, stinging, and pain. An analysis of the instrument as a measure of symptom severity in patients with moderate to severe disease found that it is a valid measure of symptom severity. ${ }^{5}$

Addition to section on page 90

\section{Canadian Implementation of Practices Used Elsewhere}

New formulations of existing products may have a more immediate impact on psoriasis care. For instance, foam preparations of corticosteroids appear to be better accepted by patients, relative to other formulations that are similar or identical in their active components. ${ }^{10,11}$ Foam preparations are now available in Canada.

\section{Priorities for Future Research}

\section{Prospects for More Head-to-Head Studies}

The first registered trial to compare two biologics (etanercept vs. the novel agent ustekinumab) began enrolling patients in 2007. In 2010, a new standard was established with the publication of the Active Comparator (CNTO1275/ Enbrel) Psoriasis Trial (ACCEPT). ${ }^{6}$ This study design included an active comparator arm, which enabled the direct comparison of 2 biologics. Since 2010, most new compounds have had at least 1 trial with an active comparator arm. This holds true for both novel biologics and emerging small molecules. Physicians can now make more informed decisions using the data from these head-to-head trials.

Revision of subsections on pages 90-91

\section{New Agents}

Antiangiogenesis as a therapeutic target. Angiogenesis has been suspected to contribute to the pathogenesis of psoriasis. Strong up-regulation of the angiogenic factor vascular endothelial growth factor (VEGF) has been shown to occur in psoriatic skin lesions. Investigation into the role of angiogenic factors in psoriasis is limited, but they are believed to accelerate angiogenesis in these patients. Thus, antiangiogenic peptides could prove to be useful and novel therapeutic tools in the treatment of psoriasis. A study in a mouse model using the low-molecular, pigment epithelium-derived factor (PEDF) peptide that is produced in human skin has been shown to exhibit antiangiogenic activity. ${ }^{7}$ In the study, local application of PEDF resulted in reduced acanthosis in psoriatic lesions and hyperplasia in normal skin. In addition, topical application of the PEDF peptide lessened the proliferation and inflammation found in psoriatic lesions. Similarly, a 2012 literature search for studies on VEGF antagonists found that VEGF inhibition is effective in the treatment of psoriasis in mice. ${ }^{8}$ Anti-VEGF therapies, such as bevacizumab, sunitinib, and sorafenib, are already being used in the treatment of malignant diseases and have been reported to induce disease remission in psoriasis. In addition, a few clinical case studies have suggested that VEGF antagonists may be useful 
in the management of psoriasis. Further clinical research will help determine the utility of these drugs in psoriasis.

Emerging small-molecule drugs. Efforts to develop novel, nonsteroidal topical therapies for mild psoriasis has led to the study of small-molecule drugs. One candidate, WBI-1001, was studied in patients with mild to moderate disease. ${ }^{9} \mathrm{WBI}-$ 1001 is classified as a nonsteroidal, anti-inflammatory, new chemical entity that works by inhibiting multiple proinflammatory cytokines and T-cell viability as well as infiltration processes. In a randomized, double-blind, placebo-controlled trial, patients in the treatment group received 1\% WB-1001 cream twice daily. At the end of 12 weeks, topical WB-1001 was found to induce rapid and significant improvement in Physician's Global Assessment (PGA) scores. Side effects were mild or moderate.

Protein kinase $\mathrm{C}$ (PKC) isoforms play important roles in the activation of $\mathrm{T}$ cells and other immune functions. The inhibition of $\mathrm{PKC}$ isoforms by AEB071 prevents the production of several cytokines. In a study on patients with moderate to severe psoriasis, the small-molecule and PKC inhibitor AEB071 induced a reduction of up to $69 \%$ in PASI scores after 2 weeks of treatment. ${ }^{10}$ Patients in this study were given oral doses of AEB071 of 50, 200, 400, and $600 \mathrm{mg} / \mathrm{d}$ for 2 weeks. This small-molecule drug has been noted to inhibit activation of $\mathrm{T}$ cells in a dose-dependent manner. No serious adverse events were reported.

Other small-molecule drugs work by inhibiting the pathway known as Janus kinase signal transducer and activator of transcription (JAK-STAT), which is a signalling pathway that is involved in the regulation of the immune system. These drugs include tofacitinib, ruxolitinib, and baricitinib.

A phase IIa, randomized, double-blind, vehicle-controlled trial investigated the utility of the Janus kinase (JAK) 3 inhibitor tofacitinib as a topical formulation in mild to moderate psoriasis. ${ }^{11}$ The primary endpoint of the study was percentage change in the Target Plaque Severity (TPS) score after 4 weeks. Patients using $2 \%$ tofacitinib ointment twice daily for 4 weeks showed statistically significant improvement compared with those using the vehicle alone. The topical therapy was also well tolerated.

A phase IIb study to assess the safety and efficacy of tofacitinib in the treatment of psoriasis found that short-term treatment (12 weeks) with an oral formulation of this JAK3 inhibitor resulted in significant improvement in moderate to severe cases of psoriasis. ${ }^{12}$ The drug was also well tolerated. Patients in this randomized, placebo-controlled, dose-ranging study were given 2-mg, 5-mg, and 15-mg doses twice daily. At 12 weeks, improvements in PASI 75 response rates were dose-dependent at $25.0 \%$ in the 2 -mg group, $40.8 \%$ in the 5-mg group, and $66.7 \%$ in the 15 -mg group. Phase III trials are currently underway for this oral treatment.

A study of the small-molecule antibiotic STA-21 or ochromycinone that targets the Stat3 transcription factor found that this Stat3 inhibitor improved psoriasis skin lesions. ${ }^{13}$
Psoriatic lesions in 6 out of 8 patients treated with topical ointment containing STA-21 showed improvement after 2 weeks.

A small, double-blind, vehicle-controlled study of the JAK $1 / 2$ inhibitor INCB018424 found that $1 \%$ (once a day) and $1.5 \%$ (twice a day) of this inhibitor applied topically reduced psoriatic lesions, erythema, and scaling in patients with limited plaque psoriasis. ${ }^{14}$ The limitation of this study, aside from its small sample size, was that patients served as their own controls (ie, treating 1 plaque with the active agent in cream formula and 1 plaque with vehicle alone).

Apremilast is a small-molecule phosphodiesterase 4 inhibitor that has shown efficacy in the treatment of moderate to severe plaque psoriasis. A phase IIb, randomized, placebo-controlled, dose-ranging trial investigated the efficacy of apremilast taken orally twice daily at 10,20 , or $30 \mathrm{mg} .{ }^{15}$ Patients were followed over 24 weeks. At week 16 , those in the placebo group were switched to apremilast 20 or $30 \mathrm{mg}$ twice daily. The study found apremilast to be effective, safe, and tolerable in patients with moderate to severe disease. The primary endpoint of PASI 75 at 16 weeks was achieved by significantly more patients on 20and 30-mg doses compared with placebo. ${ }^{16,17}$ Patients on these doses also reported improved QoL outcomes (HRQOL and DLQI) as well as improved pruritus. ${ }^{13}$ The $30-\mathrm{mg}$ dose achieved the best outcome overall and is being studied in phase III trials.

Emerging biologics. IL-17 is thought to be an important treatment target in psoriasis. The efficacy and safety of secukinumab, an anti-IL-17 monoclonal antibody, was studied in the treatment of moderate to severe psoriasis. A phase II, randomized, double-blind, placebo-controlled, dose-ranging study found that $80 \%$ of patients given a dose of $3 \times 150$ mg of secukinumab subcutaneously every 4 weeks achieved a PASI 75 response at 12 weeks. ${ }^{18}$ This study as well as a phase II study concluded that secukinumab exhibited efficacy and was well-tolerated in patients with moderate to severe psoriasis. ${ }^{18,19}$

Brodalumab (AMG 827) is another IL-17 inhibitor that has been investigated in the treatment of psoriasis. A small phase I, randomized, placebo-controlled study of this drug showed that single doses of brodalumab of $350 \mathrm{mg}$ subcutaneously or $700 \mathrm{mg}$ intravenously resulted in a rapid, dosedependent improvement in PASI scores within 2 weeks in patients with moderate to severe disease ${ }^{20}{ }^{2}$ In a phase II, randomized, double-blind, placebo-controlled, dose-ranging study, brodalumab was found to significantly improve psoriasis in patients with moderate to severe disease. ${ }^{21}$ The primary endpoint for the study was the percentage amelioration in PASI scores at 12 weeks. Patients were administered the drug subcutaneously on day 1 and at weeks $1,2,4,6,8$, and 10. Mean percentage improvements increased in a dosedependent fashion: $45 \%$ in patients receiving $70 \mathrm{mg}, 85.9 \%$ in patients receiving $140 \mathrm{mg}, 86.3 \%$ in patients receiving 210 
$\mathrm{mg}$, and $76 \%$ in those receiving $280 \mathrm{mg}$. Two cases of neutropenia were reported in patients who received $210 \mathrm{mg}$.

Ixekizumab, a monoclonal antibody that targets IL-17, was evaluated for safety and efficacy in 142 patients with moderate to severe plaque psoriasis in a double-blind, placebo-controlled trial. ${ }^{22}$ Patients enrolled in the study received $10,25,75$, or $150 \mathrm{mg}$ of ixekizumab subcutaneously at 0,2 , $4,8,12$, and 16 weeks. At week 12, the percentages of patients who experienced a minimum reduction of $75 \%$ in PASI scores were $76.7 \%$ in the $25-\mathrm{mg}$ group, $82.8 \%$ in the $75-\mathrm{mg}$ group, and $82.1 \%$ in the $150-\mathrm{mg}$ group. A reduction in PASI scores by a minimum of $90 \%$ at week 12 was experienced by $50 \%$ of patients in the $25-\mathrm{mg}$ group, $58.6 \%$ in the $75-\mathrm{mg}$ group, and $71.4 \%$ in the $150-\mathrm{mg}$ group. Patients in the 10-mg group did not achieve a significant reduction in PASI scores at week 12 compared with placebo. Adverse events were reported in $63 \%$ of patients in all groups, with nasopharyngitis, upper respiratory infection, injection-site reaction, and headache being the most common incidents.

For more recent updates, see Chapter 17 for a summary of new findings.

\section{Biosimilars}

In 2014, biosimilars for the biologic infliximab gained approval by Health Canada for use in psoriasis care. Health Canada refers to biosimilars as subsequent entry biologics (SEBs). Other biosimilars to existing biologics are currently in various stages of development. ${ }^{23-25}$

Unlike generic drugs, biosimilars are not chemically identical to the original biologic. Biologics are large, complex molecules constructed through recombinant DNA processes expressed in living systems. They are inherently variable and, as such, can never be duplicated exactly. The major benefit of biosimilars to patients and the health care system is the potential cost savings. However, because biosimilars are not identical to the original biologic, their therapeutic efficacy and safety cannot be extrapolated to be the same as the reference biologic drug. Comparative clinical trials with the original biologics are required prior to approval by Health Canada to determine equivalence in terms of efficacy.

\section{References}

1. Nast A, Boehncke WH, Mrowietz U, et al. S3 - Guidelines on the treatment of psoriasis vulgaris (English version): update. J Dtsch Dermatol Ges. 2012;10(suppl 2):S1-S95.

2. Langley RG, Elewski BE, Lebwohl, et al. Secukinumab in plaque psoriasis - results of two phase 3 trials. $N$ Engl J Med. 2014;371(4):326-338.

3. Langley RGB, Feldman SR, Nyirady, et al. The 5-point investigator's global assessment (IGA) scale: a modified tool for evaluating plaque psoriasis severity in clinical trials. $J$ Dermatolog Treat. 2015;26(1):23-31.

4. Gordon KB, Kimball AB, Chau D, et al. Impact of brodalumab treatment on psoriasis symptoms and health-related quality of life: Use of a novel patient-reported outcome measure, the psoriasis symptom inventory. Br J Dermatol. 2014;170(3): 705-715.

5. Revicki DA, Jin Y, Wilson HD, Chau, et al. Reliability and validity of the psoriasis symptom inventory in patients with moderate-to-severe psoriasis. J Dermatol Treat. 2014;25(1): 8-14.

6. Griffiths CE, Strober BE, van de Kerkhof P, et al. Comparison of ustekinumab and etanercept for moderate-to-severe psoriasis. N Engl J Med. 2010;362(2):118-128.

7. Abe R, Yamagishi S, Fujita Y, et al. Topical application of anti-angiogenic peptides based on pigment epithelium-derived factor can improve psoriasis. J Dermatol Sci. 2010;57(3):183191.

8. Crawshaw AA, Griffiths CE, Young HS, et al. Investigational VEGF antagonists for psoriasis. Expert Opin Investig Drugs. 2012;21(1):33-43.

9. Bissonnette R, Bolduc C, Maari C, et al. Efficacy and safety of topically applied $1.0 \%$ WBI-1001 for the treatment of mild to moderate plaque psoriasis. J Eur Acad Derm Venereol. 2012;26:1516-1521.

10. Skvara H, Dawid M, Kleyn E, et al. The PKC inhibitor AEB071 may be a therapeutic option for psoriasis. J Clin Invest. 2008;118(9):3151-3159.

11. Ports WC, Khan S, Lan S, et al. A randomized phase 2a efficacy and safety trial of the topical Janus kinase inhibitor tofacitinib in the treatment of chronic plaque psoriasis. $\mathrm{Br} J$ Dermatol. 2013;169(1):137-145.

12. Papp KA, Menter A, Strober B, et al. Efficacy and safety of tofacitinib, an oral Janus kinase inhibitor, in the treatment of psoriasis: a phase $2 \mathrm{~b}$ randomized placebo-controlled doseranging study. Br J Dermatol. 2012;167(3):668-677.

13. Miyoshi K, Takaishi M, Nakajima K, et al. Stat3 as a therapeutic target for the treatment of psoriasis: a clinical feasibility study with STA-21, a Stat3 inhibitor. J Invest Dermatol. 2011;131(1):108-117.

14. Punwani N, Scherle P, Flores R, et al. Preliminary clinical activity of a topical JAK $1 / 2$ inhibitor in the treatment of psoriasis. J Am Acad Dermatol. 2012;67(4):658-664.

15. Papp K, Cather JC, Rosoph L, et al. Efficacy of apremilast in the treatment of moderate to severe psoriasis: a randomised controlled trial. Lancet. 2012;380(9843):738-746.

16. Papp KA, Kaufmann R, Thaci D, et al. Efficacy and safety of apremilast in subjects with moderate to severe plaque psoriasis: results from a phase II, multicenter, randomized, double-blind, placebo-controlled, parallel-group, dose-comparison study. J Eur Acad Dermatol Venereol. 2013 27(3):376-383.

17. Strand V, Florentino D, Hu C, et al. Improvements in patientreported outcomes with apremilast, an oral phosphodiesterase 4 inhibitor, in the treatment of moderate to severe psoriasis: results from a phase IIb randomized, controlled study. Health Qual Life Outcomes. 2013;11:82.

18. Papp KA, Langley RG, Sigurgeirsson B, et al. Efficacy and safety of secukinumab in the treatment of moderate-tosevere plaque psoriasis: a randomized, double-blind, placebo-controlled phase II dose-ranging study. $\mathrm{Br} J$ Dermatol. 2013;168(2):412-421.

19. Rich P, Sigurgeirsson B, Thaci D, et al. Secukinumab induction and maintenance therapy in moderate-to-severe 
plaque psoriasis: a randomized, double-blind, placebocontrolled, phase II regimen-finding study. Br J Dermatol. 2013;168(2):402-411.

20. Papp KA, Reid C, Foley P, et al. Anti-IL-17 receptor antibody AMG 827 leads to rapid clinical response in subjects with moderate to severe psoriasis: results from a phase I, randomized, placebo-controlled trial. J Invest Dermatol. 2012;132(10):2466-2469.

21. Papp KA, Leonardi C, Menter A, et al. Brodalumab, an antiinterleukin-17-receptor antibody for psoriasis. $N$ Engl J Med. 2012;366(13):1181-1189.

22. Leonardi C, Matheson R, Zachariae, et al. Anti-interleukin-17 monoclonal antibody ixekizumab in chronic plaque psoriasis. N Engl J Med. 2012;366(13):1190-1199.
23. Canadian Dermatology Association. Canadian Dermatology Association Position Statement: Biosimilars. http://www. dermatology.ca/wp-content/uploads/2013/09/BiosimilarsWEB-EN-2013.pdf. Accessed March 2014.

24. Health Canada Health Products and Food Branch. Guidance for sponsors: information and submission requirements for subsequent entry biologics (SEBs). http://www.hc-sc.gc.ca/ $\mathrm{dhp}-\mathrm{mps} / \mathrm{consultation/biolog/submission-seb-exigences-}$ pbu-eng.php. Accessed March 2014.

25. Canadian Agency for Drugs and Technologies in Health (CADTH). Subsequent Entry Biologics - Emerging Trends in Regulatory and Health Technology Assessment Frameworks. http://www.cadth.ca/products/environmental-scanning/environmental-scans/environmental-scan-43. Accessed March 2014. 


\section{Chapter 16: Combination Therapy For Plaque Psoriasis}

Combination therapies are often used in the clinical management of plaque psoriasis. They are commonly used as a strategy to enhance treatment efficacy and reduce the risk of adverse effects due to drug toxicities. Combination therapies are also discussed in Chapter 5 (Management of Mild Plaque Psoriasis) and Chapter 6 (Management of Moderate to Severe Plaque Psoriasis) and in a subsection of Chapter 15 (The Future of Psoriasis Care). There is still a dearth of data on the use of combination therapy for psoriasis, in stark contrast to the many randomized controlled studies available on monotherapies. ${ }^{1}$ However, the number of clinical trials evaluating the efficacy and safety of combination therapy is on the rise. Given the increasing use of combination therapy in practice, this section in the addendum addresses pertinent studies and new clinical data available on these therapies since the publication of the 2009 Canadian Guidelines for the Management of Plaque Psoriasis.

\section{Topical and Systemic Agents}

A systematic review and meta-analysis by Bailey et $\mathrm{al}^{2}$ of combination treatments for psoriasis summarized the evidence for combined topical and systemic therapies (published literature until July 2010). Fifty trials were used in the efficacy analysis. These 50 trials included 66 combination versus monotherapy arms among them, constituting an efficacy analysis of 8325 trial subjects in total. The review concluded that combination therapies were overall more effective than monotherapies.

The literature review also found that adding phototherapy to a topical regimen for psoriasis induced a significant clearance when compared with topical therapy alone. ${ }^{2}$ However, the reverse did not hold true - adding a topical agent to phototherapy did not improve disease severity significantly compared with phototherapy alone.

Two-compound formulations are commonly used in practice as adjunctive therapy to treat resistant lesions in patients with extensive disease who are receiving other therapy (phototherapy, systemic therapy, or biologic agents). ${ }^{3}$ Topical agents can be used to enhance efficacy and diminish disease in patients who are improving but still have active areas of disease. This is a common strategy in the treatment of palmoplantar psoriasis as well-in instances when phototherapy or acitretin are already being used. In these cases, short courses of 2-compound formulations may help boost efficacy.

\section{Vitamin D Derivatives and Corticosteroid Therapy}

The systematic review and meta-analysis by Bailey et $\mathrm{al}^{2}$ concluded that vitamin D derivatives combined with corticosteroid therapy were more effective than vitamin D monotherapy. Another systematic review of the efficacy and safety of firstline topical treatments for chronic plaque psoriasis was performed on available literature until April 2012. ${ }^{4}$ This review, by Hendriks et al, ${ }^{4}$ concluded that the combination of corticosteroids and concurrent vitamin D analogues has had the most extensive investigation. This combination was deemed efficacious (more so than either agent alone) and safe, with 2-compound products providing the most practical solution to combining the 2 agents to treat mild to moderate psoriasis.

Since the publication of the 2009 Guidelines, a 2-compound gel formulation of calcipotriol and betamethasone dipropionate has come on the market for use in treating scalp psoriasis. A 2011 meta-analysis of available RCTs on this gel combination therapy found that it is very effective for moderately severe scalp psoriasis. ${ }^{5}$ The meta-analysis also noted that this combination had a significantly lower risk of adverse events.

Bailey et $\mathrm{al}^{2}$ noted that a corticosteroid combined with hydrocolloid occlusion dressing performed more effectively than corticosteroid therapy alone. Similarly, Hendriks et $\mathrm{al}^{4}$ found that the addition of a hydrogel patch (6-8 hours daily) to a combination of betamethasone dipropionate and calcipotriol to treat psoriasis plaques increased treatment efficacy. A large study included in the Hendriks et al review reported that the addition of hydrocortisone to calcipotriol was more efficacious than the use of calcipotriol alone (Ortonne 2010). The patients using this combination also reported fewer adverse events than those on calcipotriol alone.

A 2010 trial investigated the effects of adding a statin to psoriatic regimens. ${ }^{6}$ This randomized, double-blind, placebo-controlled trial found that the addition of simvastatin to topical betamethasone enhanced its therapeutic effect. Importantly, the cardiovascular protective effect afforded by statin use encourages the use of this drug in psoriasis.

\section{Vitamin D Derivatives Plus Psoralen or Phototherapy}

A study evaluated the safety and efficacy of psoralen gel or calcipotriol ointment in combination with targeted NB-UVB phototherapy. ${ }^{7}$ The study found that psoralen did not improve results compared with phototherapy alone and that calcipotriol ointment enhanced the therapeutic effect of phototherapy in psoriasis. 
Another study investigated combining 308-nm excimer laser with topical treatments for the management of plaque psoriasis. ${ }^{8}$ The study found that laser treatment was safe and effective when combined with calcipotriol. Separate treatments were administered for different plaques within the same patient (this was a within-patient trial).

\section{Vitamin A Derivatives Plus Psoralen, Phototherapy, or Corticosteroid}

In the systematic review by Bailey et $\mathrm{al},{ }^{2}$ the vitamin A derivative acitretin combined with psoralen-UVA (PUVA) and the vitamin A derivative tazarotene combined with corticosteroid were both found to be more effective than using a vitamin A derivative alone. The use of tazarotene combined with corticosteroids to treat mild psoriasis is discussed in Chapter 5 (Management of Mild Plaque Psoriasis). Vitamin A combined with PUVA also appears to be more effective than PUVA monotherapy. Similarly, vitamin A used together with UVB was found to be more effective than UVB alone. ${ }^{2}$ The combination of acitretin and PUVA is discussed in Chapter 6 (Management of Moderate to Severe Plaque Psoriasis) and Chapter 12 (Management of Palmoplantar Psoriasis).

\section{Corticosteroid and Phototherapy}

A study on the use of 308-nm excimer laser combined with topical flumethasone ointment found that this treatment is superior to laser monotherapy. ${ }^{9}$ This randomized, doubleblind study in mild to moderate psoriasis reported that this combination increases efficacy using lower laser doses, which could reduce side effects.

\section{Phototherapy Plus Psoralen or Liquor Carbonis Distillate}

A study comparing NB-UVB alone or combined with 8-methoxypsoralen in treatment-resistant psoriasis plaques on the legs found that the addition of topical methoxsalen afforded no additional benefit. ${ }^{10}$

A 2009 study evaluated the combined use of NB-UVB and topical coal tar formulation composed of $15 \%$ liquor carbonis distillate (LCD) in psoriasis over 12 weeks. ${ }^{11}$ This small, randomized, investigator-blinded, bilateral study compared NB-UVB alone to NB-UVB combined with LCD. The study found that patients receiving NB-UVB and LCD achieved a minimal disease state or disease clearance 3 weeks quicker than those receiving NB-UVB alone.

\section{Combinations to Avoid}

A 2010 literature search that reviewed the benefits and risks associated with available combinations provided some insights to indicate which combinations are useful and which should not be recommended. ${ }^{12}$ The review concluded that salicylic acid should not be combined with vitamin D analogues as it inactivates vitamin $\mathrm{D}$. Three days after stopping salicylic acid appears to constitute a sufficient latency period prior to the use of vitamin D derivatives. Similarly, topical treatments containing salicylic acid should not be used just before UV therapy because salicylic acid absorbs UV and reduces the efficacy of phototherapy as a result. ${ }^{12}$ Salicylic acid should not be combined with cyclosporine, methotrexate, or fumaric acid esters as this may result in nephrotoxic side effects.

A salicylic base improves the stability of anthralin therapy in topical formulation. ${ }^{12}$ It is likely, however, that the use of tar and anthralin will decline further as more cosmetically acceptable alternatives are available. Similarly, the use of cyclosporine will likely decline as newer and safer oral alternatives become available (eg, apremilast).

\section{Systemic and Biologic Agents}

\section{Methotrexate Plus Phototherapy or Etanercept}

A randomized, single-blinded, placebo-controlled trial assessed the efficacy of methotrexate combined with NB-UVB phototherapy versus NB-UVB monotherapy. ${ }^{13}$ The study found that the combination of methotrexate and NB-UVB provided more rapid clinical improvement in psoriasis patients with moderate to severe disease.

A randomized, double-blind, placebo-controlled study evaluated the use of methotrexate combined with etanercept in patients with moderate to severe psoriasis when compared with etanercept alone. ${ }^{14}$ The study concluded that etanercept combined with methotrexate showed an increased efficacy and had an acceptable tolerability profile. Similarly, a randomized, open-label study found that adding etanercept to methotrexate therapy in cases where methotrexate alone does not clear disease has superior efficacy compared with when patients are rotated to an alternate monotherapy (etanercept-methotrexate taper). ${ }^{15}$

\section{Adalimumab and Phototherapy}

In a small, single-arm, open-label study, patients with moderate to severe psoriasis were given adalimumab $40 \mathrm{mg}$ every other week and NB-UVB 3 times a week for 12 weeks and were observed for an additional 12 weeks without any treatment. ${ }^{16}$ Improvement was observed until the end of 24 weeks, and no serious adverse events were reported. The study concluded that adalimumab used in combination with NB-UVB was clinically effective and well tolerated. These findings aligned well with historical data that include results from the randomized controlled evaluation of adalimumab every other week dosing in moderate to severe psoriasis trial (REVEAL) study (phase III trial of adalimumab) as well as the utilization of narrow-band ultraviolet light B therapy and etanercept for the treatment of psoriasis (UNITE) study (open-label study of NB-UVB plus etanercept).

\section{Etanercept Plus Acitretin or Phototherapy}

A randomized, controlled, investigator-blinded pilot trial evaluated the efficacy and safety of acitretin combined with 
etanercept in moderate to severe psoriasis. ${ }^{17}$ The 2008 study was the first to investigate the use of a biologic agent combined with a conventional agent. The study found that a regimen of etanercept $25 \mathrm{mg}$ once weekly and acitretin $0.4 \mathrm{mg} \mathrm{kg}^{-1}$ daily over 24 weeks was as effective as etanercept $25 \mathrm{mg}$ twice weekly and more effective than acitretin alone. ${ }^{17}$ Patients using a biologic agent as well as acitretin in combination may achieve disease control with lower amounts of either agent. Thus, the addition of acitretin to a treatment regimen offers the potential advantage of a reduction in the risk of cancer without sacrificing efficacy. Larger studies are needed to confirm these results.

The concurrent use of biologic agents and NB-UVB in patients with moderate to severe psoriasis has been investigated in several biologic agents, including etanercept. A 2011 prospective study investigated the use of NB-UVB with etanercept compared with etanercept monotherapy. The results indicated that etanercept ( $25 \mathrm{mg}$ twice weekly) combined with NB-UVB phototherapy ( 3 times weekly) was more effective than etanercept alone after 6 weeks of treatment in patients with moderate to severe disease. ${ }^{18} \mathrm{An}$ increased risk of skin malignancy is associated with TNF- $\alpha$ inhibitors as well as phototherapy; thus, the investigators recommended that use of this combination be restricted to short periods of use in instances when a quick initial response is desired or a flare needs to be controlled. Long-term treatment needs to be avoided.

A 2012 randomized, single-blinded study investigated this combination in patients with moderate to severe psoriasis who failed to achieve PASI 90 after 12 weeks of etanercept monotherapy (50 mg once a week). ${ }^{19}$ The study found that in patients with high adherence rates to NB-UVB phototherapy, PASI 90 was achieved by $16.2 \%$ of patients receiving combination therapy versus $3.4 \%$ of those receiving etanercept alone. The study concluded that the addition of NB-UVB ( 3 times weekly) after 12 weeks of etanercept did not significantly improve clinical response in patients except for an improved response at weeks 16 and 24 in a small subset with high adherence to phototherapy. Poor adherence was the main limitation in this study.

In terms of potential risks, a small study assessed the impact of etanercept on inflammation due to UVB exposure, cell cycle regulation, and DNA damage. ${ }^{20}$ The study results indicated that treatment with broadband UVB and a TNF- $\alpha$ inhibitor may increase the risk of photocarcinogenesis.

An 2010 article reviewing the clinical evidence on combining etanercept with various traditional agents in patients with moderate to severe disease found that etanercept may be useful in maintaining disease control established with cyclosporine. ${ }^{21}$ However, this was based on a string of case studies, and larger studies would be needed to confirm this result.

\section{Corticosteroid Spray and Biologic Therapy}

An open-label phase IV study evaluated the efficacy of a potent corticosteroid spray (clobetasol propionate $0.05 \%$ ) used concomitantly with systemic biologic therapy in moderate to severe psoriasis. ${ }^{22}$ Patients already receiving a biologic treatment regimen used the spray on lesions twice daily for 4 weeks. target plaque severity (TPS) was evaluated at baseline and again at the end of 4 weeks. The results showed that $81 \%$ of patients with moderate TPS, $79.5 \%$ of patients with severe TPS, and $58.8 \%$ of patients with very severe TPS at baseline were rated as "clear" or "almost" clear after 4 weeks. The addition of this spray to biologic therapy was deemed well tolerated. Further studies are needed to confirm the efficacy and safety of this therapy. The corticosteroid spray could present a treatment option for psoriasis in future. An earlier (2007) large, open-label study that evaluated the use of this spray as an addon to other stable treatments also found the spray to be effective and well tolerated. ${ }^{23}$

\section{Recommendations}

Recommendation and Level of Evidence

For appropriate patients, phototherapy alone or combined with approved topical therapy for psoriasis may be used (Ref. 2, LoE I++).

Use of excimer laser combined with calcipotriol can be considered in the treatment of localized plaques (Ref. 8, LoE 2+).

For appropriate patients, acitretin combined with PUVA can be used as treatment (Ref. 2, LoE I++).

For appropriate patients, tazarotene combined with a topical corticosteroid can be used as treatment (Ref. 2, LoE I++).

Salicylic acid should not be combined with vitamin $D$ derivatives or used just prior to phototherapy in the treatment of psoriasis (Ref. 12, LoE 2++).

Salicylic acid should not be used in combination with cyclosporine, methotrexate, or fumaric acid esters in the treatment of psoriasis (Ref. 12, LoE 2++).

Methotrexate can be added to NB-UVB phototherapy for the treatment of moderate to severe psoriasis (Ref. 13, LoE 2+).

Methotrexate can be added to etanercept therapy for patients with moderate to severe psoriasis (Refs. 14, 15, LoE 2+).

For patients with moderate to severe psoriasis, etanercept or adalimumab in combination with NBUVB can be used for short-term treatment. Phototherapy should be used for a limited period of time due to the possible increased risk of cutaneous malignancy (Refs. 16, 18, LoE 2+).
Grade of

Recommendation

Grade A

Grade C

Grade A

Grade A

Grade B

Grade D

Grade C

Grade C

Grade C 


\section{References}

1. Gustafson CJ, Watkins C, Hix E, et al. Combination therapy in psoriasis: an evidence-based review. Am J Clin Dermatol. 2013;14(1):9-25.

2. Bailey EE, Ference EH, Alikhan A, et al. Combination treatments for psoriasis: a systematic review and meta-analysis. Arch Dermatol. 2012;148(4):511-522.

3. Saraceno R, Gramiccia T, Frascione P, et al. Calcipotriene/ betamethasone in the treatment of psoriasis: a review article. Expert Opin Pharmacother. 2009;10(14):2357-2365.

4. Hendriks AG, Keijsers RR, de Jong EM, et al. Efficacy and safety of combinations of first-line topical treatments in chronic plaque psoriasis: a systematic literature review. J Eur Acad Dermatol Venereol. 2013;27(8):931-951.

5. Bottomley JM, Taylor RS, Ryttov J, et al. The effectiveness of two-compound formulation calcipotriol and betamethasone dipropionate gel in the treatment of moderately severe scalp psoriasis: a systematic review of direct and indirect evidence. Curr Med Res Opin. 2011;27(1):251-268.

6. Naseri M, Hadipour A, Sepaskhah, et al. The remarkable beneficial effect of adding oral simvastatin to topical betamethasone for treatment of psoriasis: a double-blind, randomized, placebo-controlled study. Niger J Med. 2010;19(1):58-61.

7. Ozkan I, Kose O, Ozmen I, et al. Efficacy and safety of nonlaser, targeted UVB phototherapy alone and in combination with psoralen gel or calcipotriol ointment in the treatment of localized, chronic, plaque-type psoriasis. Int J Dermatol. 2012;51(5):609-613.

8. Rogalski C, Grunewald S, Schedtschorke M, et al. Treatment of plaque-type psoriasis with the $308 \mathrm{~nm}$ excimer laser in combination with dithranol or calcipotriol. Int $J$ Hyperthermia. 2012;28(2):184-190.

9. Dong J, He Y, Zhang X, et al. Clinical efficacy of flumetasone/ salicylic acid ointment combined with 308-nm excimer laser for treatment of psoriasis vulgaris. Photodermatol Photoimmunol Photomed. 2012;28(3):133-136.

10. Ehsani AH, Ghaninejad H, Kiani A, et al. Comparison of topical 8-methoxypsoralen and narrowband ultraviolet B with narrowband ultraviolet B alone in treatment-resistant sites in plaquetype psoriasis: a placebo-controlled study. Photodermatol Photoimmunol Photomed. 2011;27(6):294-296.

11. Bagel J. LCD plus NB-UVB reduces time to improvement of psoriasis vs. NB-UVB alone. J Drugs Dermatol. 2009;8(4):351-357.
12. Domm S and Mrowietz U. Combination therapy in the treatment of psoriasis. J Dtsch Dermatol Ges. 2011;9(2):94-98.

13. Mahajan R, Kaur I and Kanwar AJ. Methotrexate/narrowband UVB phototherapy combination vs. narrowband UVB phototherapy in the treatment of chronic plaque-type psoriasis-a randomized single-blinded placebo-controlled study. $J$ Eur Acad Dermatol Venereol. 2010;24(5):595-600.

14. Gottlieb AB, Langley RG, Strober BE, et al. A randomized, double-blind, placebo-controlled study to evaluate the addition of methotrexate to etanercept in patients with moderate to severe plaque psoriasis. Br J Dermatol. 2012;167(3):649-657.

15. Zachariae C, Mork NJ, Reunala T, et al. The combination of etanercept and methotrexate increases the effectiveness of treatment in active psoriasis despite inadequate effect of methotrexate therapy. Acta Derm Venereol. 2008;88(5):495-501.

16. Bagel J. Adalimumab plus narrowband ultraviolet B light phototherapy for the treatment of moderate to severe psoriasis. J Drugs Dermatol. 2011;10(4):366-371.

17. Gisondi P, Del Giglio M, Cotena C, et al. Combining etanercept and acitretin in the therapy of chronic plaque psoriasis: a 24-week, randomized, controlled, investigator-blinded pilot trial. Br J Dermatol. 2008;158(6):1345-1349.

18. Gambichler T, Tigges C, Scola N, et al. Etanercept plus narrowband ultraviolet $\mathrm{B}$ phototherapy of psoriasis is more effective than etanercept monotherapy at 6 weeks. Br J Dermatol. 2011;164(6):1383-1386.

19. Lynde CW, Gupta AK, Guenther L, et al. A randomized study comparing the combination of nbUVB and etanercept to etanercept monotherapy in patients with psoriasis who do not exhibit an excellent response after 12 weeks of etanercept. J Dermatolog Treat. 2012;23(4):261-267.

20. Gambichler T, Tigges C, Dith A, et al. Impact of etanercept treatment on ultraviolet B-induced inflammation, cell cycle regulation and DNA damage. Br J Dermatol. 2011;164(1):110-115.

21. Foley PA, Quirk C, Sullivan JR, et al. Combining etanercept with traditional agents in the treatment of psoriasis: a review of the clinical evidence. J Eur Acad Dermatol Venereol. 2010;24(10):1135-1143.

22. Feldman SR, Koo JY, Johnson LA, et al. Clobetasol propionate spray $0.05 \%$ add-on therapy to a stable regimen of biologic treatment in patients with moderate to very severe plaque psoriasis. Cutis. 2009;84(4 suppl):25-32.

23. Feldman SR. Effectiveness of clobetasol propionate spray $0.05 \%$ added to other stable treatments: add-on therapy in the COBRA trial. Cutis. 2007;80(5 suppl):20-28. 


\section{Chapter I7: Summary of New Findings}

This chapter summarizes pertinent papers in the literature on plaque psoriasis published from January 2014 to December 2015.

\section{Moderate to Severe Psoriasis}

An observational surveillance registry examined the longterm safety and efficacy of etanercept in psoriasis. ${ }^{1}$ The OBSERVE-5 surveillance registry recorded the adverse events for 2510 patients with moderate to severe psoriasis over 5 years. The cumulative incidence was $22.2 \%$ for serious adverse events, $6.5 \%$ for serious infections, $3.2 \%$ for malignancies (excluding nonmelanoma skin cancer), 3.6\% for nonmelanoma skin cancer, $2.8 \%$ for coronary artery disease, $0.7 \%$ for psoriasis worsening, $0.2 \%$ for central nervous system demyelinating disorder, and $0.1 \%$ each for lymphoma, tuberculosis, opportunistic infections, and lupus, respectively. No new safety signals were observed with longterm use of etanercept. The study authors noted that one limitation of the study was that no comparator group was included.

In a multicentre cohort study, the extent of antidrug antibody (ADA) formation in 80 patients receiving adalimumab was evaluated in a 1-year follow-up. ${ }^{2}$ Adalimumab and ADA concentrations were assessed at baseline and at 12, 24, and 52 weeks. A highly drug-tolerant assay revealed ADA formation in $90 \%$ of patients before week 24 and in $49 \%$ of patients at the end of 52 weeks. The study found that patients with no ADA formation in the first 24 weeks of treatment had a small chance of ADA formation in the next 24 weeks. The presence of ADA strongly correlated with adalimumab concentration (correlation coefficient of 0.519 ). The study noted that in the presence of ADA, dose interval shortening was less useful.

\section{Topical Therapy}

In a phase II multicentre trial of 376 patients with psoriasis, the efficacy of an aerosol foam formulation of a fixed combination of calcipotriol $(0.005 \%)$ and betamethasone $(0.064 \%)$ was compared with that of an ointment formula. ${ }^{3}$ The scalp was not treated in this study. At the end of 4 weeks, $54.6 \%$ of patients using the foam formulation achieved a "clear/almost clear" PGA score compared with $43 \%$ of those using the ointment. The study noted that the calcipotriol/betamethasone aerosol foam formula showed greater efficacy and similar tolerability compared with the ointment. Another multicentre, single-arm, open-label study evaluated the systemic safety of the calcipotriol/betamethasone dipropionate aerosol foam in 35 patients over 4 weeks. ${ }^{4}$ The study found that at maximal use (calcipotriol $50 \mu \mathrm{g} / \mathrm{g}$ plus betamethasone $0.5 \mathrm{mg} / \mathrm{g}$ applied once daily) in moderate to severe psoriasis, adrenocorticotropic hormone responses were normal and changes in calcium homeostasis were not clinically relevant.

\section{Special Populations and Circumstances}

\section{Psoriasis in Children}

In a small phase III study, the safety and efficacy of ustekinumab were assessed in 110 adolescents aged 12 to 17 years with moderate to severe plaque psoriasis. ${ }^{5}$ Patients were randomized to receive standard or half-dosing of ustekinumab or placebo. At 12 weeks, $67.6 \%$ of patients receiving the half dose of ustekinumab and $69.4 \%$ of patients receiving the standard dose achieved a PGA rating of "clear/almost clear" compared with $5.4 \%$ of those receiving placebo. PASI 75 was achieved by $78.4 \%$ of patients in the half-dose group and $80.6 \%$ of patients in the standard dosing group compared with $10.8 \%$ for those receiving placebo. PASI 90 was achieved by $54.1 \%$ of patients in the half-dose group and $61.1 \%$ of patients in the standard dosing group compared with $5.4 \%$ for those receiving placebo. The study concluded that the standard ustekinumab dose provided a comparable response to that in adults. No unexpected adverse events were reported in either group throughout the 52 weeks.

A phase II, multicentre, open-label study investigated the safety over 8 weeks of a once-daily application of a fixeddose combination of calcipotriol/betamethasone dipropionate gel in 78 adolescents. ${ }^{6}$ Patients were aged 12 to 17 years and had moderate to severe scalp psoriasis. At 8 weeks, 28 patients reported adverse events but none of these were serious adverse events. At the end of 8 weeks, 66 patients (85\%) were rated "clear/almost clear" according to the IGA. The study concluded that the gel formulation of calcipotriol/betamethasone dipropionate was well tolerated and effective for scalp psoriasis in adolescents.

A study evaluated the efficacy and safety of calcipotriol/ betamethasone dipropionate for scalp psoriasis in children. Data were extracted from a prospective observational daily clinical practice registry. ${ }^{7}$ Of the 84 treatment episodes analysed, significant improvement of psoriasis was observed in the first 12 weeks using the Psoriasis Scalp Severity Index (PSSI), and the result was maintained over 48 weeks. A prospective study of 73 children with psoriasis that culled data from a pediatric psoriasis registry found that calcipotriol/ betamethasone dipropionate ointment improved mild to moderate psoriasis at 12 weeks and maintained this result for a median time of 35 weeks. ${ }^{8}$ 


\section{Pregnancy}

In a review of the safety and efficacy of psoriasis treatments in pregnancy, an overview of the evidence on pregnancy outcomes in psoriasis patients and the safety of the systemic and topical treatments available was provided. ${ }^{9}$ Key findings of the review were that local corticosteroid and UVB light therapy (UVB and NB-UVB) can be used during pregnancy and that cyclosporine or TNF- $\alpha$ inhibitors should be considered in severe cases.

\section{Patients With Hepatitis B}

A multicentre study of 20 psoriasis patients was performed to investigate the risk of hepatitis B virus (HBV) reactivation in patients receiving biologic therapy for psoriasis. ${ }^{10}$ Inclusion criteria for patients were prior treatment with an anti-TNF or ustekinumab, serological evidence of past HBV infection, absence of hepatitis B surface antigen, and at least one HBV DNA assessment while receiving biologic therapy. After a median follow-up period of 40 months, none of the patients in the study exhibited HBV reactivation. Combining the data from this study with other studies on patients with HBV history, the authors calculated a maximum estimated risk of HBV reactivation of 2.7 reactivations per 100 patient-years over a mean follow-up period of 30 months. The study stressed the importance of measuring viral load in these patients prior to initiation of biologic therapy and regular monitoring by a hepatologist.

\section{Flexural Psoriasis}

A systematic review was conducted to investigate whether flexural psoriasis is a separate disease entity or a variant of plaque psoriasis. ${ }^{11}$ The review investigated current literature on the epidemiology, pathogenesis, clinical and histological presentation, microbiology, and treatment aspects of flexural psoriasis. The authors concluded that no separate pathogenic mechanism was found for flexural psoriasis. No sustainable proof for a bacterial or fungal role in the disease or any correlation of the disease to human leucocyte antigen was found. Although the authors found no evidence to support a different disease entity, they noted the dearth of literature on flexural psoriasis and suggested that it is an underestimated problem.

\section{Nail and Scalp Psoriasis}

The Medical Board for the National Psoriasis Foundation performed a literature review of all nail psoriasis treatments published from January 1947 to May 2014 in an effort to develop best practice recommendations. ${ }^{12}$ The authors concluded that for disease limited to the nails, initial options should include high-potency topical corticosteroids with or without calcipotriol. When topical therapy fails for patients with significant nail disease, treatment with adalimumab, etanercept, intralesional corticosteroids, ustekinumab, methotrexate, or acitretin was recommended by the authors. Adalimumab, etanercept, and ustekinumab were strongly recommended, while methotrexate, acitretin, infliximab, and apremilast were also recommended for patients with significant skin and nail disease. In patients with significant nail, skin, and joint disease, adalimumab, etanercept, ustekinumab, infliximab, methotrexate, apremilast, and golimumab were recommended by the authors.

Cutaneous severity using PASI and NAPSI was assessed from January 2012 to March 2013 in a cross-sectional study of 65 patients. ${ }^{13}$ The prevalence of nail psoriasis was reported at $46.1 \%$; onycholysis was reported in $80 \%$ of patients, making this the most common feature in patients with nail psoriasis. These patients also had a lower mean age, longer disease duration, higher PASI, higher frequency of PsA, and a family history of nail involvement.

In an evaluation of the efficacy of apremilast in nail and scalp psoriasis in 2 phase III RCTs (Efficacy and Safety Trial Evaluating the Effects of Apremilast in Psoriasis [ESTEEM] 1 and 2), 1255 patients received apremilast $30 \mathrm{mg}$ or placebo twice daily. ${ }^{14}$ At week 16, placebo patients were switched to apremilast until week 32, which was followed by a randomized withdrawal to week 52. In ESTEEM 1 and ESTEEM 2, respectively, $66.1 \%$ and $64.7 \%$ of patients had nail psoriasis whereas $66.7 \%$ and $65.5 \%$ of patients had moderate to severe scalp psoriasis at baseline. At week 16, apremilast treatment showed greater improvement in NAPSI, NAPSI-50, and Scalp PGA scores compared with placebo. Improvement was sustained over 52 weeks in patients with PASI response at week 32.

In a phase II study of ixekizumab in patients with chronic plaque psoriasis, the changes in nail and scalp psoriasis were evaluated in 142 patients with moderate to severe psoriasis. ${ }^{15}$ Of the 142 patients, 58 had nail psoriasis and 105 had scalp psoriasis at baseline. Patients received placebo or 10, 25, 75, or $150 \mathrm{mg}$ of ixekizumab. After 20 weeks, significant mean change and improvement from baseline were observed in all groups except the 10-mg group compared with placebo. In the 48-week open-label extension, all patients were given $120 \mathrm{mg}$ of ixekizumab every 4 weeks. At the end of 48 weeks, $78 \%$ of patients with scalp psoriasis and $51 \%$ of patients with nail psoriasis exhibited complete resolution of lesions. Patients were evaluated using NAPSI and PSSI.

A phase IV, multicentre RCT prospective study in 1795 patients with mild to moderate psoriasis evaluated the efficacy of a fixed combination of calcipotriol/betamethasone dipropionate gel. ${ }^{16}$ Patients who were unsuccessfully treated with topical psoriasis treatment 8 weeks prior were followed over a 56-week maintenance period. The PGA, Patient's Self Global Assessment, and Patient Preference Questionnaire (PPQ) were used to assess preference in patients and physicians. After 8 weeks of treatment with the gel, $36.5 \%$ of physicians rated patient psoriasis as "clear/almost clear" whereas $34.2 \%$ patients rated their psoriasis as "clear/almost clear." According to the PPQ results recorded, the majority of patients judged their 8-week treatment with the gel to be preferable over other previous topical treatments. The multicentre, prospective, observational patient reported outcomes 
in a long-term study (PRO-long) also captured patients' perspectives and found that greater treatment satisfaction, ease of use, faster application, and more convenience were reported for the gel format compared with the ointment. ${ }^{17}$

\section{Palmoplantar Psoriasis}

In a retrospective, nonrandomized cohort study of safety and efficacy in 248 patients with plaque-type palmoplantar psoriasis treated at a phototherapy centre, broadband UVB was compared with PUVA treatment. ${ }^{18}$ The study found that of the 122 patients treated with broadband UVB and 126 who received PUVA, 36 patients and 53 patients experienced complete remission, respectively ( 29 and 59 partial responders, respectively). The study concluded that broadband and PUVA are good treatment options with PUVA showing a better and more extended response.

In a review evaluating the use of methotrexate alone or in combination with other systemic therapies in 48 patients with palmoplantar psoriasis, methotrexate was deemed an effective therapy as monotherapy or in combination with other systemic agents. ${ }^{19}$

\section{Social and Psychological Aspects of Psoriasis}

In a systematic review of RCTs on the effects of biologics on depressive symptoms in psoriasis patients with moderate to severe disease, adalimumab, etanercept, and ustekinumab were associated with statistically significant reductions in depressive symptom scores measured on various scales. ${ }^{20}$ Of the 305 publications identified, 3 met the inclusion criteria. In a trial of ustekinumab, mean change in Hospital and Anxiety Depression Rating Scale at 24 weeks was 3.1 versus 0.21 with placebo. In a trial of adalimumab, the mean change in the Zung Self-Rating Depression Scale at 12 weeks was -6.7 versus -1.5 with placebo. Finally, in a trial on etanercept, the between-group difference in the Beck Depression Inventory Scale at 12 weeks was 1.8 in favour of etanercept over placebo.

A multicentre, observational study in 354 patients evaluated the association of genital psoriasis with sexual functioning and its impact on QoL. ${ }^{21}$ Of the 354 patients in the study, 134 had current genital psoriasis disease involvement and 224 had a current and/or previous history of genital involvement. The study found that $87 \%$ of patients reported itch, $39 \%$ reported pain, and $42 \%$ reported dyspareunia. In relation to sexual functioning, $43 \%$ of patients reported a decrease in frequency of intercourse while $32 \%$ reported a worsening of genital psoriasis following intercourse. Using the DLQI, the Centre for Epidemiological Studies Depression Scale, and the Relationship and Sexual Scale, the study confirmed that patients with genital involvement experienced more impairment in sexual health and QoL compared with psoriasis patients without genital involvement. No association was found with circumcision or obesity, although younger age of onset of psoriasis, male sex, and severe disease, as well as scalp, flexural, and nail involvement, were associated with genital psoriasis.

\section{Comorbidities}

A large cohort study examined the association between psoriasis and the risk of major cardiovascular events, including MI, acute coronary syndrome, unstable angina, and stroke. ${ }^{22}$ The study reported the incidences of these cardiovascular events over 5.2 years in 48,523 psoriasis patients and 208,187 controls. The study found that 1257 $(2.59 \%)$ patients with psoriasis had a major cardiovascular event compared with 4784 (2.3\%) controls. The HRs of inflammatory arthritis (HR 1.36), diabetes (HR 1.18), CKD (HR 1.18), hypertension (HR 1.37), transient ischemic attack (HR 2.74), atrial fibrillation (HR 1.54), valvular heart disease (HR 1.23), thromboembolism (HR 1.32), congestive heart failure (HR 1.57), depression (HR 1.16), current smoker (HR 2.18), age (HR 1.07), and male gender (HR 1.83) were also reported in the multivariable analysis. These variables were statistically significant for the risk of major cardiovascular events. The HRs adjusted for age and gender of a major cardiovascular event for psoriasis were 1.10 and 1.40 for severe psoriasis. After adjustment for known CVD risk factors, psoriasis, and severe psoriasis were not found to be associated with the risk of major cardiovascular events in the short to medium term (3-5 years).

A study sought to determine the risk of CKD in psoriasis patients and the impact of medications, psoriasis severity, and comorbidities on the risk of glomerulonephritis in psoriasis patients. ${ }^{23}$ The cohort study enrolled 4344 patients with psoriasis and 13,032 control subjects. All subjects were followed for a 5-year period, and subjects who developed glomerulonephritis or CKD were identified. Psoriasis was found to be independently associated with CKD after adjustment for traditional CKD risk factors (HR 1.28). The study suggests that the increased incidence of glomerulonephritis in psoriasis patients may partly contribute to the association between psoriasis and CKD. Regardless of disease severity, all psoriasis patients exhibited an increased risk for CKD and glomerulonephritis, although risk increased with severity. Patients with arthritis also had a higher risk for CKD (HR 1.62) than did those without (HR 1.26). Among the medications evaluated in psoriasis patients, NSAIDs showed the strongest association with CKD.

\section{Future of Psoriasis Care}

Two phase III studies of patients with moderate to severe psoriasis compared the efficacy of brodalumab $(210 \mathrm{mg}$ or 140 mg every 2 weeks) with that of ustekinumab (45 mg or $90 \mathrm{mg}$ according to body weight) over 12 weeks. ${ }^{24}$ A Phase 3 Study to Evaluate the Efficacy and Safety of Induction and Maintenance Regimens of Brodalumab Compared With Placebo and Ustekinumab in Subjects With Moderate to 


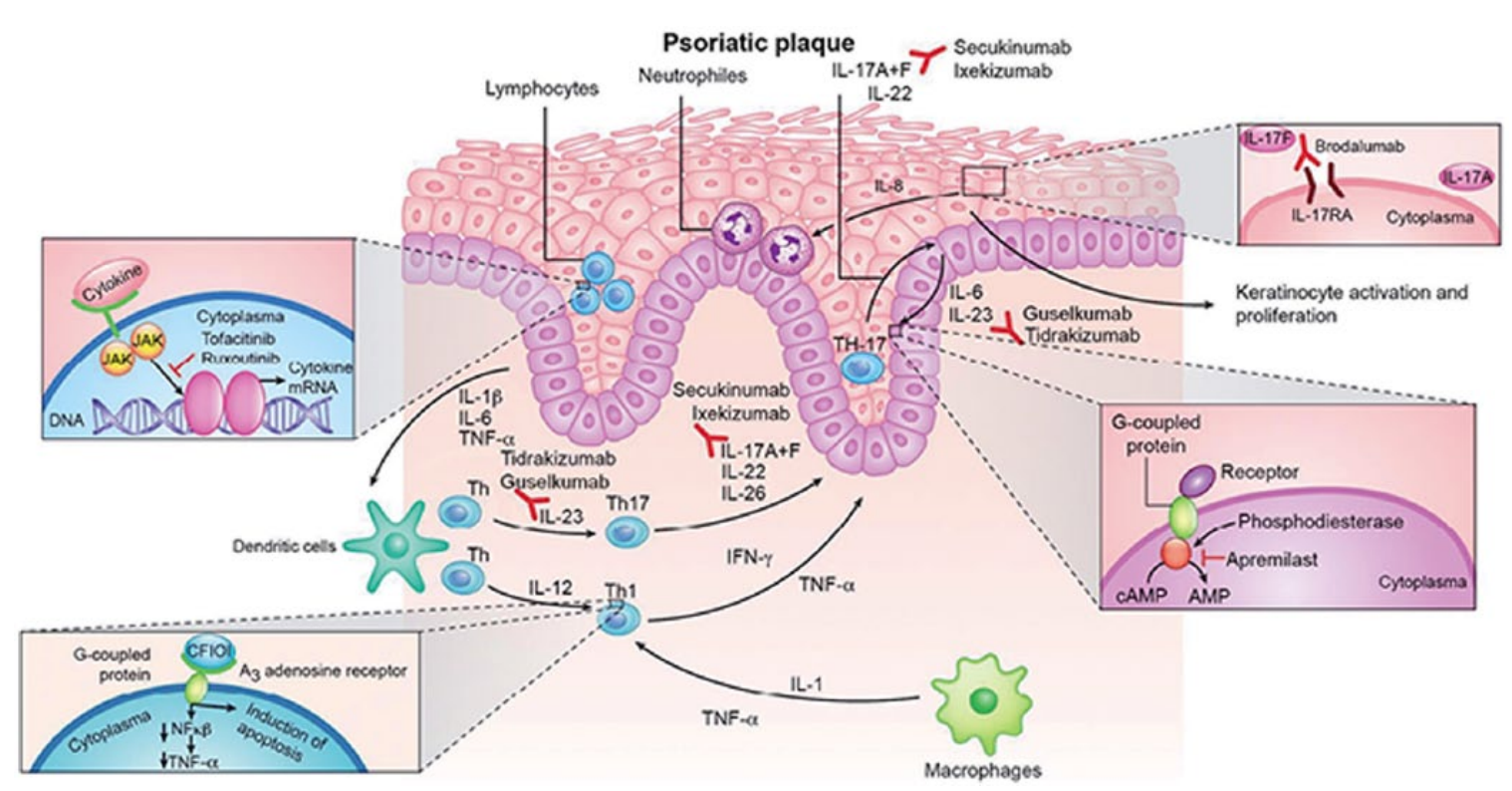

Figure I. Targets of new biologics and small molecules in psoriasis.

New biologics and small molecules under development for the treatment of plaque psoriasis. Dendritic cells produce IL-I2 and IL-23, implicated in ThI and ThI 7 lymphocyte differentiation, respectively. IL-I, produced by activated macrophages is an important mediator of the inflammatory response. TNF- $\alpha$, IL-I7, IL-22, and IL-26 stimulate keratinocyte production of cytokines, chemokines, and adhesion molecules, which, in turn, attract neutrophils and T lymphocytes, leading to amplification of inflammation. Blocking targets of the new biologics are IL-23 (tildrakizumab and guselkumab), IL-I7 (secukinumab and ixekizumab), and IL-I7 receptor (brodalumab). Blocking targets of the small molecules are phosphodiesterase 4 (apremilast) and JAK (tofacitinib and ruxolitinib). Source: Adapted from Kofoed K, Skov L, Zachariae C. New drugs and treatment targets in psoriasis. Acta Derm Venereol. 20I 4;95(2):I33-I39.

Severe Plaque Psoriasis: AMAGINE-2 (1831 patients) and AMAGINE-3 (1881 patients) sought to evaluate the efficacy of brodalumab compared with placebo over 12 weeks using the PASI 75 and a static PGA (sPGA) score of 0 or 1 ("clear/ almost clear" skin). In addition, efficacy of brodalumab was compared with that of ustekinumab using PASI 100. At week 12 , the PASI 75 response rates were higher with brodalumab at the 210-mg and 140-mg doses compared with placebo- $86 \%$ and $67 \%$ versus $8 \%$ in AMAGINE- 2 and $85 \%$ and $69 \%$ versus $6 \%$ in AMAGINE-3 - as were the sPGA scores. Also, at week 12 , the PASI 100 response rates were significantly higher for brodalumab $210 \mathrm{mg}$ than for ustekinumab (44\% vs $22 \%$ in AMAGINE-2 and $37 \%$ vs $19 \%$ in AMAGINE-3).

A 52-week open-label study of ixekizumab evaluated the long-term efficacy and safety of the drug using PASI 75 as a primary endpoint. ${ }^{25}$ The patients enrolled in the open-label extension were drawn from the phase II study of ixekizumab. In all, 120 patients with less than $75 \%$ improvement from baseline on the PASI score were entered into the study after receiving 10 , 25,75 , or $150 \mathrm{mg}$ of ixekizumab or placebo during the phase II trial. Patients with PASI 75 scores or higher were entered into a treatment-free period and enrolled in the extension study after meeting the response criteria. During the extension study, all patients received $120 \mathrm{mg}$ of ixekizumab every 4 weeks. Of the 120 enrolled, 103 completed the 52 weeks of treatment, $77 \%$ of whom achieved PASI 75 at week 52. Irrespective of dose received during the phase II study, all patients in the extension study had similar response rates at week 52 .
Two phase III double-blind RCTs comparing ixekizumab with etanercept or placebo in moderate to severe psoriasis patients evaluated the sPGA scores and PASI 75 scores in 1224 patients (A 12-Week Multicenter, Randomized, Double-Blind, Placebo-Controlled Study Comparing the Efficacy and Safety of LY2439821 to Etanercept and Placebo in Patients With Moderate to Severe Plaque Psoriasis [UNCOVER-2]) and 1346 patients (A 12-Week Multicenter, Randomized, Double-Blind, Placebo-Controlled Study Comparing the Efficacy and Safety of LY2439821 to Etanercept and Placebo in Patients With Moderate to Severe Plaque Psoriasis With a Long-Term Extension Period [UNCOVER 3]). ${ }^{26}$ Patients received 1 injection of $80 \mathrm{mg}$ ixekizumab every 2 weeks or every 4 weeks following a 160mg starting dose; or etanercept $50 \mathrm{mg}$ twice weekly; or subcutaneous placebo. At 12 weeks, $89.7 \%$ (UNCOVER-2) and $87.3 \%$ (UNCOVER-3) of the ixekizumab group receiving 80 mg every 2 weeks achieved a PASI 75 clearance. In the group receiving ixekizumab $80 \mathrm{mg}$ every 4 weeks, $77.5 \%$ (UNCOVER-2) and 84.2\% (UNCOVER-3) achieved PASI 75. This can be compared with $2.4 \%$ (UNCOVER-2) and $7.3 \%$ (UNCOVER-3) of the placebo group and $41.6 \%$ (UNCOVER-2) and 53.4\% (UNCOVER-3) of the etanercept group who achieved PASI 75 at the end of 12 weeks. In the group receiving ixekizumab every 2 weeks, an sPGA score of "clear/almost clear" was achieved by $83.2 \%$ (UNCOVER-2) and $80.5 \%$ (UNCOVER-3) of patients at the end of 12 weeks compared with $2.4 \%$ (UNCOVER-2) and 
$6.7 \%$ (UNCOVER-3) of the placebo group and $36 \%$ (UNCOVER-2) and 41.6\% (UNCOVER-3) of the etanercept group. In the group receiving ixekizumab every 4 weeks, an sPGA score of "clear/almost clear" was achieved by $72.9 \%$ (UNCOVER-2) and 75.4\% (UNCOVER-3) of patients. The study concluded that both ixekizumab regimes showed greater efficacy than placebo and etanercept over 12 weeks.

In 2 phase III double-blind studies of secukinumab, the efficacy and safety of 2 fixed doses of secukinumab (Efficacy of Response and Safety of Two Fixed Secukinumab Regimens in Psoriasis [ERASURE]) and a comparison with etanercept using 2 dosing regimens of secukinumab (Full Year Investigative Examination of Secukinumab vs. Etanercept Using Two Dosing Regimens to Determine Efficacy in Psoriasis [FIXTURE]) were evaluated. ${ }^{27}$ In the studies, 738 patients (ERASURE) and 1306 patients (FIXTURE) were given placebo or doses of $300 \mathrm{mg}$ or $150 \mathrm{mg}$ of secukinumab once weekly for 5 weeks and then every 4 weeks or (for FIXTURE only) $50 \mathrm{mg}$ of etanercept twice weekly for 12 weeks and then once weekly. PASI 75 and a "clear/almost clear" score on a 5-point modified investigator's global assessment (IGA) were the primary endpoints. At 12 weeks, $81.6 \%$ of patients in the $300-\mathrm{mg}$ group and $71.6 \%$ of patients in the 150-mg group receiving secukinumab met the criterion for PASI 75 compared with $4.5 \%$ with placebo in the ERASURE study. For the FIXTURE study, $77.1 \%$ of the 300 $\mathrm{mg}$ and $67 \%$ of the $150-\mathrm{mg}$ groups receiving secukinumab met the PASI 75 criterion compared with $44 \%$ for the etanercept group and $4.9 \%$ for the placebo group. At week 12, $65.3 \%$ of the $300-\mathrm{mg}$ group and $51.2 \%$ of the $150-\mathrm{mg}$ group receiving secukinumab scored "clear/almost clear" on the modified IGA compared with $2.4 \%$ in the placebo group in the ERASURE study. These rates were $62.5 \%$ for the $300-\mathrm{mg}$ and $51.1 \%$ for the 150 -mg groups receiving secukinumab compared with $27.2 \%$ for the etanercept group and $2.8 \%$ for the placebo group in the FIXTURE study.

In a phase II dose-ranging, active-comparator study of guselkumab, 293 patients with psoriasis were randomized to receive guselkumab, adalimumab, or placebo for 52 weeks. ${ }^{28}$ Patients received guselkumab $5 \mathrm{mg}$ at weeks 0 and 4 and every 12 weeks after; $15 \mathrm{mg}$ every 8 weeks; $50 \mathrm{mg}$ at weeks 0 and 4 and every 12 weeks after; $100 \mathrm{mg}$ every 8 weeks; or $200 \mathrm{mg}$ at weeks 0 and 4 and every 12 weeks after. The primary endpoint was a "clear/almost clear" score on the PGA at week 16. After 16 weeks, $34 \%$ in the $5-\mathrm{mg}$ guselkumab group, $61 \%$ in the 15 -mg group, $79 \%$ in the 50 -mg group, $86 \%$ in the 100 -mg group, and $83 \%$ in the 200 -mg group met this criterion compared with $7 \%$ of patients receiving placebo and $58 \%$ in the adalimumab group. At week $40,71 \%$ of the $50-\mathrm{mg}$ group, $77 \%$ of the $100-\mathrm{mg}$ group, and $81 \%$ of the $200-\mathrm{mg}$ group receiving guselkumab achieved a "clear/almost clear" score on the PGA compared with $49 \%$ of the adalimumab group.

The safety and efficacy of tildrakizumab were evaluated in a phase IIb trial in 355 patients with chronic plaque psoriasis. ${ }^{29}$ Patients received placebo or 5, 25, 100, or $200 \mathrm{mg}$ of tildrakizumab at weeks 0 and 4 and every 12 weeks after until
52 weeks. Treatment was discontinued at week 52, and patients were followed for 72 weeks. At week 16, PASI 75 responses were $33.3 \%$ in the 5 -mg group, $64.4 \%$ in the $25-\mathrm{mg}$ group, $66.3 \%$ in the $100-\mathrm{mg}$ group, and $74.4 \%$ in the $200-\mathrm{mg}$ group, compared with $4.4 \%$ in the placebo group. The PASI 75 response was maintained through 52 weeks, with 8 of the 222 participants who achieved PASI 75 response at week 52 experiencing a relapse after the discontinuation of the drug.

Two phase III studies investigated the efficacy and safety of oral tofacitinib compared with placebo in patients with moderate to severe chronic plaque psoriasis. ${ }^{30}$ The phase III studies (Oral treatment psoriasis trial (OPT) Pivotal 1 and 2) included 901 and 960 patients. Patients were administered 5 $\mathrm{mg}$ or $10 \mathrm{mg}$ of tofacitinib or placebo twice daily. At 16 weeks patients were evaluated for "clear/almost clear" response on PGA and a PASI 75 response. In OPT Pivotal 1, 41.9\% of the 5 -mg group and $59.2 \%$ of the $10-\mathrm{mg}$ group receiving tofacitinib achieved a PGA score of "clear/almost clear" at the end of 16 weeks compared with $9 \%$ in the placebo group. In OPT Pivotal 2, $46 \%$ of the 5-mg group and $59.1 \%$ of the $10-\mathrm{mg}$ group receiving tofacitinib achieved this score at the end of 16 weeks compared with $10.9 \%$ in the placebo group. In OPT Pivotal 1,39.9\% of the 5-mg group and 59.2\% of the $10 \mathrm{mg}$ group receiving tofacitinib achieved a PASI 75 response at week 16 compared with $6.2 \%$ of the placebo group. In OPT Pivotal 2, the PASI 75 response rates were $46 \%$ for the $5-\mathrm{mg}$ group and $59.6 \%$ for the $10 \mathrm{mg}$-group receiving tofacitinib compared with $11.4 \%$ of the placebo group.

A phase IIa dose-escalation RCT evaluated the efficacy and safety of ASP015K, another JAK inhibitor, in patients with moderate to severe psoriasis. ${ }^{31}$ The study enrolled 124 patients and had 5 sequential cohorts, 4 of which received $10,25,60$, or $100 \mathrm{mg}$ of ASP $015 \mathrm{~K}$ twice daily and one that received $50 \mathrm{mg}$ of the drug once daily. At the end of 6 weeks, the mean change in PASI score from baseline was measured (primary endpoint). APS015K demonstrated dose-dependent improvements in PASI scores over 6 weeks and was well tolerated with no serious adverse events reported.

An RCT of the anti-IL-23A monoclonal antibody BI 655066 for moderate to severe psoriasis in 39 patients found that the drug was well tolerated and was associated with clinical improvements in psoriasis. ${ }^{32}$ After 12 weeks of treatment, PASI 75, PASI 90, and PASI 100 were achieved by $87 \%, 58 \%$ and $16 \%$ of patients treated with the drug.

Refer to Figure 1 for the mechanisms of action of guselkumab, tildrakizumab, secukinumab, ixekizumab, brodalumab, and JAK inhibitors.

\section{Combination Therapy}

An analysis was performed to evaluate the effect of treatment with etanercept and topical corticosteroid therapy on HRQoL in psoriasis patients who were enrolled in the randomized, blinded assessor study to evaluate the efficacy and safety of etanercept $50 \mathrm{mg}$ once weekly plus as needed topical agent vs. etanercept $50 \mathrm{mg}$ twice weekly in patients with moderate 
to severe plaque psoriasis (REFINE) study. ${ }^{33}$ The REFINE study investigated the efficacy and safety of adding topical corticosteroid therapy to etanercept when stepping down from the initial dosage to the maintenance dosage of etanercept. In the REFINE study, 287 patients were randomized to receive etanercept $50 \mathrm{mg}$ twice weekly and then randomized at 12 weeks to either continue with twice-weekly etanercept or once-weekly doses plus topical corticosteroid as required until week 24. Topical agents included in the study were hydrocortisone $2.5 \%$, betamethasone valerate $0.1 \%$, betamethasone dipropionate $0.05 \%$, clobetasol $0.05 \%$, calcitriol, or calcipotriol plus betamethasone dipropionate $0.05 \%$. The HRQoL measures used in the analysis of these REFINE patients included the DLQI, the Treatment Satisfaction Questionnaire for Medication (TSqM), and the Economic Implications of Psoriasis Patient Questionnaire. The mean change recorded in standard deviation from baseline to week 24 for DLQI was 10.7 for etanercept and 9.9 for etanercept plus topical therapy. For the TSqM, the mean change (standard deviation) in effectiveness, convenience, side effects, and global satisfaction were 27.1, 14.8, -0.7 , and 26.7 for the etanercept group and $32.5,18.5,1.3$, and 28.4 for the etanercept plus topical therapy group, respectively. The economic implications were similar across both groups. At week 24, HRQoL measures were similar across both groups.

\section{References}

1. Kimball AB, Rothman KJ, Kriocorian G, et al. OBSERVE-5 observational postmarketing safety surveillance registry of etanercept for the treatment of psoriasis final 5-year results. J Am Acad Dermatol. 2015;72(1):115-122.

2. Menting SP, van Lumig PP, de Vries AC, et al. Extent and consequences of antibody formation against adalimumab in patients with psoriasis: one-year follow-up. JAMA Dermatol. 2014;150(2):130-136.

3. Koo J, Tyring S, Werschler WP, et al. Superior efficacy of calcipotriene and betamethasone dipropionate aerosol foam versus ointment in patients with psoriasis vulgaris - a randomized phase II study. J Dermatolog Treat. 2016;27(2):120-127.

4. Taraska V, Tuppal R, Olesen M, et al. A novel aerosol foam formulation of calcipotriol and betamethasone has no impact on HPA axis ad calcium homeostasis in patients with extensive psoriasis vulgaris. J Cutan Med Surg. 2016;20(1):44-51.

5. Landells I, Marano C, Hsu MC, et al. Ustekinumab in adolescent patients age 12 to 17 years with moderate-to-severe plaque psoriasis: results of the randomized phase 3 CADMUS study. J Am Acad Dermatol. 2015;73(4):594-603.

6. Gooderham M, Debarre JM, Keddy-Grant J, et al. Safety and efficacy of calcipotriol plus betamethasone dipropionate gel in the treatment of scalp psoriasis in adolescents 12-17 years of age. Br J Dermatol. 2014;171(6):1470-1477.

7. Oostveen AM, deJong EM, Donders AR, et al. Treatment of paediatric scalp psoriasis with calcipotriene/betamethasone dipropionate scalp formulation: effectiveness, safety and influence on children's quality of life in daily practice. J Eur Acad Dermatol Venereol. 2015;29(6):1193-1197.

8. Van Geel MJ, Mul K, Ooostven AM, et al. Calcipotriol/betamethasone dipropionate ointment in mild-to-moderate paediatric psoriasis: long-term daily clinical practice data in a prospective cohort. Br J Dermatol. 2014;171(2):363-369.

9. Bangsgaard N, Rorbye C, Skov L. Treating psoriasis during pregnancy: safety and efficacy of treatments. Am J Clin Dermatol. 2015;16(5):389-398.

10. Sanz-Bueno J, Vanaclocha F, Garcia-Doval I, et al. Risk of reactivation of hepatitis $B$ virus infection in psoriasis patients treated with biologics: a retrospective analysis of 20 cases from the BIOBADADERM Database. Actas Dermosifiliogr. 2015;106(6):477-482.

11. Omland SH, Gniadecki R. Psoriasis inversa: a separate identity or a variant of psoriasis vulgaris? Clin Dermatol. 2015;33(4):456-461.

12. Crowley JJ, Weinberg JM, Wu JJ, et al. Treatment of nail psoriasis: best practice recommendations from the Medical Board of the National Psoriasis Foundation. JAMA Dermatol. 2015;151(1):87-94.

13. Schons KRR, Beber AAC, Beck MO, et al. Nail involvement in adult patients with plaque-type psoriasis: prevalence and clinical features. An Bras Dermatol. 2015;90(3):314-319.

14. Rich P, Gooderham M, Bachelez H, et al. Apremilast, an oral phosphodiesterase 4 inhibitor, in patients with difficult-totreat nail and scalp psoriasis: results of 2 phase III randomized, controlled trials (ESTEEM 1 and ESTEEM 2). J Am Acad Dermatol. 2016;74(1):134-142.

15. Langley RG, Rich P, Menter A, et al. Improvement of scalp and nail lesions with ixekizumab in a phase 2 trial in patients with chronic plaque psoriasis. J Eur Acad Dermatol Venereol. 2015;29(9):1763-1770.

16. Reich K, Zschocke I, Bachelez H, et al. Efficacy of a fixed combination of calcipotriol/betamethasone dipropionate topical gel in adult patients with mild to moderate psoriasis: blinded interim analysis of a phase IV, multicenter, randomized, controlled, prospective study. J Eur Acad Dermatol Venereol. 2015;29(6):1156-1163.

17. Lambert J, Hol CW, Vink J. Real-life effectiveness of oncedaily calcipotriol and betamethasone dipropionate gel vs. ointment formulations in psoriasis vulgaris: final analysis of the 52-week PRO-long study. J Eur Acad Dermatol Venereol. 2015;29(12):2349-2355.

18. Lozinski A, Barzilai A, Pavlotsky F, et al. Broadband UVB versus paint PUVA for palmoplantar psoriasis treatment. J Dermatolog Treat. 2016;27(3):221-223.

19. Wald JM, Klufas DM, Strober BE. The use of methotrexate, alone or in combination with other therapies, for the treatment of palmoplantar psoriasis. J Drugs Dermatol. 2015;14(8):888-892.

20. Fleming P, Roubille C, Richer V, et al. Effect of biologics on depressive symptoms in patients with psoriasis: a systematic review. J Eur Acad Dermatol Venereol. 2015;29(6):1063-1070.

21. Ryan C, Sadlier M, De Vol E, et al. Genital psoriasis is associated with significant impairment in quality of life and sexual functioning. J Am Acad Dermatol. 2015;72(6):978-983.

22. Parisi R, Rutter MK, Lunt M, et al. Psoriasis and the risk of major cardiovascular events: cohort study using the Clinical Practice Research Datalink. J Invest Dermatol. 2015;135(9):2189-2197.

23. Chiu HY, Huang HL, Li CH, et al. Increased risk of glomerulonephritis and chronic kidney disease in relation to the severity of psoriasis, concomitant medication, and comorbidity: a nationwide population-based cohort study. $\mathrm{Br} J$ Dermatol. 2015;173(1):146-154. 
24. Lebwohl M, Strober B, Menter A, et al. Phase 3 studies comparing brodalumab with ustekinumab in psoriasis. $N$ Engl $J$ Med. 2015;373(14):1318-1328.

25. Gordon KB, Leonardi CL, Lebwohl M, et al. A 52-week, open-label study of the efficacy and safety of ixekizumab, an anti-interleukin-17A monoclonal antibody, in patients with chronic plaque psoriasis. $J$ Am Acad Dermatol. 2014;71(6):1176-1182.

26. Griffiths CE, Reich K, Lebwohl M, et al. Comparison of ixekizumab with etanercept or placebo in moderate-to-severe psoriasis (UNCOVER-2 and UNCOVER-3): results from two phase 3 randomised trials. Lancet. 2015;386(9993):541-551.

27. Langley RG, Elewski BE, Lebwohl M, et al. Secukinumab in plaque psoriasis - results of two phase 3 trials. $N$ Engl J Med. 2014;371(4):326-338.

28. Gordon KN, Callis Duffin K, Bissonnette R, et al. A phase 2 trial of guselkumab versus adalimumab for plaque psoriasis. N Engl J Med. 2015;373:136-144.

29. Papp K, Thaci D, Reich K, et al. Tildrakizumab (MK-3222), an anti-interleukin-23p19 monoclonal antibody, improves psoriasis in a phase IIb randomized placebo-controlled trial. Br J Dermatol. 2015;173(4):930-939.

30. Papp KA, Menter MA, Abe M, et al. Tofacitinib, an oral Janus kinas inhibitor, for the treatment of chronic plaque psoriasis: results from two randomized, placebo-controlled, phase III trials. Br J Dermatol. 2015;173(4):949-961.

31. Papp K, Pariser D, Catlin M, et al. A phase 2a randomized, double-blind, placebo-controlled, sequential dose-escalation study to evaluate the efficacy and safety of ASP015K, a novel Janus kinase inhibitor, in patients with moderate-to-severe psoriasis. Br J Dermatol. 2015;173(3):767-776.

32. Krueger JG, Ferris LK, Menter A, et al. Anti-IL-23A mAb BI 655066 for treatment of moderate-to-severe psoriasis: safety, efficacy, pharmacokinetics, and biomarker results of a singlerising-dose, randomized, double-blind, placebo-controlled trial. J Allergy Clin Immunol. 2015;136(1):116-124.

33. Papp KA, Barber K, Bissonnette R, et al. Improvements in patient-reported outcomes in patients with psoriasis receiving etanercept plus topical therapies: results from REFINE. J Eur Acad Dermatol Venereol. 2015:29(8):1555-1561. 


\section{Appendix I: Trade Name/Generic Name Translator}

Because generic names are used throughout these Guidelines, text searches of the electronic document using trade names for drugs will not identify the relevant pages. Readers who are uncertain of the correct generic name of a drug may consult the table below to identify a searchable drug name.

Although this list is thorough, it is provided as a guide only; it may not be complete.

\begin{tabular}{|c|c|c|}
\hline Trade Name or Trivial Name & Generic Name & Notes \\
\hline Amevive & Alefacept & Removed from Canadian market in $201 \mathrm{I}$ \\
\hline Anthraforte & Anthralin & \\
\hline Anthranol & Anthralin & \\
\hline AnthraScalp & Anthralin & \\
\hline Apo-Cyclosporine & Cyclosporine & \\
\hline Apo-Hydroxyquine & Hydroxychloroquine sulfate & \\
\hline Apo-Lithium Carbonate & Lithium & Formulated as lithium carbonate \\
\hline Apo-Methotrexate & Methotrexate & \\
\hline Apo-Simvastatin & Simvastatin & \\
\hline Arava & Leflunomide & \\
\hline Aristocort & Triamcinolone & Formulated as triamcinolone acetonide \\
\hline Avastin & Bevacizumab & \\
\hline Betaderm & Betamethasone & Formulated as betamethasone valerate \\
\hline Betaject & Betamethasone & $\begin{array}{l}\text { Formulated as betamethasone } 21 \text {-disodium phosphate plus } \\
\text { betamethasone acetate }\end{array}$ \\
\hline Betnesol & Betamethasone & Formulated as betamethasone sodium phosphate \\
\hline Carbolith & Lithium & Formulated as lithium carbonate \\
\hline $\begin{array}{l}\text { Celestone Soluspan } \\
\text { Injectable }\end{array}$ & Betamethasone & $\begin{array}{l}\text { Formulated as betamethasone sodium phosphate plus betamethasone } \\
\text { acetate }\end{array}$ \\
\hline Cimzia & Certolizumab & \\
\hline Clobex & Clobetasol & \\
\hline Cosentyx & Secukinumab & \\
\hline Cutivate & Fluticasone propionate & \\
\hline Dermovate & Clobetasol & \\
\hline Diprolene Glycol & Betamethasone & Formulated as betamethasone dipropionate \\
\hline Diprosalic & Betamethasone & Formulated as betamethasone dipropionate plus salicylic acid \\
\hline Diprosone & Betamethasone & Formulated as betamethasone dipropionate \\
\hline Dovobet & Calcipotriol/betamethasone & $\begin{array}{l}\text { Formulated as calcipotriol/betamethasone dipropionate gel and foam. } \\
\text { The ointment formulation has been discontinued. }\end{array}$ \\
\hline Dovonex & Calcipotriol & \\
\hline Duralith & Lithium & Formulated as lithium carbonate \\
\hline Denorex & Coal tar & Formulated as coal tar plus chloroxylenol and menthol \\
\hline Dithranol & Anthralin & \\
\hline Doak Oil & Coal tar & \\
\hline Elidel & Pimecrolimus & \\
\hline Enbrel & Etanercept & \\
\hline Exorex & Coal tar & \\
\hline Gen-Clobetasol & Clobetasol & \\
\hline Gen-Hydroxychloroquine & Hydroxychloroquine sulfate & \\
\hline Humira & Adalimumab & \\
\hline Jakavi & Ruxolitinib & \\
\hline
\end{tabular}


Appendix I. (continued)

\begin{tabular}{|c|c|c|}
\hline Trade Name or Trivial Name & Generic Name & Notes \\
\hline Lidemol & Fluocinonide & \\
\hline Lidex & Fluocinonide & \\
\hline Lithane & Lithium & Formulated as lithium carbonate \\
\hline Lithium Benziocum & Lithium & Formulated as lithium benzoate \\
\hline Lithium Carbonicum & Lithium & Formulated as lithium carbonate \\
\hline Locasalen & Flumethasone & \\
\hline Lyderm & Fluocinonide & \\
\hline Mazon Medicated Cream & Coal tar & Formulated as coal tar plus resorcinol and salicylic acid \\
\hline Medi-Dan Shampoo & Coal tar & Formulated as coal tar plus benzalkonium chloride and salicylic acid \\
\hline Metoject & Methotrexate & \\
\hline Multi-Tar Plus Shampoo & Coal tar & Formulated as coal tar plus juniper tar, pine tar, and pyrithione zinc \\
\hline Neoral & Cyclosporine & \\
\hline Nexavar & Sorafenib & \\
\hline Novo-Chloroquine & Chloroquine & \\
\hline Novo-Clobetasol & Clobetasol & \\
\hline $\begin{array}{l}\text { Odans Liquor Carbonis } \\
\text { Detergens }\end{array}$ & Coal tar & Formulated as coal tar plus benzocaine and salicylic acid \\
\hline Otezla & Apremilast & \\
\hline Oxsoralen & Methoxsalen & \\
\hline P\&S Plus & Coal tar & Formulated as coal tar plus salicylic acid \\
\hline PHL-Lithium Carbonate & Lithium & \\
\hline Plaquenil & Hydroxychloroquine sulfate & \\
\hline PMS-Lithium Carbonate & Lithium & Formulated as lithium carbonate \\
\hline Prevex B & Betamethasone & Formulated as betamethasone valerate \\
\hline Pro-Hydroxyquine & Hydroxychloroquine sulfate & \\
\hline Protopic & Tacrolimus & \\
\hline Psoriasin & Coal tar & \\
\hline Raptiva & Efalizumab & Removed from Canadian market in 2009 \\
\hline Ratio-Clobetasol & Clobetasol & \\
\hline Ratio-Ectosone & Betamethasone & \\
\hline Ratio-Fluticasone & Fluticasone propionate & \\
\hline Ratio-Methotrexate Sodium & Methotrexate & Formulated as betamethasone valerate \\
\hline Ratio-Topilene & Betamethasone & Formulated as betamethasone dipropionate \\
\hline Ratio-Topisalic & Betamethasone & Formulated as betamethasone dipropionate plus salicylic acid \\
\hline Rivasone Scalp & Betamethasone & Formulated as betamethasone valerate \\
\hline Rolene & Betamethasone & Formulated as betamethasone dipropionate \\
\hline Rosone & Betamethasone & Formulated as betamethasone dipropionate \\
\hline Sandoz Cyclosporine & Cyclosporine & \\
\hline SJ Liniment & Coal tar & $\begin{array}{l}\text { Formulated as coal tar plus ammonium hydroxide, menthol, and } \\
\text { methyl salicylate }\end{array}$ \\
\hline Sebcur & Coal tar & Formulated as coal tar plus salicylic acid \\
\hline Silkis & Calcitriol & \\
\hline Simponi & Golimumab & \\
\hline Stelara & Ustekinumab & \\
\hline Sterex & Coal tar & Formulated as coal tar plus salicylic acid, sulfur, and/or hydrocortisone \\
\hline Sutent & Sunitinib malate & \\
\hline Soriatane & Acitretin & \\
\hline $\begin{array}{l}\text { T Gel; T/Gel Therapeutic } \\
\text { Shampoo }\end{array}$ & Coal tar & \\
\hline Tardan & Coal tar & Formulated as coal tar plus salicylic acid and triclosan \\
\hline Targel & Coal tar & \\
\hline Taro-Clobetasol & Clobetasol & \\
\hline Taro-Sone & Betamethasone & Formulated as betamethasone dipropionate \\
\hline
\end{tabular}


Appendix I. (continued)

\begin{tabular}{lll}
\hline Trade Name or Trivial Name & \multicolumn{1}{c}{ Generic Name } & Notes \\
\hline Tazorac & Tazarotene & \\
Tersa Tar Shampoo & Coal tar & \\
Tiamol & Fluocinonide & \\
Topactin & Fluocinonide & \\
Topsyn & Fluocinonide & \\
Ultravate & Halobetasol propionate & Formulated as coal tar plus menthol and salicylic acid \\
X-Seb T-plus Conditioning & Coal tar & \\
Xamiol & Calcipotriol/betamethasone & Removed from Canadian market in 20I2 \\
Xeljanz & Tofacitinib & \\
Zocor & Simvastatin & \\
\hline
\end{tabular}




\section{Appendix II: Clinical Questions to Guide Addendum on Management Recommendations (Listed by Chapter)}

\section{Chapter 5: Management of Mild Plaque Psoriasis}

1) What treatments should be considered first-line for mild plaque psoriasis?

2) What treatments should be considered second-line for this indication?

3) What treatments should be considered third-line for this indication?

4) When should combination therapy be considered?

5) What combination therapy should be considered for this indication?

6) When should nonmedicinal agents be considered?

7) What set of criteria are used to judge successful management of mild plaque psoriasis?

\section{Chapter 6: Management of Moderate to Severe Plaque Psoriasis}

1) What treatments should be considered first-line for moderate to severe plaque psoriasis?

2) What treatments should be considered second-line for this indication?

3) What treatments should be considered third-line for this indication?

4) When should combination therapy be considered?

5) What combination therapy should be considered for this indication?

6) What set of criteria are used to judge successful management of moderate to severe plaque psoriasis?

\section{Chapter 7: Special Populations and Circumstances}

1) What populations or circumstances warrant special consideration for disease management?

2) What treatments should be considered for each special population or circumstance?

3) What set of criteria should be used to judge successful management of plaque psoriasis in each special population or circumstance?

4) What are the treatment considerations for the use of biologics in pregnancy?

5) What triggers are there for adding a concomitant therapy?

6) What triggers exist for withdrawing therapy?
7) When is it appropriate for drug holidays or rotational therapy?

\section{Chapter 8: Exacerbation and Flare of Psoriasis}

1) What criteria are used to identify an exacerbation?

2) What criteria are used to identify a disease flare?

3) What criteria are used to identify disease rebound?

4) What triggers lead to exacerbations?

5) What triggers lead to flares?

6) When is it appropriate for drug holidays or rotational therapy?

\section{Chapter 9: Management of Facial, Flexural, and Genital Psoriasis}

1) What set of criteria are used to judge successful management of facial, flexural, and genital psoriasis?

2) What treatments should be considered first-line for this indication?

3) What treatments should be considered second-line for moderate disease?

4) What treatments should be considered third-line for moderate to severe disease?

5) What triggers are there for adding a concomitant therapy?

6) What triggers exist for withdrawing therapy?

7) When is it appropriate for drug holidays or rotational therapy?

\section{Chapter 10: Management of Nail Psoriasis}

1) What criteria are used to evaluate mild, moderate, or severe nail psoriasis?

2) What are appropriate clinical goals for the treatment of nail psoriasis?

3) What treatments should be considered first-line in managing nail psoriasis?

4) What are second-line treatments for nail psoriasis?

5) What treatments are effective for hyperkeratosis?

6) How should isolated nail psoriasis be treated?

7) What are appropriate treatments for patients with mild, moderate, or severe psoriasis with nail involvement? 


\section{Chapter I I: Management of Scalp Psoriasis}

1) How should mild, moderate, or severe psoriasis be assessed in patients with scalp psoriasis?

2) What are appropriate clinical goals for the treatment of symptoms of scalp psoriasis?

3) What treatments should be considered first-line in managing scalp psoriasis?

4) What second-line approaches to scalp psoriasis are appropriate for patients who fail to respond to topical treatments or home UV therapy?

5) What pretreatments are effective for achieving keratolysis in patients with scalp psoriasis?

6) What are second-line options for treating recalcitrant scalp psoriasis?

\section{Chapter 12: Management of Palmoplantar Psoriasis}

1) How should disease severity be assessed in patients with plaque-type palmoplantar psoriasis?

2) How should severity of PPP be assessed?

3) What are appropriate clinical goals in the treatment of symptoms of palmoplantar psoriasis?

4) Should symptoms of PPP (plaque-type or pustular) be addressed independently of any treatments for plaque psoriasis?

5) How should recalcitrant plaque-type palmoplantar psoriasis be treated?

6) What are the first-line options for treatment of palmoplantar pustulosis?

7) What are the second-line, potentially remittive therapeutic options for treating palmoplantar pustulosis?

8) How should recalcitrant palmoplantar pustulosis be treated?

\section{Chapter 13: Social and Psychological Aspects of Psoriasis}

1) What are the social and psychological aspects of psoriasis?
2) How should the social and psychological impact of psoriasis be assessed?

3) How should social or psychological impacts of psoriasis be managed?

\section{Chapter 14: Comorbidities}

1) What are the comorbidities commonly associated with psoriasis?

2) What comorbidities should be screened for in patients with psoriasis?

3) What triggers exists for these comorbidities?

4) How should patients with comorbidities be managed?

5) When should patients with comorbidities be referred to a specialist?

\section{Chapter 15: The Future of Psoriasis Care}

1) Are any changes to standards of care for psoriasis anticipated in the near future?

2) What novel treatments are on the horizon for psoriasis management?

3) What role will biosimilars play in the treatment of psoriasis?

\section{Chapter 16: Combination Therapy for Plaque Psoriasis}

1) What are the considerations when choosing a combination therapy?

2) What criteria should be used to judge the success of a combination therapy?

3) When should combination therapy be modified or changed?

4) How should combined therapy be modified or changed?

\section{Chapter 17: Summary of New Findings}

1) What are the pertinent papers in the literature on the management of plaque psoriasis published from January 2014 to December 2015? 